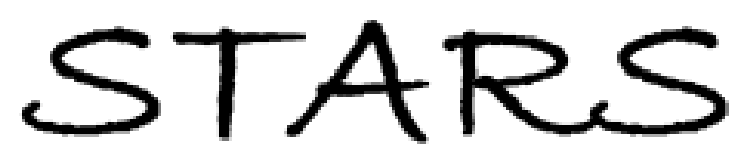

University of Central Florida

STARS

Retrospective Theses and Dissertations

1996

\title{
Analysis of Frequency Selective Surfaces with Ferrite Substrates
}

Youcheng Liu

University of Central Florida

Part of the Electrical and Computer Engineering Commons

Find similar works at: https://stars.library.ucf.edu/rtd

University of Central Florida Libraries http://library.ucf.edu

This Doctoral Dissertation (Open Access) is brought to you for free and open access by STARS. It has been accepted for inclusion in Retrospective Theses and Dissertations by an authorized administrator of STARS. For more information, please contact STARS@ucf.edu.

\section{STARS Citation}

Liu, Youcheng, "Analysis of Frequency Selective Surfaces with Ferrite Substrates" (1996). Retrospective Theses and Dissertations. 2996.

https://stars.library.ucf.edu/rtd/2996

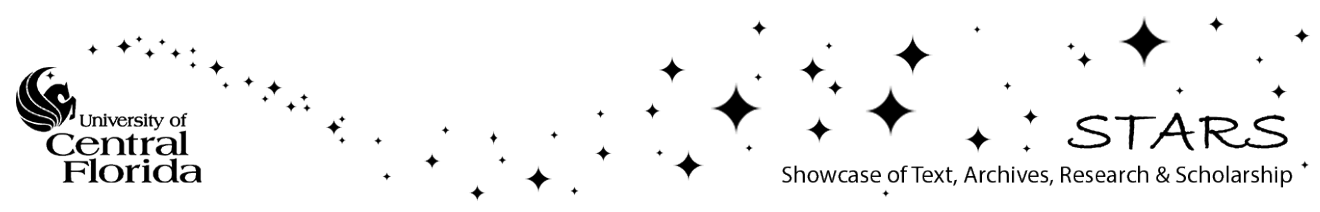




\title{
ANALYSIS OF FREQUENCY SELECTIVE SURFACES WITH FERRITE SUBSTRAIES
}

\author{
By \\ Yerdiserg Lit
}

1996

UCF

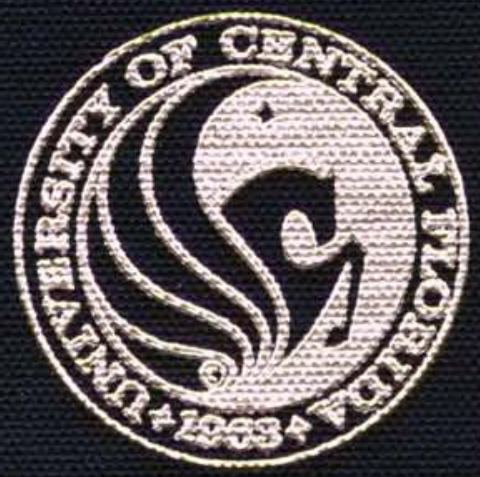




\title{
ANALYSIS OF FREQUENCY SELECTIVE SURFACES WITH FERRITE SUBSTRATES
}

\author{
by \\ YOUCHENG LIU
}

B.S.E.E., Shanghai Jiao-Tong University, 1982

M.S.E.E., Shanghai Jiao-Tong University, 1990

\section{DISSERTATION}

Submitted in partial fulfillment of the requirements for the degree of

Doctor of Philosophy

Department of Electrical and Computer Engineering

College of Engineering

University of Central Florida

Orlando, Florida

Spring Term

1996 


\section{ABSTRACT}

Frequency selective surfaces (FSS) have numerous applications in several microwave and optical systems. Most frequency selective surface structures have one or more dielectric substrates. In this work, instead of traditional dielectric substrates, ferrite substrates are used under different dc bias conditions. By using ferrite materials, one can change the spectral properties of these structures without physically altering them. An applied magnetic field (dc bias) on the ferrite substrate changes its properties and hence the electrical dimensions of the elements comprising the periodic structure. Thus by simply applying a dc bias, the transmission and reflection properties of the periodic structure can be changed. That leads to a tuning mechanism which allows the designer, by varying the externally applied dc magnetic field, to obtain a more desirable frequency response.

In this work, the transmission matrices for the ferrite substrate and the air, above and below the ferrite substrate are derived. By combining these transmission matrices along with the boundary conditions, the spectral domain Green's function is obtained. This process is carried out for both the in-plane bias and perpendicular bias of the ferrite. The induced current on the conductor patch is solved by the method of moments in the spectral domain. Roof toping functions are used as both expansion and test functions. 
Several results are presented to show the tunability of frequency selective surfaces with ferrite substrates as a function of the applied dc bias. Other unique characteristics of the frequency selective surfaces on ferrite substrates are also presented and discussed. The design procedure for frequency selective surfaces by neural network algorithms is introduced. 


\section{ACKNOWLEDGEMENTS}

I would like to take this opportunity to express the gratitude to my advisor, Dr. Christos G. Christodoulou, for guidance, discussion and all the support for my Ph.D. studies.

I would like to thank Dr. P. Wahid, Dr. M. Georgiopoulos, Dr. A. Motazawi, and Dr. $\mathrm{X} . \mathrm{Li}$, for their helpful discussion, suggestions and taking the time to be on my committee and reviewing the dissertation. I would also like to thank the colleagues at the EM group who provided assistance and suggestions.

Finally, I would like to thank my wife Jie for her support and patience, my son Xin for his understanding, and my other family members for their love and encouragement. 


\section{TABLE OF CONTENTS}

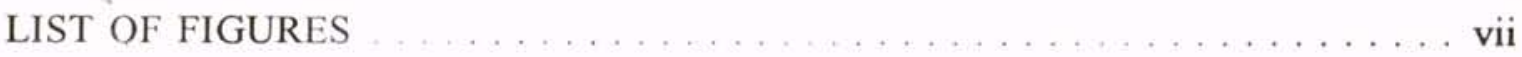

CHAPTER 1 - INTRODUCTION . . . . . . . . . . . . 1

CHAPTER 2 - FREE-STANDING FREQUENCY SELECTIVE SURFACES . . . . 5

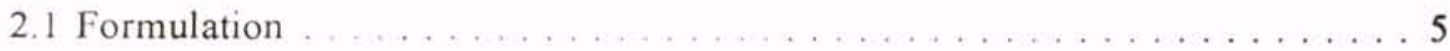

2.2 The Method of Moments . . . . . . . . . . . . . . . . 10

2.3 Basis Functions and Test Functions $\ldots \ldots \ldots \ldots \ldots \ldots \ldots \ldots \ldots$

2.4 Matrix Equation of the Scattered Field . . . . . . . . . . . . 17

2.5 Reflection Coefficients .......................... 19

CHAPTER 3 - PROPERTIES OF FERRITE MATERIALS . . . . . . . 22

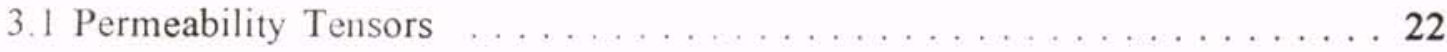

3.2 The Interaction of an AC Signal with a Biased Ferrite $\ldots \ldots \ldots \ldots$

3.3 Plane Wave Propagation in Ferrite Medium .............. 33

3.3 .1 Faraday Rotation . ........................ 35

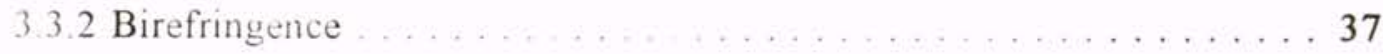

CHAPTER 4 - ANALYSIS OF FREQUENCY SELECTIVE SURFACES WITH FERRITE SUBSTRATES .............. 40

4.1 Derivation of Green's Function for Ferrite Substrates . . . . . . . . . 40

4.2 Power Reflection Coefficient ... . . . . . . . . . . . . . . . . . . . 59

CHAPTER 5 - NUMERICAL RESULTS . . . . . . . . . . . . 60

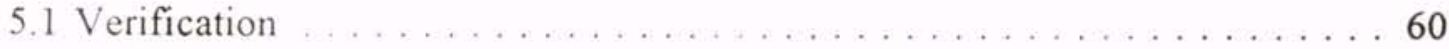

5.2 Results for FSS on Ferrite Substrates .................. 64

5.3 Discussion . . . . . . . . . . . . . . . . . . . . . 77 
CHAPTER 6 - DESIGN OF FREQUENCY SELECTIVE SURFACES USING NEURAL NETWORK ALGORITHMS . . . . . . . . . 87

CHAPTER 7 - FUTURE WORK . . . . . . . . . . . . . . . . . . 99

APPENDIX A - EXPRESSION OF GREEN'S FUNCTIONS FOR FSS ON FERRITE SUBSTRATES $\ldots \ldots \ldots \ldots \ldots \ldots \ldots \ldots$ APPENDIX B - ELEMENT EXPRESSIONS FOR Z-BIAS CASE . . . . . 103 APPENDIX C - DESCRIPTION OF NEURAL NETWORK ALGORITHMS USEDI05

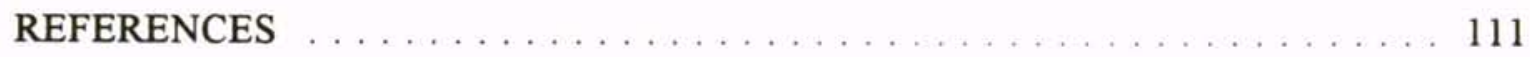

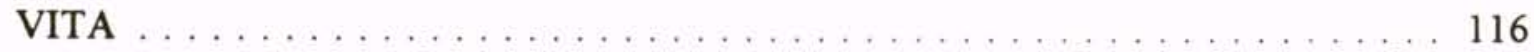




\section{LIST OF FIGURES}

1. Geometry of a free-standing FSS $\ldots \ldots \ldots \ldots \ldots \ldots \ldots \ldots \ldots \ldots \ldots \ldots \ldots$

2. Geometry of roof-toping subdomain basis function $\ldots \ldots \ldots \ldots \ldots$

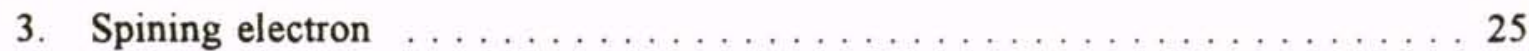

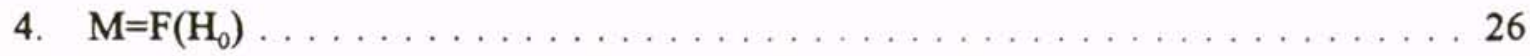

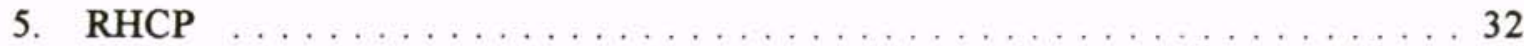

6. $\quad$ LHCP . . . . . . . . . . . . . . . . . . . . . . . . . . . 34

7. (a) Geometry of a FSS on ferrite substrate $\ldots \ldots \ldots \ldots \ldots \ldots \ldots \ldots \ldots \ldots$

(b) Sideview of a FSS on ferrite substrate $\ldots \ldots \ldots \ldots \ldots \ldots \ldots \ldots \ldots$

8. Geometry of a FSS unite cell (patch) . . . . . . . . . . . . . 62

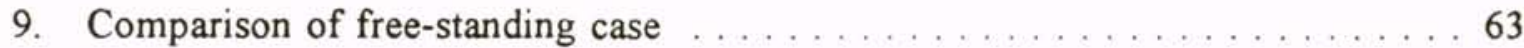

10. Frequency response with different $\mathrm{H}_{0}$ at $\mathrm{d}=0.02 \mathrm{~cm} \ldots \ldots \ldots 66 \ldots \ldots \ldots 6$

11. Frequency response with different $\mathrm{H}_{0}$ at $\mathrm{d}=0.04 \mathrm{~cm} \ldots \ldots \ldots 6 . \ldots \ldots 6$

12. Frequency response with different $\mathrm{H}_{0}$ at $\mathrm{d}=0.06 \mathrm{~cm} \ldots \ldots \ldots 68 \ldots \ldots 6 . \ldots 6$

13. Frequency response with different $\mathrm{H}_{0}$ at $\mathrm{d}=0.08 \mathrm{~cm} \ldots \ldots \ldots . \ldots 69$

14. Frequency response with different $\mathrm{H}_{0}$ at $\varepsilon_{\mathrm{r}}=15, \mathrm{~d}=0.02 \mathrm{~cm} \ldots \ldots \ldots \ldots 70$

15. Frequency response with different $\mathrm{H}_{0}$ at $\varepsilon_{\mathrm{r}}=15, \mathrm{~d}=0.08 \mathrm{~cm} \ldots \ldots \ldots \ldots 71$

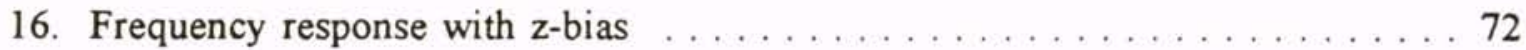

17. Frequency response with different thickness $d$ at $\mathrm{H}_{0}=20000$ e . . . . . . 73

18. Frequency response with different thickness $d$ at $\mathrm{H}_{0}=30000$ e . . . . . . . 74

19. Frequency response with different thickness $d$ at $\mathrm{H}_{0}=40000$ e . . . . . . . 75

20. Frequency response with different thickness $d$ at $\mathrm{H}_{0}=50000$ e . . . . . 76

21. Frequency response with different thickness $d$ at $\mathrm{H}_{0}=20000 \mathrm{e}, \varepsilon_{\mathrm{r}}=15 \ldots \ldots 78$ 
22. Frequency response with different thickness $\mathrm{d}$ at $\mathrm{H}_{0}=4000 \mathrm{Oe}, \varepsilon_{\mathrm{r}}=1579 \ldots 79$

23. Frequency response with different $\varepsilon_{r}$ at $\mathrm{H}_{0}=200000 \mathrm{e}, \mathrm{d}=0.02 \mathrm{~cm} \ldots \ldots 8$

24. Frequency response with different $\varepsilon_{\mathrm{r}}$ at $\mathrm{H}_{0}=40000 \mathrm{Oe}, \mathrm{d}=0.02 \mathrm{~cm} \ldots \ldots 1$

25. Frequency response with different $\varepsilon_{\mathrm{r}}$ at $\mathrm{H}_{0}=20000 \mathrm{Oe}, \mathrm{d}=0.08 \mathrm{~cm} \ldots . . .82$

26. Frequency response with different $\varepsilon_{\mathrm{r}}$ at $\mathrm{H}_{0}=40000 \mathrm{Oe}, \mathrm{d}=0.08 \mathrm{~cm} \ldots \ldots 3$

27. Comparison of different Floquet modes . . . . . . . . . . . . . . . . 84

28. The flow chart of back-propagation algorithm $\ldots \ldots \ldots \ldots \ldots \ldots$

29. The flow chart of neural network inversion algorithm . . . . . . . . . 92

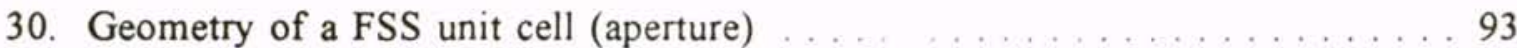

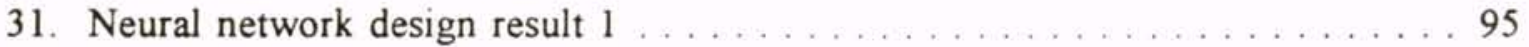

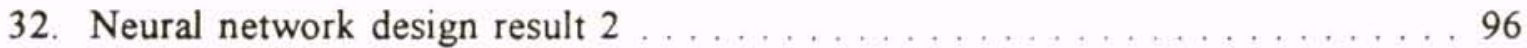

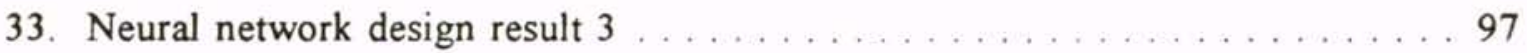

34. Neural network design result $4 \ldots \ldots \ldots \ldots \ldots$ 


\section{CHAPTER 1}

\section{INTRODUCTION}

Frequency selective surfaces (FSS) have wide applications over much of the electromagnetic spectrum. They are mainly used as reflector antenna dichroic surfaces, microwave filters or antenna passband radomes in the microwave region. They are also used as beam splitters, mirrors for laser applications. Frequency selective surfaces are basically composed of periodically arranged metallic patch elements or periodic arrays of apertures. They exhibit total reflection or transmission in the neighborhood of the element resonance.

The principle of frequency selective surface, as an investigation of diffraction gratings in optics, was first discovered by the American physicist David Rittenhouse in 1786 [1]. Because of the difficulties of computation, the first application of his discovery as a frequency selective surface appeared late in the 1960's. The analysis of frequency selective surfaces was first carried out by using the conventional mode-matching technique [2], [3]. The so called mode-matching technique makes use of the Floquet modes describing the periodic structure, setting up field equations in time domain. By using the appropriate boundary conditions, the scattered fields are related to the incident fields. This leads to an integral equation, which is then solved by the method of moments. The advantage of using mode-matching is that the resultant expressions are physically 
explicit. In the 1980's, the Spectral Domain Method, associated with the Fast Fourier Transform (FFT) algorithm was developed [4]-[8]. By applying the Fourier transform, the convolution form of the integral equation for the induced current is reduced to an algebraic one in the spectral domain. The analysis of frequency selective surfaces becomes more computationally efficient by using this approach.

There are a variety of approaches that can be used with the Spectral Domain Method. The most conventional and widely used approach is the Method of Moments (MM) [3], [7]-[10]. The concept of the Method of Moments is to assume that the unknown induced current on the metallic patches can be expanded in terms of known basis functions such that the integral equation is transformed into a matrix equation. The inversion of a matrix is required for this method. Another commonly used approach is the Conjugate Gradient Method (CGM) [11]-[15] and [8]. Usually, the Conjugate Gradient Method is computationally simpler than the method of moments. Theoretically, the approximate solution will converge to the exact solution in a finite number of steps starting with any initial value if the system matrix is positive definite [13].

At first, the analysis of frequency selective surfaces was restricted to the freestanding case [2], [3], [7], [8], [10]-[12], [14]-[18]. Then, frequency selective surfaces with dielectric substrate were considered [19]-[25]. Later on, the generalized analysis method for multilayer cascaded connection surfaces was presented [26]-[29]. C. K. Lee, and R. J. Langley used equivalent-circuit models to simulate frequency selective surfaces with oblique incident angles [30]. R. J. Rubin, and H. L. Bertoni approximated induced current on the patches with roof-top subdomain basis functions [10]. C. H. Chan used 
the Fast Fourier Transform algorithm to calculate frequency selective surfaces efficiently [31]. T. K. Wu developed and tested a single-screen frequency selective surface for triband applications [32]. W. L. Ko, and R. Mittra extended the method of analysis developed for the planar-array case to the problem of a conformal array arranged on a parabolic cylindrical surface with the objective of computing the scattering properties of the curved array [33]. P. W. Grounds and K. J. Webb presented the results of an analysis of a finite frequency selective surface instead of conventional infinite frequency selective surfaces [34]. R. Mittra, C. H. Chan and T. Cwik in their " Techniques for Analyzing Frequency Selective Surfaces - A Review " [1] systematically summarized a number of typical techniques.

Due to the recent progress in the growth and deposition of magnetic films on semiconductors, there is a renewed interest in the characteristics of microwave structures on ferrite substrates [35]-[38]. Y. Liu, et al in [39], G. Y. Li, et al in [40] presented the analysis results of frequency selective surfaces on ferrite substrate, simultaneously. T. K. Chang, R. J. Langley, and E. A. Parker discussed the influence of biased ferrite substrates upon the frequency characteristics of frequency selective surfaces by their experiments [41].

Ferrite materials offer a unique design capability due to their gyrotropic nature. They have a permeability tensor whose elements can be easily controlled through the use of a dc magnetic bias field. The extra degree of freedom offered by the biased ferrite substrates can be used to obtain a number of novel characteristics. In this dissertation, a systematic analysis of frequency selective surfaces on ferrite substrates is presented. 
The spectral domain Green's functions for both in-plane bias and perpendicular bias cases are derived. Several calculated results are presented to show the tunability of frequency selective surfaces with ferrite substrates. Other unique characteristics of the frequency selective surfaces on ferrite substrates are also discussed. To verify these models, calculated results from this model are compared with the published ones for free-standing and frequency selective surfaces on dielectric substrates.

Chapter 2 of the dissertation gives a general description of the Spectral Domain analysis of free-standing frequency selective surfaces. The roof toping subdomain functions are introduced. With the model of free-standing analysis, the model of frequency selective surfaces with ferrite substrates is then easily derived. In Chapter 3, the gyrotropic properties of ferrite material are discussed, including the derivation of the permeability tensors, and the different modes of microwave propagation in a ferrite medium. Chapter 4 gives the detailed derivation of the Green's functions for the frequency selective surfaces on ferrite substrates with both in-plane and perpendicular bias. Chapter 5 shows the comparison of the results calculated with the model developed in this dissertation to the data from the published papers. Several calculated results are presented to demonstrate the tunability of the frequency selective surfaces on ferrite substrates as a function of the dc bias field. Other properties of the frequency selective surfaces on ferrite substrates are also discussed. In Chapter 6, a frequency selective surface design procedure with neural network technique is presented. Chapter 7 includes recommendations for future work. 


\section{CHAPTER 2}

\section{FREE-STANDING FREQUENCY SELECTIVE SURFACES}

\subsection{FORMULATION}

A free-standing frequency selective surface is the simplest case of frequency selective surfaces analysis. Some assumptions are made prior to the analysis and they are held true for the entire work in this dissertation. First, the frequency selective surface is infinite in extent. Second, the metallic patch is infinitesimally thin, therefore, the induced current density on the patch has only transverse components. Third, the patch is a perfect conductor. The third assumption is made only for convenience. It can be easily modified to be used for a patch with finite conductivity.

The configuration of a free-standing frequency selective surface is shown in Figure 1. The patches are extended infinitely in both $\mathrm{x}$ and $\mathrm{y}$ directions. The periodicities are "a" and "b" in $\mathrm{x}$ and $\mathrm{y}$ directions, respectively.

When a plane wave excites a periodic structure surface, the induced current density on the patches as well as the associated scattered fields are all periodic functions with an exponential phase factor [44]. The analysis of the frequency selective surface can be done for a unit cell. The periodic nature of the current and fields also allows the use of Fourier expansion to relate the scattered fields to the surface current density. This mean 


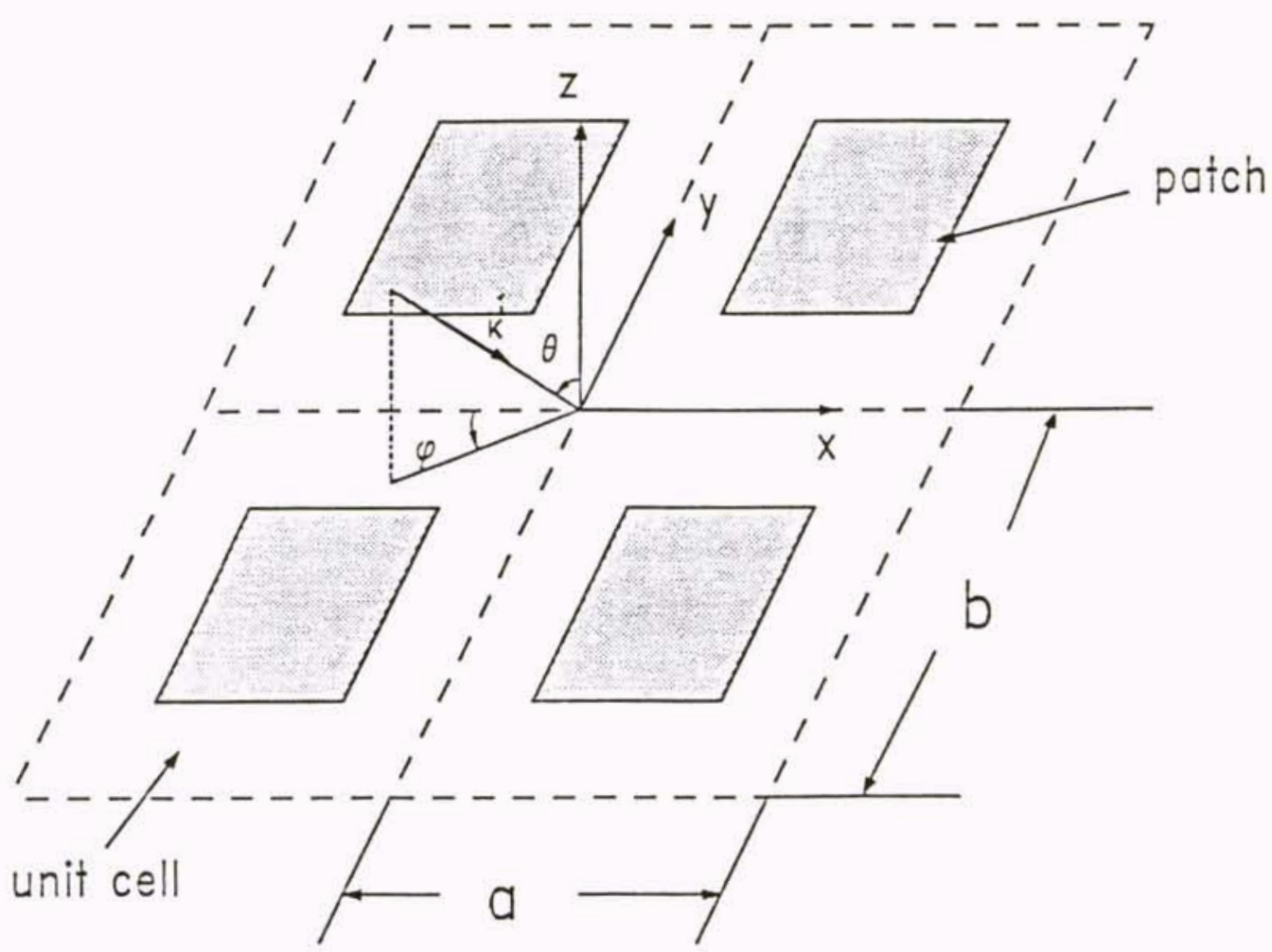

Figure 1. Geometry of a free-standing frequency selective surface. 
that if the analysis is performed in the spectral domain it will dramatically simplify the operation.

Let $\mathbf{A}$ be the magnetic vector potential due to the induced current density $\mathbf{J}$ on the patch. Since $\mathbf{J}$ has only transverse components, the magnetic vector potential $\mathbf{A}$ will also

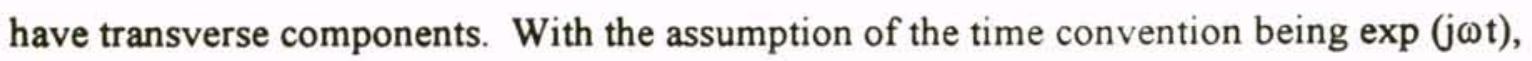
the relation between $\mathbf{A}$ and $\mathbf{J}$ has the following form:

$$
\boldsymbol{A}(x, y)=\boldsymbol{G}(x, y) *\left[\begin{array}{l}
J_{x}(x, y) \\
J_{y}(x, y)
\end{array}\right]
$$

where $" * "$ is the convolution operator,

$$
\boldsymbol{G}(x, y)=\frac{\exp \left(-j k_{0} I\right)}{4 \pi I} I
$$

with

$$
k_{0}=\omega \sqrt{\mu_{0} \epsilon_{0}}
$$

being the free space wave number, or propagation constant, $\omega$ being angular frequency, I being the unit dyad, and

$$
r=\left(x^{2}+y^{2}\right)^{\frac{1}{2}} .
$$

The scattered electric field $\mathbf{E}^{\mathbf{s}}$ at $\mathbf{z}=0$, due to the excitation of incident field $\mathbf{E}^{\mathbf{1}}$, can been derived from $\mathbf{A}$ [45] as:

$$
\boldsymbol{E} \cdot(x, y)=\frac{1}{j \omega \epsilon_{0}}\left[\begin{array}{ll}
\frac{\partial^{2}}{\partial x^{2}}+k_{0}^{2} & \frac{\partial}{\partial x} \frac{\partial}{\partial y} \\
\frac{\partial}{\partial x} \frac{\partial}{\partial y} & \frac{\partial^{2}}{\partial y^{2}}+k_{0}^{2}
\end{array}\right] \boldsymbol{A}(x, y)
$$


Fourier transforming equation (5) and substituting equation (1) into it, the relevant spectral domain equation is

$$
\tilde{\boldsymbol{E}} \boldsymbol{*}\left(k_{x}, k_{y}\right)=\frac{1}{j \omega \epsilon_{0}}\left[\begin{array}{cc}
k_{0}^{2}-k_{x}^{2} & -k_{x} k_{y} \\
-k_{x} k_{y} & k_{0}^{2}-k_{y}^{2}
\end{array}\right] \tilde{\boldsymbol{\sigma}}\left(k_{x}, k_{y}\right) \tilde{\boldsymbol{J}}\left(k_{x}, k_{y}\right),
$$

where " " means the Fourier transformed quantity. The Green's function also becomes

$$
\tilde{G}\left(k_{x}, k_{y}\right)=\frac{-j}{2\left(k_{0}^{2}-k_{x}^{2}-k_{y}^{2}\right)} I,
$$

$\mathrm{k}_{\mathrm{x}}, \mathrm{k}_{\mathrm{y}}$ are the transform variables corresponding to the $\mathrm{x}, \mathrm{y}$ coordinates, respectively.

Assume the transverse incident wave form

$$
\mathbb{E}^{1} e^{-j k \boldsymbol{r}}
$$

with

$$
\boldsymbol{r}=x \hat{x}+y \hat{y}
$$

the position vector in the transverse direction, the wave number vector becomes:

$$
\begin{aligned}
\boldsymbol{k} & =k_{0} \sin \theta \cos \phi \hat{x}+k_{0} \sin \theta \sin \phi \hat{y} \\
& =k_{x 0} \hat{x}+k_{y 0} \hat{y}
\end{aligned}
$$

where $\mu_{0}$ and $\varepsilon_{0}$ are the free space permeability and permittivity, respectively, and $\phi$ and $\theta$ are the polar and azimuthal angles of the incident field, respectively (see Figure 1.).

Since the frequency selective surface is doubly periodic, the Fourier transform of the induced current $\mathbf{J}$ has a discrete spectrum, i.e., $\mathbf{J}\left(\mathrm{k}_{\mathrm{x}}, \mathrm{k}_{\mathrm{y}}\right)$ is nonzero for discrete values 
of $k_{x}$ and $k_{y}$, namely, $k_{x m n}$ and $k_{y m n}$, which correspond to the Floquet modes [46]. The expressions for the Floquet modes $\mathrm{k}_{\mathrm{xmn}}$ and $\mathrm{k}_{\mathrm{ymn}}$ are

$$
\begin{aligned}
& k_{x m n}=\frac{2 \pi m}{a}+k_{0} \sin \theta \cos \phi, \\
& k_{y m n}=\frac{2 \pi n}{b}+k_{0} \sin \theta \sin \phi,
\end{aligned}
$$

where $-\infty<\mathrm{m}, \mathrm{n}<\infty$.

To simplify the notation, the subscripts $\mathrm{m}$ and $\mathrm{n}$ for $\mathrm{k}_{\mathrm{x}}$ and $\mathrm{k}_{\mathrm{y}}$ are usually eliminated. Also, equation (6) is rewritten in terms of the dyadic Green's function as follows, to consist with the following chapters' expressions.

$$
\tilde{\boldsymbol{E}^{\prime}}\left(k_{x}, k_{y}\right)=\left[\begin{array}{ll}
\tilde{G}_{x x}\left(k_{x}, k_{y}\right) & \tilde{G}_{x y}\left(k_{x}, k_{y}\right) \\
\tilde{G}_{y x}\left(k_{x}, k_{y}\right) & \tilde{G}_{y y}\left(k_{x}, k_{y}\right)
\end{array}\right] \tilde{\boldsymbol{J}}\left(k_{x}, k_{y}\right),
$$

or

$$
\left[\begin{array}{l}
\tilde{E}_{x}^{s}\left(k_{x}, k_{y}\right) \\
\tilde{E}_{y}^{s}\left(k_{x}, k_{y}\right)
\end{array}\right]=\left[\begin{array}{ll}
\tilde{G}_{x x}\left(k_{x}, k_{y}\right) & \tilde{G}_{x y}\left(k_{x}, k_{y}\right) \\
\tilde{G}_{y x}\left(k_{x}, k_{y}\right) & \tilde{G}_{y y}\left(k_{x}, k_{y}\right)
\end{array}\right]\left[\begin{array}{l}
\tilde{J}_{x}\left(k_{x}, k_{y}\right) \\
\tilde{J}_{y}\left(k_{x}, k_{y}\right)
\end{array}\right],
$$

where

$$
\left[\begin{array}{ll}
\tilde{G}_{x x}\left(k_{x}, k_{y}\right) & \tilde{G}_{x y}\left(k_{x}, k_{y}\right) \\
\tilde{G}_{y x}\left(k_{x}, k_{y}\right) & \tilde{G}_{y y}\left(k_{x}, k_{y}\right)
\end{array}\right]=-\frac{\eta_{0}}{2 k_{0}}\left[\begin{array}{cc}
\frac{k_{0}^{2}-k_{x}^{2}}{\left(k_{0}^{2}-k_{x}^{2}-k_{y}^{2}\right)^{\frac{1}{2}}} & \frac{-k_{x} k_{y}}{\left(k_{0}^{2}-k_{x}^{2}-k_{y}^{2}\right)^{\frac{1}{2}}} \\
\frac{-k_{x} k_{y}}{\left(k_{0}^{2}-k_{x}^{2}-k_{y}^{2}\right)^{\frac{1}{2}}} & \frac{k_{0}^{2}-k_{y}^{2}}{\left(k_{0}^{2}-k_{x}^{2}-k_{y}^{2}\right)^{\frac{1}{2}}}
\end{array}\right]
$$


$\eta_{0}$ is the characteristic impedance of free space.

On the patch, the boundary condition

$$
E^{1}+E^{2}=0
$$

must be satisfied. Thus, the following expression can be obtained from equation (14) and (16),

$$
-\left[\begin{array}{l}
\tilde{E}_{x}^{i}\left(k_{x}, k_{y}\right) \\
\tilde{E}_{y}^{i}\left(k_{x}, k_{y}\right)
\end{array}\right]=\left[\begin{array}{ll}
\tilde{G}_{x x}\left(k_{x}, k_{y}\right) & \tilde{G}_{x y}\left(k_{x}, k_{y}\right) \\
\tilde{G}_{y x}\left(k_{x}, k_{y}\right) & \tilde{G}_{y y}\left(k_{x}, k_{y}\right)
\end{array}\right]\left[\begin{array}{l}
\tilde{J}_{x}\left(k_{x}, k_{y}\right) \\
\tilde{J}_{y}\left(k_{x}, k_{y}\right)
\end{array}\right] .
$$

With equation (17), the scattering problem of free-standing frequency selective surface is ready to be solved by the method of moments.

\subsection{THE METHOD OF MOMENTS}

Before the wide application of high speed computers, one has to make some special effort to manipulate solutions analytically into a form to reduce the subsequent computations. With the aid of modern computers, one can conveniently use the methods which are analytically simple, but require large amounts of computation. Many problems of practical interests can now be solved by the use of such methods.

The method of moments is such an approach that its concept is to reduce a functional equation into a matrix one, and then solve the matrix equation with known 
techniques. A lot of numerical approximation techniques can be interpreted by the method of moments. For example, the classical eigenfunction approach corresponds to the particular choice of eigenfunctions for expansion and testing. The method of moments becomes the Galerkin's method when the test functions are chosen to be the same as the basis functions. The method of moments can be performed either in the time domain or in the spectral domain. The method of moments in the spectral domain is selected as the primary theoretical method in the dissertation. This choice is based upon its great versatility and advanced state of development. The method of moments has been applied to wider variety of problems than any other techniques.

To solve equation (17) with the method of moments, the first thing is to approximate the induced current density $\mathbf{J}$ by the basis functions. In selecting the basis functions, the following factors need to be considered. First, the basis functions must satisfy the appropriate edge conditions. Second, they must be analytically Fourier transformable. Third, the transforms of the basis functions must decay reasonably rapidly with large $\mathrm{k}_{\mathrm{x}}$ and $\mathrm{k}_{\mathrm{y}}$. Roof-top subdomain basis functions can satisfy the above selecting criteria [10], [15], and [8].

\subsection{BASIS FUNCTIONS AND TEST FUNCTIONS}

In using the method of moments, the appropriate choice of basis function is very important. Different basis functions may be used for different geometry patches. Usually, 
the entire domain basis functions are simpler to use than subdomain basis functions. But for some complex shape patches or more accurate solutions, subdomain basis functions may be used. The most commonly used subdomain basis functions for frequency selective surfaces are the roof-top, the surface patch, the triangular patches and the piecewise sinusoid. As the roof-top functions are with the simplest expressions and spend less computer CPU time [8] than other basis functions, it is used here.

Figure 2 shows how the roof-top functions can be used to represent the surface current density. These functions have a triangular distribution in one direction and a pulse distribution in the other [10]:

$$
B_{x}(p, q)=\Lambda\left(p+\frac{1}{2}\right) \Pi(q),
$$

and

$$
B_{y}(p, q)=\Pi(p) \Lambda\left(q+\frac{1}{2}\right),
$$

where

$$
\Lambda(m)=\left\{\begin{array}{cc}
1-\frac{|\xi-m \Delta \xi|}{\Delta \xi} & |\xi-m \Delta \xi| \leq \Delta \xi \\
0 & \text { elsewhere }
\end{array}\right.
$$

is the triangle function,

$$
\Pi(n)= \begin{cases}1 & |\xi-n \Delta \xi| \leq \frac{\Delta \xi}{2} \\ 0 & \text { elsewhere }\end{cases}
$$




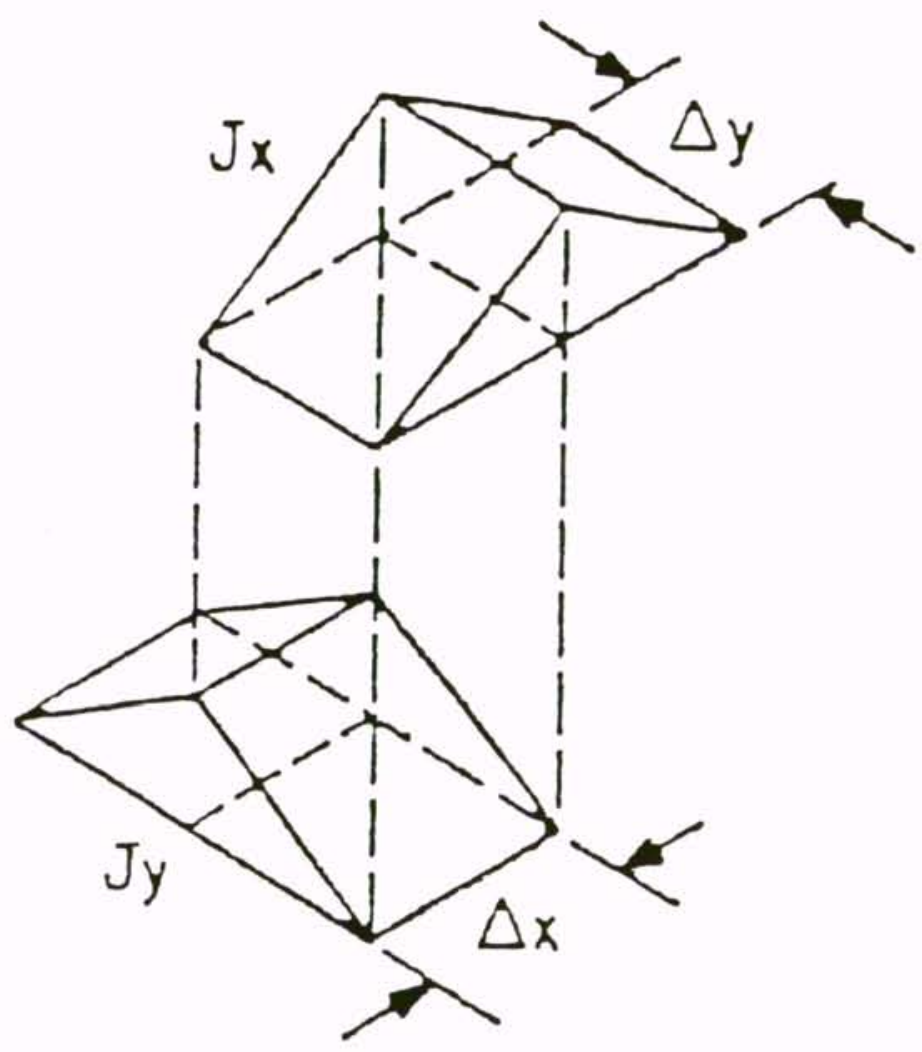

Figure 2. Geometry of roof -toping structure subdomain basis function. 
is the pulse function.

The functions overlap in such a way that the variation of the $\mathrm{x}$ component current density is piece-linear in the $\mathrm{x}$ direction and a step function in the $\mathrm{y}$ direction. Similarly, the variation of the $\mathrm{y}$ component current density is piece-linear in the $\mathrm{y}$ direction and a step function in the $\mathrm{x}$ direction. Every rectangular element $\Delta \mathrm{x} \Delta \mathrm{y}$ in the discrete unit cell is overlapped at least by one $\mathrm{x}$-direction roof-top and at least by one $\mathrm{y}$-direction roof-top. The roof-top approximation is consistent with the edge conditions on the patch.

Assume that the unit cell is divided by $\mathrm{M}$ in $\mathrm{x}$ direction and $\mathrm{N}$ in $\mathrm{y}$ direction, then

$$
\begin{aligned}
& a=M \Delta x, \\
& b=N \Delta y .
\end{aligned}
$$

The induced current density on one patch can be written as

$$
\begin{aligned}
& J_{x}=\sum_{p=-\frac{M}{2}}^{\frac{M}{2}-1} \sum_{q=-\frac{N}{2}}^{\frac{N}{2}-1} B_{x}(p, q) I_{x}(p, q), \\
& J_{y}=\sum_{p=-\frac{M}{2}}^{\frac{M}{2}-1} \sum_{q=-\frac{N}{2}}^{\frac{N}{2}-1} B_{y}(p, q) I_{y}(p, q) .
\end{aligned}
$$

Since the distribution of the induced current density on the patches is periodic, it can be expressed by a Fourier series, with the following Fourier series pair equations.

$$
x(t)=\sum_{k=-\infty}^{\infty} c_{k} e^{j \frac{2 \pi k}{T} t},
$$




$$
C_{k}=\frac{1}{T} \int_{T} X(t) e^{\frac{-j 2 \pi k}{T} t} d t,
$$

where $\mathrm{T}$ is the period.

Then the Fourier coefficients of the induced current density take the form

$$
\begin{aligned}
& \tilde{J}_{x}(m, n)=\frac{1}{a b} \sum_{p^{*}-\frac{M}{2}}^{\frac{M}{2}-1} \sum_{q=-\frac{N}{2}}^{\frac{N}{2}-1} \int_{p a t c h} B_{x}(p, q) e^{-j\left(k_{x=1} x+k_{y y} y\right)} d x d y I_{x}(p, q), \\
& \tilde{J}_{y}(m, n)=\frac{1}{a b} \sum_{p=-\frac{M}{2}}^{\frac{M}{2}-1} \sum_{q=-\frac{N}{2}}^{\frac{N}{2}-1} \int_{p a t c h} B_{y}(p, q) e^{-j\left(k_{x=1} x+k_{y y} y\right)} d x d y I_{y}(p, q) .
\end{aligned}
$$

But

$$
\begin{aligned}
& \int_{\text {patch }} B_{x}(p, q) e^{-j\left(k_{x a m} x+k_{y n} y\right)} d x d y=\int_{-\frac{a}{2}}^{\frac{a}{2}} \Lambda\left(p+\frac{1}{2}\right) e^{-j k_{x \rightarrow m} x} d x \int_{-\frac{b}{2}}^{\frac{b}{2}} \Pi(q) e^{-j k_{y x} y} d y \\
& =\int_{-\Delta x}^{\Delta x} \Lambda(0) e^{-j k_{x x+1} x} d x \int_{-\frac{\Delta y}{2}}^{\frac{\Delta y}{2}} \Pi(0) e^{-j k_{y z} y} d y e^{-j k_{x x}\left(p+\frac{1}{2}\right) \Delta x} e^{-j k_{y y} g \Delta y} \\
& =\Delta x \Delta y \operatorname{sinc}^{2}\left(\frac{k_{x m} \Delta x}{2}\right) \operatorname{sinc}\left(\frac{k_{y n} \Delta y}{2}\right) e^{-j k_{x m}\left(p+\frac{1}{2}\right) \Delta x} e^{-j k_{y n} q \Delta y} \\
& =\Delta x \Delta y \tilde{B}_{x}(m, n) P\left(p+\frac{1}{2}, q\right) \text {, }
\end{aligned}
$$

where

$$
\tilde{B}_{x}(m, n)=\operatorname{sinc}^{2}\left(\frac{k_{x m} \Delta x}{2}\right) \operatorname{sinc}\left(\frac{k_{y n} \Delta y}{2}\right),
$$




$$
\operatorname{sinc}(\xi)=\frac{\sin (\xi)}{\xi},
$$

and

$$
P(p, q)=e^{-j k_{\infty} p \Delta x} e^{-j k_{m} q \Delta y} .
$$

Similarly,

$$
\int_{p a t c h} B_{y}(p, q) e^{-j\left(k_{\Delta o x} x+k_{0} y\right)} d x d y=\Delta x \Delta y \tilde{B}_{y}(m, n) P\left(p, q+\frac{1}{2}\right),
$$

where

$$
\tilde{B}_{y}(m, n)=\operatorname{sinc}\left(\frac{k_{x m} \Delta x}{2}\right) \operatorname{sinc} c^{2}\left(\frac{k_{y n} \Delta y}{2}\right) .
$$

Then, equation (28) and (29) can be expressed as

$$
\begin{aligned}
{\left[\begin{array}{c}
\tilde{J}_{x}(m, n) \\
\tilde{J}_{y}(m, n)
\end{array}\right]=} & \frac{\Delta x \Delta y}{a b} \sum_{p=-\frac{M}{2}}^{\frac{M}{2}-1} \sum_{q=-\frac{M}{2}}^{\frac{N}{2}-1}\left[\begin{array}{cc}
\tilde{B}_{x}(m, n) & 0 \\
0 & \tilde{B}_{y}(m, n)
\end{array}\right] \\
& {\left[\begin{array}{cc}
P\left(p, q+\frac{1}{2}\right) & 0 \\
0 & P\left(p+\frac{1}{2}, q\right)
\end{array}\right]\left[\begin{array}{c}
I_{x}(p, q) \\
I_{y}(p, q)
\end{array}\right] }
\end{aligned}
$$




\subsection{MATRIX EQUATION OF THE SCATTERED FIELD}

Substituting equation (36) into (17), results in:

$$
\begin{aligned}
-\left[\begin{array}{c}
\tilde{E}_{x}^{\eta} \\
\tilde{E}_{y}^{i}
\end{array}\right]=\frac{\Delta x \Delta y}{a b} \sum_{p=-\frac{M}{2}}^{\frac{M}{2}-1} \sum_{q=-\frac{N}{2}}^{\frac{N}{2}-1}\left[\begin{array}{cc}
\tilde{G}_{x x}(m, n) & \tilde{G}_{x y}(m, n) \\
\tilde{G}_{y x}(m, n) & \tilde{G}_{y y}(m, n)
\end{array}\right]\left[\begin{array}{cc}
\tilde{B}_{x}(m, n) & 0 \\
0 & \tilde{B}_{y}(m, n)
\end{array}\right] \\
{\left[\begin{array}{cc}
P\left(p, q+\frac{1}{2}\right) & 0 \\
0 & P\left(p+\frac{1}{2}, q\right)
\end{array}\right]\left[\begin{array}{l}
I_{x}(p, q) \\
I_{y}(p, q)
\end{array}\right] . }
\end{aligned}
$$

Taking the inverse Fourier transform of equation (37) and changing the order of the summations yields

$$
\begin{aligned}
&-\left[\begin{array}{c}
E_{x}^{i} \\
E_{y}^{i}
\end{array}\right]=\frac{\Delta x \Delta y}{a b} \sum_{p=-\frac{M}{2}}^{\frac{M}{2}-1} \sum_{q=-\frac{N}{2}}^{\frac{N}{2}-1} \sum_{m=-\infty}^{\infty} \sum_{n=-\infty}^{\infty}\left[\begin{array}{ll}
\tilde{G}_{x x}(m, n) & \tilde{G}_{x y}(m, n) \\
\tilde{G}_{y x}(m, n) & \tilde{G}_{y y}(m, n)
\end{array}\right]\left[\begin{array}{cc}
\tilde{B}_{x}(m, n) & 0 \\
0 & \tilde{B}_{y}(m, n)
\end{array}\right] \\
& {\left[\begin{array}{cc}
P\left(p, q+\frac{1}{2}\right) & 0 \\
0 & P\left(p+\frac{1}{2}, q\right)
\end{array}\right]\left[\begin{array}{l}
I_{x}(p, q) \\
I_{y}(p, q)
\end{array}\right] e^{j\left(k_{x+x} x+k_{x y} y\right)} . }
\end{aligned}
$$

Multiplying the test functions to the above equation (38) and taking the complex inner products [8], the main expression for the analysis of frequency selective surface can be written as 


$$
\begin{gathered}
\sum_{p=-\frac{M}{2}}^{\frac{M}{2}-1} \sum_{q=-\frac{N}{2}}^{\frac{N}{2}-1} \sum_{m=-\infty}^{\infty} \sum_{n=-\infty}^{\infty}\left\{\left[\begin{array}{ll}
{\left[\tilde{Z}_{x x}(p, q)_{m n}\right.} & \tilde{Z}_{x y}(p, q)_{m n} \\
\tilde{Z}_{y x}(p, q)_{m n} & \tilde{Z}_{y y}(p, q)_{m n}
\end{array}\right] e^{-J\left[k_{x m}(p-r) \Delta x+k_{j m}(q-s) \Delta y\right]}\right\}\left[\begin{array}{l}
I_{x}(p, q) \\
I_{y}(p, q)
\end{array}\right] \\
=\left[\begin{array}{l}
E_{x 0} \tilde{T}_{x}^{*}(0,0) P_{00}\left(r, s+\frac{1}{2}\right) \\
E_{y 0} \tilde{T}_{y}^{*}(0,0) P_{00}\left(r+\frac{1}{2}, s\right)
\end{array}\right], \quad-\frac{M}{2}<r<\frac{M}{2}-1, \quad-\frac{N}{2}<s<\frac{N}{2}-1
\end{gathered}
$$

where

$$
\begin{gathered}
\tilde{Z}_{x x}(p, q)_{m n}=-\frac{\Delta x \Delta y}{a b} \tilde{G}_{x x}(m, n) \tilde{B}_{x}(m, n) \tilde{T}_{x}^{*}(m, n), \\
\tilde{Z}_{x y}(p, q)_{m n}=-\frac{\Delta x \Delta y}{a b} \tilde{G}_{x y}(m, n) \tilde{B}_{y} \tilde{T}_{x}^{*}(m, n) e^{j k_{x m} \frac{\Delta x}{2}} e^{-j k_{j m} \frac{\Delta y}{2},} \\
\tilde{Z}_{x x}(p, q)_{m n}=-\frac{\Delta x \Delta y}{a b} \tilde{G}_{x y}(m, n) \tilde{B}_{x} \tilde{T}_{y}^{*}(m, n) e^{-j k_{x m} \frac{\Delta x}{2}} e^{j k_{y x} \frac{\Delta y}{2}}, \\
\tilde{Z}_{y y}(p, q)_{m n}=-\frac{\Delta x \Delta y}{a b} \tilde{G}_{y y}(m, n) \tilde{B}_{y}(m, n) \tilde{T}_{y}^{*}(m, n),
\end{gathered}
$$

"*" means complex conjugate quantities. Equation (39) can be solved by matrix inversion. Since the Galerkin's method is used here, the test functions are chosen to be the same as the basis functions.

Although equation (39) is derived for the free-standing case, it is also suitable for the situation of frequency selective surface with substrates. The only parts that need to be modified is the Green's function, and the equivalent incident electric fields (see Chapter 4). 


\subsection{REFLECTION COEFFICIENTS}

Having derived the Green's function for free-standing frequency selective surfaces, one can calculate the induced current density on the patches by the method of moments. Knowing the current distribution, one may also simulate the scattered fields. While a plane wave incident on the structure of Fig. 1, gives rise to an infinite number of scattered wave modes, only a finite number of these propagate, the others being evanescent. If the frequencies are restricted such that $\min (\mathrm{a}, \mathrm{b})<\lambda / 2$, only the $(0,0)$ mode can propagate. The amplitude of this mode is highly dependent on the polarization of the incident field. Thus the incident field is decomposed into TE and TM polarization components. Similarly, the scattered field is also decomposed into TE and TM polarization components.

For the incident wave number

$$
\boldsymbol{k}_{t}^{i}=k_{0}(\sin \theta \cos \phi \hat{x}+\sin \theta \sin \phi \hat{y}),
$$

the vector

$$
A=\cos \phi \hat{x}+\sin \phi \hat{y},
$$

is the unit vector along $\mathbf{k}_{1}{ }^{i}$ in the transverse plane, and

$$
B=\sin \phi \hat{x}-\cos \phi \hat{y},
$$

is the unit vector perpendicular to $\mathbf{A}$.

With unit vectors $\mathbf{A}$ and $\mathbf{B}$, the incident wave with angle $\theta$ to the $\mathrm{z}$ coordinate can be decomposed into 


$$
H_{t}^{(I E)}=H_{t}(\cos \phi \hat{x}+\sin \phi \hat{y}),
$$

$$
H_{t}^{(T M)}=H_{t}(\sin \phi \hat{x}-\cos \phi \hat{y}) .
$$

Equations (47) and (48) is also suitable for the scattered fields.

Because of the presence of the apertures, an incident wave of one polarization will excite scattered waves with both polarizations. There are four possible reflection coefficient expressions, two for each polarization.

$$
\begin{aligned}
& R_{T M}^{T M}=-\left.\frac{H_{D 00}^{s(T M)}}{H_{t}^{i(T M)}}\right|_{z=0^{+}} \\
& R_{T E}^{T E}=-\left.\frac{H_{t 00}^{s(T E)}}{H_{t}^{i(T E)}}\right|_{z=0^{*}} \\
& R_{T M}^{T E}=-\left.\frac{H_{D 00}^{s(T E)}}{H_{t}^{i(T M)}}\right|_{z=0^{+}} \\
& R_{T E}^{T M}=-\left.\frac{H_{t 00}^{s(T M)}}{H_{t}^{i(T E)}}\right|_{z=0^{+}},
\end{aligned}
$$

where equations (49) and (50) are referred to as the co-polarization reflection coefficients, and equations (51) and (52) are referred to as the cross-polarization reflection coefficients. 
For the free-standing situation, the structure is symmetric for both sides of the frequency selective surface, so the boundary condition on the patch becomes

$$
-\hat{z} \times J_{m n}=2 H_{t m n}^{s}\left(z=0^{+}\right),
$$

where $\mathbf{J}_{\mathrm{mn}}$ is the $(\mathrm{m}, \mathrm{n})$ th component of the induced current density on the patch, $\boldsymbol{H}_{\mathrm{tm}}^{\boldsymbol{B}}\left(\boldsymbol{z}=0^{+}\right)$is the transverse part of the $(\mathrm{m}, \mathrm{n})$ th component of the scattered magnetic field just above the conductor.

With the known incident magnetic field and the scattered magnetic field expressed by equation (53), as well as equations (47)and (48), the reflection coefficients in (49)-(52) are easily calculated. 


\section{CHAPTER 3 \\ PROPERTIES OF FERRITE MATERIALS}

Ferrites are a group of materials with a particular type of crystal structure [35], which have the characteristics of low loss, strong magnetic effects at microwave frequencies [47] and a permeability of anisotropic behavior when the ferrite is subject to a DC magnetic field [48]. The properties of ferrites will be discussed here by examining the propagation of microwave electromagnetic waves in an unbounded ferrite material. A brief description of the microscopic view at a ferrite will also be given in this chapter. Much of the discussion of this chapter follows that of [48].

\subsection{PERMEABILITY TENSORS}

The magnetic properties of a material is determined by its magnetic dipole moments, including both orbital and spin motions. Although the orbital motion may contribute to the total magnetic moment in a free atom, its contribution in the solid is often small and sometimes negligible. This means the electron spin is the principal contributor to the magnetic properties of the solid. In this section, the permeability tensors of ferrite materials will be derived. 
Usually, opposite sign electron spins appear by pair in solids. The overall magnetic moment is negligible. In a magnetic material, a large amount of the electron spins are oriented in a random fashion so that the net magnetic moment is still small. When an external magnetic field is applied to the magnetic material, the magnetic dipoles will tend to align in the same direction as the applied magnetic field to produce a large magnetic moment.

In addition to spin moment, a spinning electron also has a spin angular momentum, when a magnetic bias field $H_{0}=2 H_{0}$ is present. If the magnetic dipole moment of an electron due to its spin is expressed as $\mathbf{m}$ and the spin angular momentum as $\mathbf{J}$, they are related by the following equation

$$
\boldsymbol{m}=-\boldsymbol{\gamma} \boldsymbol{J},
$$

where $\gamma$ is called the gyromagnetic ratio, $\gamma=1.759 \times 10^{11} \mathrm{C} / \mathrm{Kg}$. A torque will be exerted on the magnetic dipole due to its rotation

$$
T=\frac{d J}{d t} .
$$

Considering that the torque is equal to the magnetic moment cross multiplying magnetic flux density and taking equation (54) into account,

$$
T=m \times B_{0}=\mu_{0} \boldsymbol{m} \times H_{0}=\mu_{0} \gamma J \times H_{0} .
$$

Torque is also equal to the time rate of change of angular momentum. Then the equation of motion for the magnetic dipole moment is given as follows: 


$$
\frac{d m}{d t}=-\gamma \boldsymbol{T}=-\mu_{0} \gamma \boldsymbol{m} \times \boldsymbol{H}_{0}
$$

The direction of above vectors are indicated in Figure 3. The magnetic dipole $\mathbf{m}$ will precesses around the $\mathbf{H}_{0}$ vector continuously, if there is no any damping forces. The actual precession angle will be determined by the initial position of the magnetic dipole. In reality, there are damping forces existing which will force the magnetic dipole moment to spiral in from its initial angle until $\mathbf{m}$ is aligned with $\mathbf{H}_{0}(\theta=0)$.

Now, considering $\mathrm{N}$ unbalanced electron spins (magnetic dipoles) in the unit volume, the total magnetization becomes:

$$
\boldsymbol{M}=N \boldsymbol{m},
$$

and equation (57) becomes

$$
\frac{d \boldsymbol{M}}{d t}=-\mu_{0} \gamma \boldsymbol{M} \times \boldsymbol{H}
$$

where $\mathbf{H}$ is the total internal applied field (including bias field and any other applied fields). With the increase of the bias field $\mathrm{H}_{0}$, the magnetization $\mathbf{M}$ will also increase until all the magnetic dipole moments are aligned with $\mathrm{H}_{0}$, and $\mathbf{M}$ reaches its upper limit. Then the material is said to be magnetically saturated, and $M_{s}$ is called as the saturation magnetization. Figure 4 shows the curve $M_{s}$ versus $H_{0}$. Since ferrite materials can be very lossy below saturation at microwave frequencies, they are usually operated in the saturated state. The unit for magnetic field $\mathrm{H}_{0}$ is $\mathrm{A} / \mathrm{m}$ with MKS units, Oersted with CGS units. $4 \pi \times 10^{-3}$ Oersted $=1 \mathrm{~A} / \mathrm{m}$. The unit for magnetization is Weber $/ \mathrm{m}^{2}$ with $\mathrm{MKS}$ units, Gauss for $4 \pi \mathrm{M}_{\mathrm{s}} .1$ Gauss $=10^{-4} \mathrm{Weber} / \mathrm{m}^{2}$. In practice, CGS units are used more often 


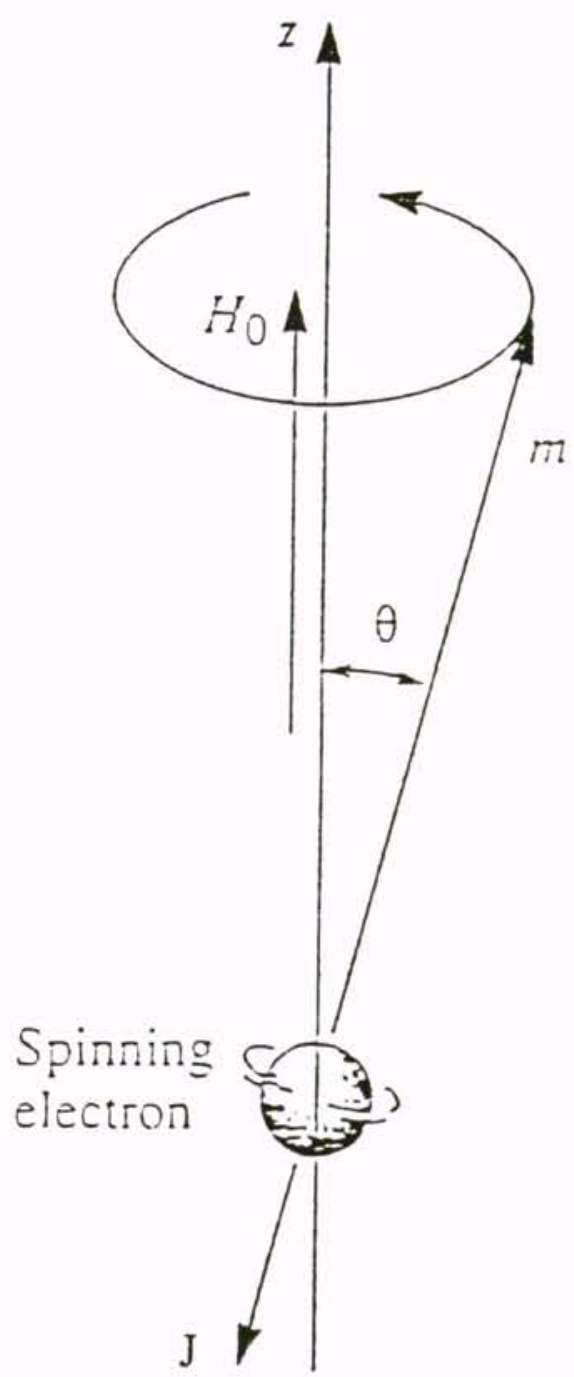

Figure 3. Spinning electron with spin magnetic dipole moment and angular. 


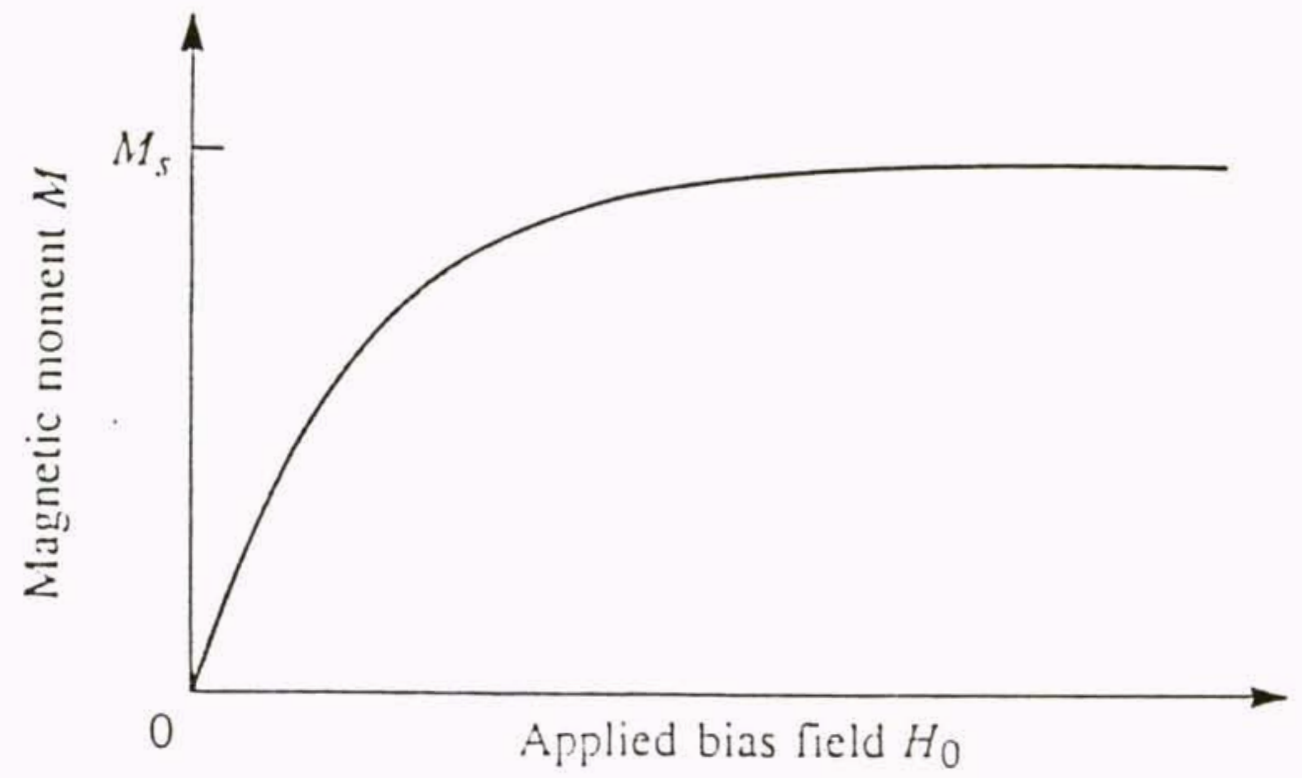

Figure 4. Magnetic moment of a ferrite material versus bias field $\mathrm{H}_{0}$. 
than MKS one.

Assume $\mathbf{H}$ to be the applied AC field, $\mathrm{H}_{0}$ to be the bias field, the total magnetic field then becomes

$$
H_{t}=H_{0} 2+H,
$$

where $|\boldsymbol{H}|<H_{0}$ is assumed. So the total magnetization in the ferrite material must have the form

$$
M_{t}=M_{s} z+M
$$

where $M_{3}$ is the DC saturation magnetization and $\mathbf{M}$ is the additional AC magnetization caused by $\mathbf{H}$. Equations (60) and (61) are substituted into equation (59) and all products of $\mathrm{AC}$ terms are considered to be negligible compared with the products involving one steady term and one $\mathrm{AC}$ term. The results are

$$
\begin{gathered}
\frac{d M_{x}}{d t}=-\omega_{0} M_{y}+\omega_{m} H_{y \prime} \\
\frac{d M_{y}}{d t}=\omega_{0} M_{x}-\omega_{m r} H_{x} \\
\frac{d M_{z}}{d t}=0,
\end{gathered}
$$

where $\omega_{0}=\mu_{0} \gamma H_{0}$ is the Larmor, or precession, frequency, and $\omega_{m}=\mu_{0} \gamma M_{s}$. Solving equation (62) and (63) for $M_{x}$ and $M_{y}$ gives

$$
\frac{d^{2} M_{x}}{d t^{2}}+\omega_{0}^{2} M_{x}=\omega_{m} \frac{d H_{y}}{d t}+\omega_{0} \omega m H_{x}
$$




$$
\frac{d^{2} M_{y}}{d t^{2}}+\omega_{0}^{2} M_{y}=-\omega_{m} \frac{d H_{x}}{d t}+\omega_{0} \omega_{m} H_{y} .
$$

Assume the AC $\mathbf{H}$ field has a time convention $e^{j \omega t}$, the phasor forms simplify equations (65) and (66) as follows

$$
\begin{aligned}
& \left(\omega_{0}^{2}-\omega^{2}\right) M_{x}=\omega_{0} \omega_{m} H_{x}+j \omega \omega_{m} H_{y^{\prime}} \\
& \left(\omega_{0}^{2}-\omega^{2}\right) M_{y}=-j \omega \omega_{m} H_{x}+\omega_{0} \omega_{m} H_{y} .
\end{aligned}
$$

As $\mathbf{H}$ and $\mathbf{M}$ are linearly related, equations (67) and (68) can be written with a susceptibility tensor form as:

$$
\boldsymbol{M}=[\chi] \boldsymbol{H}=\left[\begin{array}{ccc}
\chi_{x x} & \chi_{x y} & 0 \\
\chi_{y x} & \chi_{y y} & 0 \\
0 & 0 & 0
\end{array}\right] \boldsymbol{H}
$$

where the elements of $[\chi]$ are given by

$$
\begin{aligned}
& \chi_{x x}=\chi_{y y}=\frac{\omega_{0} \omega_{m}}{\omega_{0}^{2}-\omega^{2}}, \\
& \chi_{x y}=-\chi_{y x}=\frac{j \omega \omega_{m}}{\omega_{0}^{2}-\omega^{2}} .
\end{aligned}
$$

With equations of (69), (70) and (71), the relation between $\mathbf{B}$ and $\mathbf{H}$ is

$$
B=\mu_{0}(\boldsymbol{M}+H)=[\mu] H,
$$


where the permeability tensor $[\boldsymbol{\mu}]$ has the form

$$
[\mu]=\mu_{0}([I]+[\chi])=\mu_{0}\left[\begin{array}{ccc}
\mu & j \kappa & 0 \\
-j \kappa & \mu & 0 \\
0 & 0 & 1
\end{array}\right] \quad(z \text { bias }) .
$$

The elements of the permeability tensor are then

$$
\begin{gathered}
\mu=1+\chi_{x x}=1+\chi_{y y}=1+\frac{\omega_{0} \omega_{m}}{\omega_{0}^{2}-\omega^{2}}, \\
\kappa=-j \chi_{x y}=j \chi_{y x}=\frac{\omega \omega_{m}}{\omega_{0}^{2}-\omega^{2}} .
\end{gathered}
$$

A material having a permeability tensor with the form of equation (73) is called gyrotropic.

Similarly, if the ferrite is biased in different directions, the permeability tensors can be written as

$$
\begin{aligned}
& {[\mu]=\mu_{0}\left[\begin{array}{ccc}
1 & 0 & 0 \\
0 & \mu & j \kappa \\
0 & -j \kappa & \mu
\end{array}\right] \quad(\widehat{x} \text { bias }),} \\
& {[\mu]=\mu_{0}\left[\begin{array}{ccc}
\mu & 0 & j \kappa \\
0 & 1 & 0 \\
-j \kappa & 0 & \mu
\end{array}\right] \quad(y \text { bias }) .}
\end{aligned}
$$

From equations (74) and (75), the ferrite material has its own resonant frequency. The resonant frequency is equal to the precession frequency:

$$
\omega_{0}=\mu_{0} \gamma H_{0} .
$$


This resonant frequency will be useful to explain the physical phenomena of the frequency selective surfaces with ferrite substrates.

\subsection{THE INTERACTION OF AN AC SIGNAL WITH A BIASED FERRITE}

Suppose a right-hand circularly polarized field with phasor form

$$
\boldsymbol{H}^{\boldsymbol{r}}=H^{r}(\hat{x}-j \hat{y}) \text {. }
$$

Applying the above RHCP field to equation (67) and (68), results in the magnetization components

$$
M_{x}^{r}=\frac{\omega_{m}}{\omega_{0}-\omega} H^{r}
$$

and

$$
M_{y}^{r}=\frac{-j \omega_{m}}{\omega_{0}-\omega} H^{r}
$$

The vector magnetization expression is

$$
\boldsymbol{M}^{\boldsymbol{r}}=M_{x}^{r} \hat{x}+M_{y}^{r} \hat{y}=\frac{\omega_{m}}{\omega_{0}-\omega} H^{r}(\hat{x}-j \hat{y})
$$

It is seen that the magnetization is also RHCP and rotates around the z-axis with the same angular velocity $\omega$ as the driving field $\mathbf{H}^{\mathrm{r}}$ (see Figure 5). Thus, the magnetic flux density can be written as 


$$
B^{x}=\mu_{0}\left(M^{x}+H^{x}\right)=\mu^{r} H^{x},
$$

where $\mu^{r}$ is the effective permeability for a RHCP wave with the expression

$$
\mu^{r}=\mu_{0}\left(1+\frac{\omega_{m}}{\omega_{0}-\omega}\right) .
$$

The magnetic dipole precesses in the same direction as its free precession (see Figure 3).

For a left-hand circularly polarized field, the magnetic field has the phasor form

$$
H^{1}=H^{1}(\hat{x}+j \hat{y}) \text {. }
$$

Applying equation (85) to equations (67) and (68), gives the magnetization component expressions

$$
M_{x}^{1}=\frac{\omega_{m}}{\omega_{0}+\omega} H^{1},
$$

and

$$
M_{y}^{1}=\frac{j \omega_{m}}{\omega_{0}+\omega} H^{1}
$$

The expression of the vector magnetization is

$$
\boldsymbol{M}^{1}=M_{x}^{1} \hat{x}+M_{y}^{1} \hat{y}=\frac{\omega_{m}}{\omega_{0}+\omega} H^{1}(\hat{x}+j \hat{y}) .
$$

It is seen that the magnetization is LHCP and rotates around the z-axis with the same 


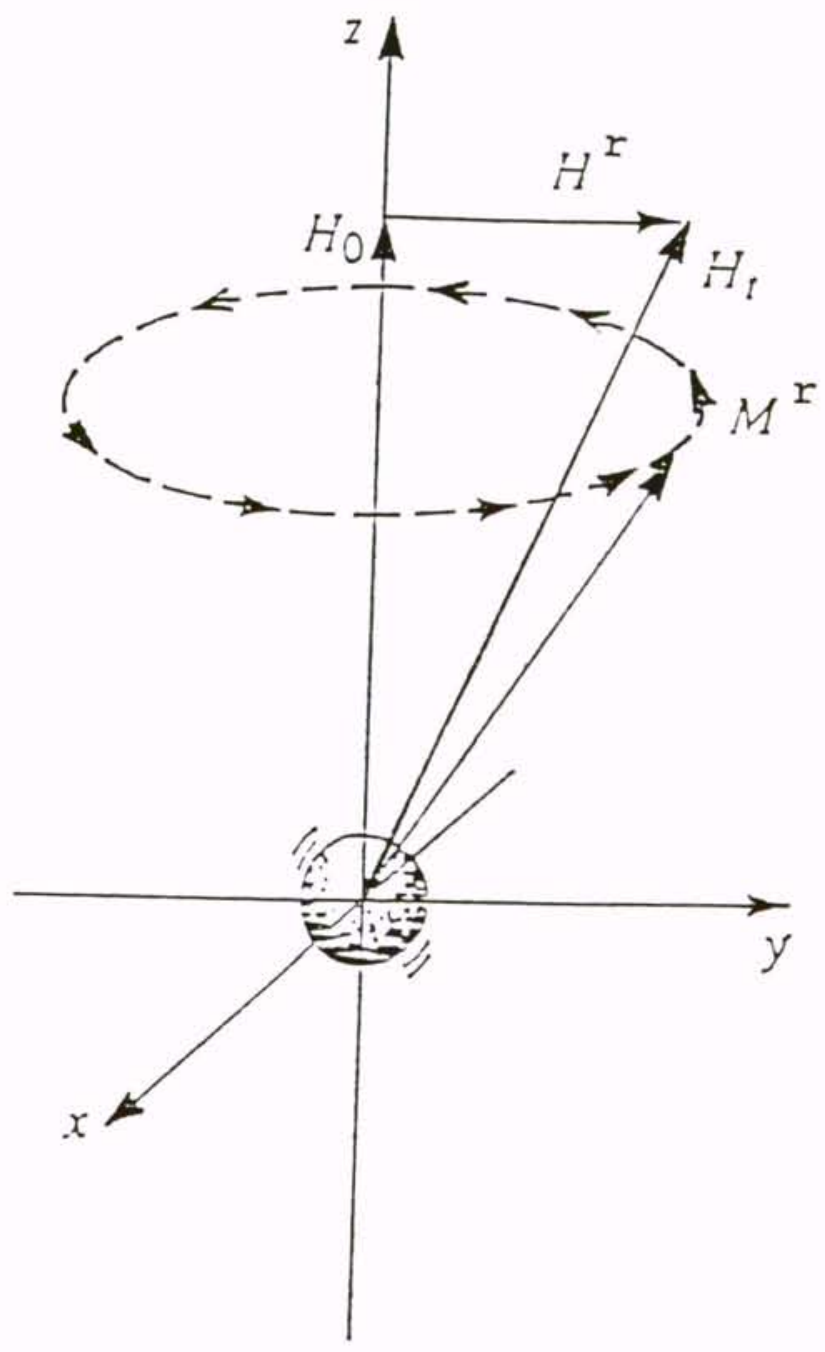

Figure 5. Forced precession of a magnetic dipole with right hand circular polarization (RHCP). 
angular velocity $\omega$ as the driving field $\mathbf{H}^{1}$ (see Figure 6). The magnetic flux density can be written as

$$
B^{1}=\mu_{0}\left(M^{1}+H^{1}\right)=\mu^{1} H^{1},
$$

where $\mu^{1}$ is the effective permeability for a LHCP wave with the expression

$$
\mu^{1}=\mu_{0}\left(1+\frac{\omega_{m}}{\omega_{0}+\omega}\right)
$$

The magnetic dipole here precesses in the opposite direction to its free precession (see Figure 3).

It is seen that the affect of a biased ferrite to a circularly polarized wave depends on its polarization (RHCP or LHCP). The free precession set up by the bias field coincides with the direction of forced precession for a RHCP wave but is opposite to that of a LHCP wave. The degree of the deviation between the RHCP and LHCP waves is determined by the bias field and the frequency of the AC signal. This effect leads to nonreciprocal propagation characteristics.

\subsection{PLANE WAVE PROPAGATION IN FERRITE MEDIUM}

In Section 3.1, the macroscopic expressions (permeability tensors) that describe the microscopic phenomena occurring inside a biased ferrite material were derived. With expressions (73), (76) and (77), Maxwell's equations can be solved for waves propagating 


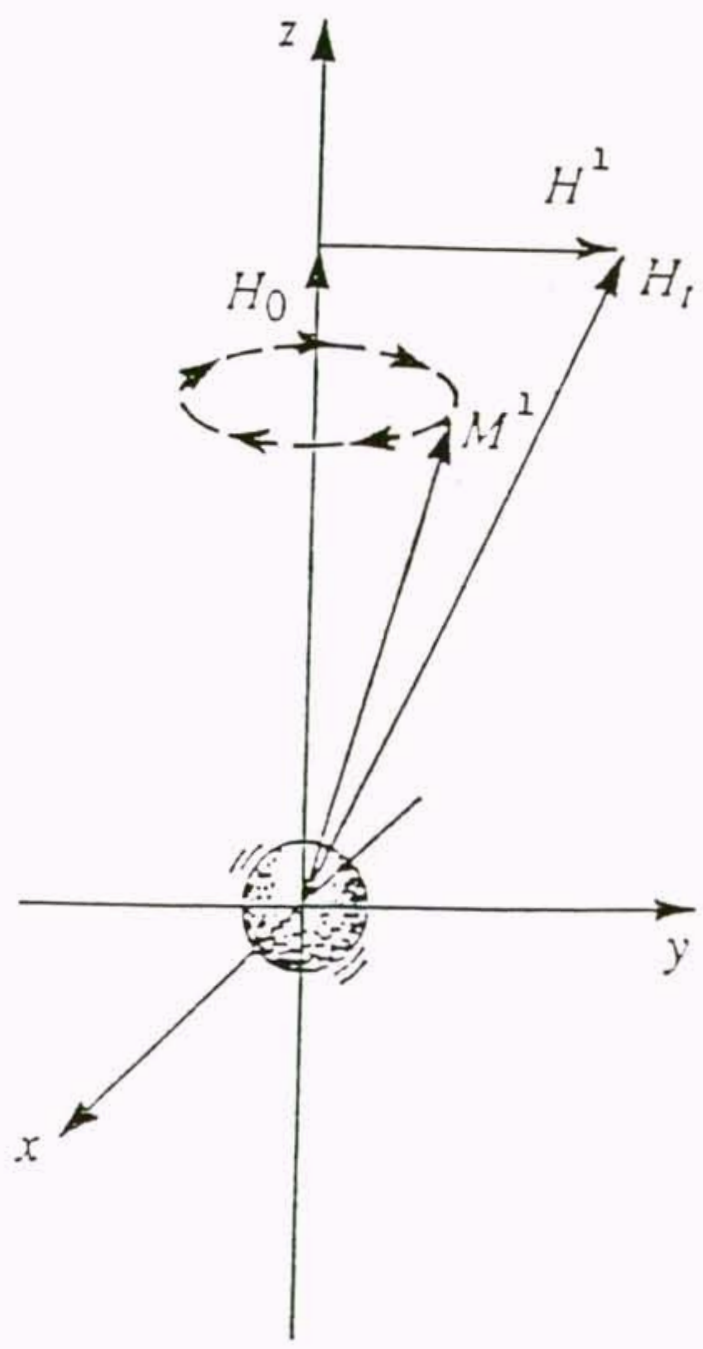

Figure 6. Forced precession of a magnetic dipole with left hand circular polarization (LHCP). 
in ferrite medium. To illustrate the important effects of Faraday rotation and birefringence, the problems of plane wave propagating either in the direction of bias or propagating transverse to the bias field will be investigated.

\subsubsection{FARADAY ROTATION}

Consider the case of an infinite ferrite region with a DC bias field given in $\mathrm{z}$ direction (in the direction of the wave propagation). The permeability tensor is given by equation (73). Maxwell's equations can be written as

$$
\begin{gathered}
\nabla \times E=-j \omega[\mu] H \\
\nabla \times H=j \omega \epsilon E \\
\nabla \cdot D=0
\end{gathered}
$$

$$
\nabla \cdot B=0
$$

Then assume that the plane wave propagates in the $\mathrm{z}$ direction. The electric and magnetic fields are only functions of $\mathrm{z}$ with the following forms:

$$
\begin{aligned}
& \boldsymbol{E}=\boldsymbol{E}_{0} e^{-j k z}, \\
& \boldsymbol{H}=\boldsymbol{H}_{0} e^{-j k z} .
\end{aligned}
$$

Expanding equations (91)-(94) and solving them, results in

$$
j \omega^{2} \epsilon \kappa E_{x}+\left(k^{2}-\omega^{2} \mu \epsilon\right) E_{y}=0,
$$




$$
\left(k-\omega^{2} \mu \epsilon\right) E_{x}-j \omega^{2} \epsilon \kappa E_{y}=0 .
$$

For the nontrivial solutions of equations (97) and (98), the determinant of this set of equations must be zero:

$$
\omega^{4} \epsilon^{2} \kappa^{2}-\left(k^{2}-\omega^{2} \mu \epsilon\right)^{2}=0,
$$

or

$$
k_{ \pm}=\omega \sqrt{\epsilon(\mu \pm K)} .
$$

There are two possible propagation constants, $\mathrm{k}_{+}$and $\mathrm{k}_{+}$.

Applying $k_{+}$from equation (100) to equations (97) and (98) results in

$$
E_{y}=-j E_{x}
$$

Then the electric field of equation (95) must have the following form

$$
\boldsymbol{E}_{+}=E_{0}(\hat{x}-j \hat{y}) e^{-j k, z}
$$

This is a right-hand circular polarized (RHCP) plane wave.

Similarly, the fields related to k. are left-hand circularly polarized (LHCP) wave:

$$
E_{-}=E_{0}(\hat{x}+j \hat{y}) e^{-j k_{-} z} .
$$

It is then seen that RHCP and LHCP plane waves are the two modes existing in the ferrite medium with the $\mathrm{z}$ direction bias. They propagate through the ferrite medium with different propagation constants expressed by equation (100). The physical explanation for this effect is that the magnetic bias field creates a preferred direction for the magnetic 
dipole precession, that is to say, it is coincident with the RHCP wave but opposite to that of the LHCP wave.

In general, elliptically polarized waves can be decomposed into RHCP and LHCP waves of unequal amplitude. To simplify the analysis, considering a linearly polarized electric field, at $z=0$, is decomposed as a sum of a RHCP and a LHCP wave:

$$
\left.\boldsymbol{E}\right|_{z=0}=\hat{x} E_{0}=\frac{E_{0}}{2}(\hat{x}-j \hat{y})+\frac{E_{0}}{2}(\hat{x}+j \hat{y}) .
$$

According to the above analysis, the RHCP component will propagate in the $\mathrm{z}$ direction with propagation constant $k_{+}$, and the LHCP component will propagate in the same direction but with propagation constant $\mathbf{k}$. So the total field has the expression

$$
\begin{aligned}
& \boldsymbol{E}=\frac{E_{0}}{2}(\hat{x}-j \hat{y}) e^{-j k_{+} z}+\frac{E_{0}}{2}(\hat{x}+j \hat{y}) e^{-j k_{-} z} \\
& =\frac{E_{0}}{2} \hat{x}\left(e^{-j k_{+}+e^{-j k \cdot z}}\right)-j \frac{E_{0}}{2} y\left(e^{-j k_{*}}-e^{-j k_{-} z}\right) \\
& =E_{0}\left[\hat{x} \cos \left(\frac{k_{+}-k_{-}}{2}\right) z-\hat{y} \sin \left(\frac{k_{+}-k_{-}}{2}\right) z\right] e^{-j\left(k_{+}+k_{-}\right) z / 2} .
\end{aligned}
$$

Equation (105) is still a linearly polarized wave, but its polarization rotates as the wave propagates along the $z$-direction. This effect is called Faraday rotation.

\subsubsection{BIREFRINGENCE}

If a bias field is applied in the $\mathrm{x}$ direction (perpendicular to the direction of the wave propagation), the permeability tensor expression (76) should be used in Maxwell's equations. Solving equations (91)-(94), results in 


$$
\begin{gathered}
k^{2} E_{y}=\omega^{2} \mu_{0} \epsilon E_{y^{\prime}} \\
\mu\left(k^{2}-\omega^{2} \mu \epsilon\right) E_{x}=-\omega^{2} \epsilon \kappa^{2} E_{x} .
\end{gathered}
$$

The solution for equation (106) is

$$
k_{0}=\omega \sqrt{\mu_{0} \epsilon},
$$

with $\mathrm{E}_{\mathrm{x}}=0$. Then the electric field is

$$
E_{0}=\hat{y} E_{0} e^{-j k_{0} z}
$$

Since it is the same as the wave in free-space, and unaffected by the magnetization of the ferrite, it is called the ordinary $(O)$ wave.

The solution for equation (107) is

$$
k_{\theta}=\omega \sqrt{\mu_{e} \epsilon},
$$

with $\mathrm{E}_{\mathrm{y}}=0$. where $\mu_{\mathrm{e}}$ is an effective permeability given by

$$
\mu_{e}=\frac{\mu^{2}-\kappa^{2}}{\mu} \text {. }
$$

This wave is called the extraordinary (E) wave. It is equivalent to a wave propagating in the medium with permeability equal to the effective permeability. It is affected by the bias of the ferrite.

It is seen that for a plane wave propagating transverse to the $\mathrm{x}$ direction bias field of the ferrite medium, the electric field consists of a y component which is an ordinary 
wave and a $\mathrm{x}$ component which is an extraordinary wave. Such an effect is called birefringence.

In general, the wave in a ferrite material is composed of the right-hand circularly polarized (RHCP) and the left-hand circularly polarized (LHCP) modes when its propagation is parallel to the bias field, the ordinary $(\mathrm{O})$ and the extraordinary $(\mathrm{E})$ modes when the propagation is perpendicular to the bias field. Two of these modes (RHCP and E) are very strongly influenced by the magnetic properties of the medium. The propagation constant of the $\mathrm{E}$ (RHCP) deviates very much from the propagation constant, $\mathrm{k}_{0}$, of the $\mathrm{O}$. Under this situation, E and RHCP modes almost have the nature of magnetostatic wave (with small propagation constant). 


\section{CHAPTER 4}

\section{ANALYSIS OF FREQUENCY SELECTIVE SURFACES WITH FERRITE SUBSTRATES}

The general analysis procedure of free-standing frequency selective surface is given in Chapter 2. Mathematically, the main difference of analysis between the free-standing case and the non free-standing (dielectric or ferrite substrate) case is in their Green's functions.

\subsection{DERIVATION OF GREEN'S FUNCTION FOR FERRITE SUBSTRATE}

To derive the dyadic Green's function for frequency selective surface with ferrite substrate, the permeability tensor derived in the previous chapter is applied to Maxwell's equations as below

$$
\begin{gathered}
\nabla \times \bar{E}=-j \omega \boldsymbol{\mu} \bar{H}, \\
\nabla \times \bar{H}=j \omega \epsilon \bar{E} .
\end{gathered}
$$

Since there are different permeability tensor expressions for different bias directions, the 
Green's function must be derived separately for different bias directions. Without loss of generality, the cases with $y$ direction bias (in plane of the FSS) and $z$ direction bias (normal to the FSS) are considered. Figure 7(a) shows a y direction biased frequency selective surface.

First consider the $\mathrm{y}$ direction bias case. Substituting the $\mathrm{y}$ direction bias permeability tensor expression (77) in equation (112), in the spectral domain, yields:

$$
\begin{gathered}
{\left[\begin{array}{c}
j k_{y} \tilde{E}_{z}-\frac{\partial \tilde{E}_{y}}{\partial z} \\
\frac{\partial \tilde{E}_{x}}{\partial z}-j k_{x} \tilde{E}_{z} \\
j k_{x} \tilde{E}_{y}-j k_{y} \tilde{E}_{x}
\end{array}\right]=\left[\begin{array}{c}
-j \omega \mu_{0} \mu \tilde{H}_{x}+\omega \mu_{0} k \tilde{H}_{z} \\
-j \omega \mu_{0} \tilde{H}_{y} \\
-\omega \mu_{0} \kappa \tilde{H} x-j \omega \mu_{0} \mu \tilde{H}_{z}
\end{array}\right],} \\
{\left[\begin{array}{c}
j k_{y} \tilde{H}_{z}-\frac{\partial \tilde{H}_{y}}{\partial z} \\
\frac{\partial \tilde{H}_{x}}{\partial z}-j k_{x} \tilde{H}_{z} \\
j k_{x} \tilde{H}_{y}-j k_{y} \tilde{H}_{x}
\end{array}\right]=\left[\begin{array}{l}
j \omega \epsilon \tilde{E}_{x} \\
j \omega \epsilon \tilde{E}_{y} \\
j \omega \epsilon \tilde{E}_{z}
\end{array}\right] .}
\end{gathered}
$$

Solving $\tilde{E}_{y}$, equations (114) and (115) result in

$$
\frac{\partial^{4} \tilde{E}_{y}}{\partial z^{4}}-(A+B) \frac{\partial^{2} \tilde{E}_{y}}{\partial z^{2}}+(A B-C) \tilde{E}_{y}=0
$$

where

$$
\begin{gathered}
A=k_{x}^{2}+k_{y}^{2}-k_{e}^{2} \frac{\mu^{2}-\kappa^{2}}{\mu}, \\
B=k_{x}^{2}+\frac{1}{\mu} k_{y}^{2}-k_{e}^{2},
\end{gathered}
$$




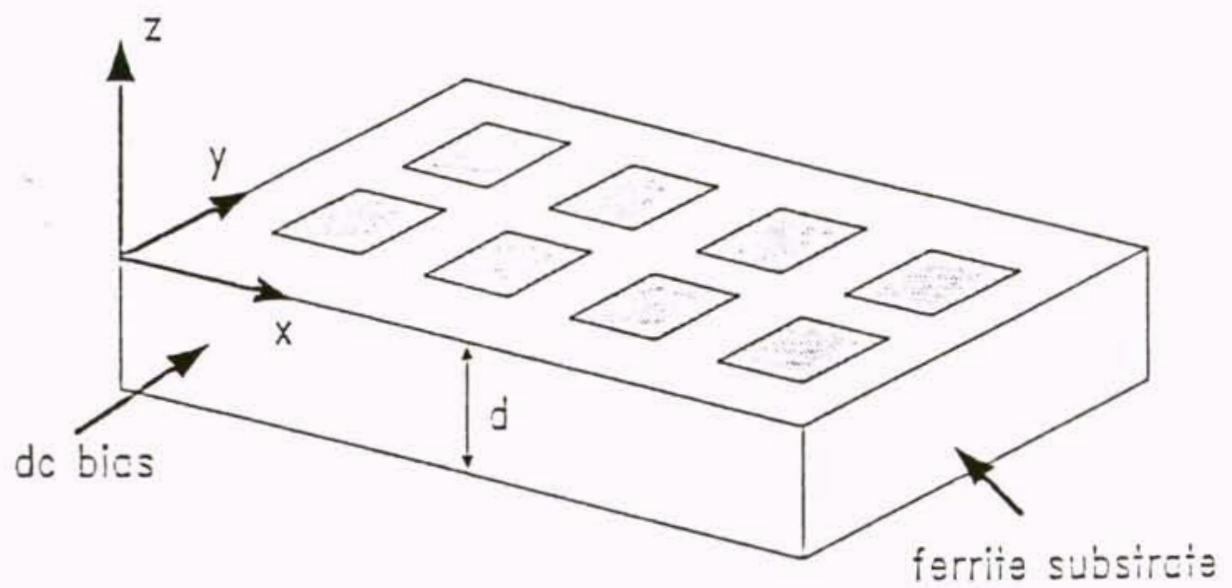

(a)

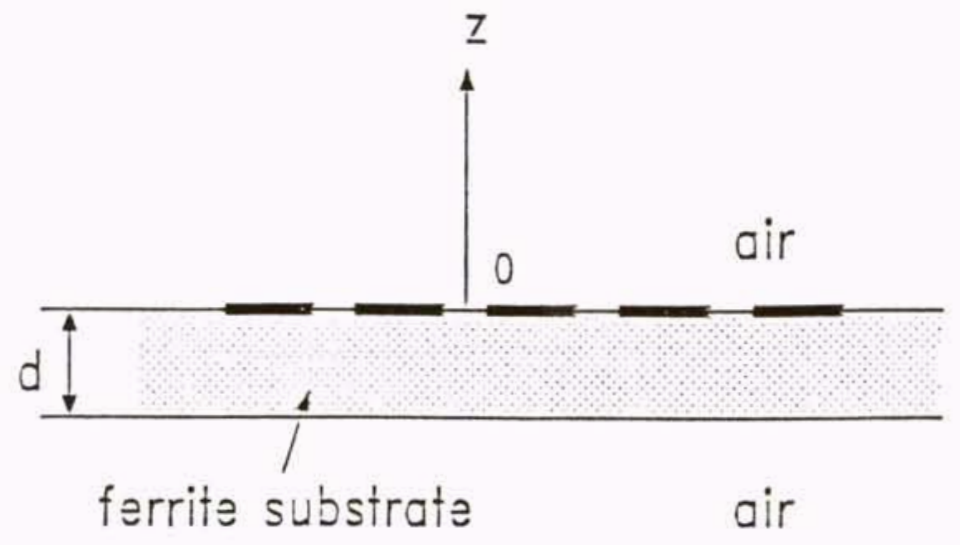

(b)

Figure 7. (a) Geometry of a frequency selective surface on ferrite substrate. (b) Sideview of a frequency selective surface on ferrite substrate. 


$$
C=\left(\frac{\kappa}{\mu} k_{\theta}^{2} k_{y}\right)^{2},
$$

and

$$
k_{\theta}^{2}=\omega^{2} \mu_{0} \epsilon .
$$

The general solution for the differential equation (116) is

$$
\tilde{E}_{y}=A_{1} e^{\lambda, z}+A_{2} e^{-\lambda, z}+A_{3} e^{\lambda-z}+A_{4} e^{-\lambda-z},
$$

where

$$
\lambda_{ \pm}^{2}=\frac{1}{2}\left[(A+B) \pm \sqrt{(A+B)^{2}-4(A B-C)}\right],
$$

are the four roots of equation (116). These four roots yield the well-known ordinary (O) and extraordinary (E) type solutions. $\tilde{E}_{x}, \tilde{H}_{x}$ and $\tilde{H}_{y}$ can be expressed in the similar way as equation (121)

$$
\begin{aligned}
& \tilde{E}_{x}=B_{1} e^{\lambda, z}+B_{2} e^{-\lambda, z}+B_{3} e^{\lambda_{-} z}+B_{4} e^{-\lambda_{-} z}, \\
& \tilde{H}_{x}=C_{1} e^{\lambda, z}+C_{2} e^{-\lambda, z}+C_{3} e^{\lambda_{-} z}+C_{4} e^{-\lambda_{-} z}, \\
& \tilde{H}_{y}=D_{1} e^{\lambda, z}+D_{2} e^{-\lambda_{2} z}+D_{3} e^{\lambda_{-} z}+D_{4} e^{-\lambda_{-} z} .
\end{aligned}
$$

With equations (114) and (115), the coefficients in equations (123), (124) and (125) can be expressed by the ones in equation (121), that is 


$$
\left[\begin{array}{cccc}
B_{1} & B_{2} & B_{3} & B_{4} \\
A_{1} & A_{2} & A_{3} & A_{4} \\
C_{1} & C_{2} & C_{3} & C_{4} \\
D_{1} & D_{2} & D_{3} & D_{4}
\end{array}\right]=\left[\begin{array}{cccc}
\alpha_{1} & \alpha_{2} & \alpha_{3} & \alpha_{4} \\
1 & 1 & 1 & 1 \\
\beta_{1} & \beta_{2} & \beta_{3} & \beta_{4} \\
\gamma_{1} & \gamma_{2} & \gamma_{3} & \gamma_{4}
\end{array}\right]\left[\begin{array}{c}
A_{1} \\
A_{2} \\
A_{3} \\
A_{4}
\end{array}\right]
$$

The detailed expressions of the elements in equation (126) are as follows

$$
\begin{aligned}
& \alpha_{1,2}=\frac{\mu k_{x} k_{y}^{3}-\mu^{2}\left(k_{e}^{2}-k_{x}^{2}\right) k_{x} k_{y}}{\mu\left(\mu^{2}-\kappa^{2}\right)\left(k_{e}^{2}-k_{x}^{2}\right) k_{\theta}^{2}-\mu^{2} k_{e}^{2} k_{y}^{2}+\mu k_{y}^{4}} \\
& \frac{ \pm \mu \kappa k_{e}^{2} k_{y} \lambda_{+}-\mu^{2} k_{x} k_{y} \lambda_{+}^{2}}{-\mu^{2}\left(k_{e}^{2}-k_{x}^{2}\right) k_{e}^{2}+\mu\left(\mu^{2}-\kappa^{2}\right) k_{\theta}^{2} \lambda_{+}^{2}-\mu k_{y}^{2} \lambda_{+}^{2}},
\end{aligned}
$$

where $\alpha_{1}$ takes "+" sign, $\alpha_{2}$ takes "-" sign. $\alpha_{3,4}$ have the same form as equation (127) with the exception of $\lambda_{+}$being replaced by $\lambda_{+}$.

$$
\begin{aligned}
\beta_{1,2} & =\frac{-j \omega \epsilon\left[\kappa k_{y}\left(k_{e}^{2}-k_{x}^{2}\right) \alpha_{1,2}-\kappa k_{x}\left(k_{e}^{2}-k_{x}^{2}\right)\right]}{\Delta} \\
& \mp \frac{j \omega \epsilon\left[\mu k_{x} k_{y} \lambda_{+} \alpha_{1,2} \pm \mu\left(k_{e}^{2}-k_{x}^{2}\right) \lambda_{+}\right]}{\Delta},
\end{aligned}
$$

where

$$
\Delta=k_{\theta}^{2}\left[\left(\mu^{2}-\kappa^{2}\right)\left(k_{\theta}^{2}-k_{x}^{2}\right)-\mu k_{y}^{2}\right],
$$

$\beta_{1}$ takes "+" sign, $\beta_{2}$ takes "-" sign. $\beta_{3,4}$ have the same form as equation (128) with the exceptions of $\lambda_{+}$being replaced by $\lambda_{*}$ and $\alpha_{1,2}$ being replaced by $\alpha_{3,4}$.

$$
\begin{aligned}
& \gamma_{1,2}= \\
& \frac{-j \omega \epsilon\left\{-\kappa k_{x} k_{y}^{2} \alpha_{1,2}+\kappa k_{x}^{2} k_{y} \mp\left[\left(\mu^{2}-\kappa^{2}\right) k_{\theta}^{2}-\mu k_{y}^{2}\right] \lambda_{+} \alpha_{1,2} \mp \mu k_{x} k_{y} \lambda_{+}\right\}}{\Delta},
\end{aligned}
$$


where $\gamma_{1}$ takes "-" sign, $\gamma_{2}$ takes "+" sign. $\gamma_{3,4}$ have the same form as equation (130) with the exceptions of $\lambda_{+}$being replaced by $\lambda_{-}$and $\alpha_{1,2}$ being replaced by $\alpha_{3,4}$.

Then, equations (121), (122), (123) and (124) can be written as

$$
\left[\begin{array}{c}
\tilde{E}_{x} \\
\tilde{E}_{y} \\
\tilde{H}_{x} \\
\tilde{H}_{y}
\end{array}\right]=\left[\begin{array}{cccc}
\alpha_{1} & \alpha_{2} & \alpha_{3} & \alpha_{4} \\
1 & 1 & 1 & 1 \\
\beta_{1} & \beta_{2} & \beta_{3} & \beta_{4} \\
\gamma_{1} & \gamma_{2} & \gamma_{3} & \gamma_{4}
\end{array}\right]\left[\begin{array}{cccc}
e^{\lambda, z} & 0 & 0 & 0 \\
0 & e^{-\lambda, z} & 0 & 0 \\
0 & 0 & e^{\lambda-z} & 0 \\
0 & 0 & 0 & e^{-\lambda-z}
\end{array}\right]\left[\begin{array}{c}
A_{1} \\
A_{2} \\
A_{3} \\
A_{4}
\end{array}\right],
$$

or symbolically

$$
\tilde{V}(z)=\tilde{\boldsymbol{M}} \boldsymbol{X}(z) \boldsymbol{A},
$$

where

$$
\begin{gathered}
\tilde{\boldsymbol{V}}(z)=\left[\begin{array}{c}
\tilde{E}_{x} \\
\tilde{E}_{y} \\
\tilde{H}_{x} \\
\tilde{H}_{y}
\end{array}\right], \\
\tilde{\boldsymbol{M}}=\left[\begin{array}{cccc}
\alpha_{1} & \alpha_{2} & \alpha_{3} & \alpha_{4} \\
1 & 1 & 1 & 1 \\
\beta_{1} & \beta_{2} & \beta_{3} & \beta_{4} \\
\gamma_{1} & \gamma_{2} & \gamma_{3} & \gamma_{4}
\end{array}\right], \\
\boldsymbol{X}(\boldsymbol{z})=\left[\begin{array}{cccc}
e^{\lambda . z} & 0 & 0 & 0 \\
0 & e^{-\lambda, z} & 0 & 0 \\
0 & 0 & e^{\lambda . z} & 0 \\
0 & 0 & 0 & e^{-\lambda . z}
\end{array}\right],
\end{gathered}
$$


and

$$
\boldsymbol{A}=\left[\begin{array}{c}
A_{1} \\
A_{2} \\
A_{3} \\
A_{4}
\end{array}\right] .
$$

Since equation (132) is applicable to the whole ferrite substrate, the boundaries of ferrite at $z=0$ and $z=-d$ must satisfy the equation (132) too. Thus

$$
\begin{gathered}
\tilde{\boldsymbol{V}}(0)=\tilde{\boldsymbol{M}} \boldsymbol{\Lambda}(0) \boldsymbol{A}, \\
\tilde{\boldsymbol{V}}(-d)=\tilde{\boldsymbol{M}} \boldsymbol{\Lambda}(-d) \boldsymbol{A} .
\end{gathered}
$$

Combining equations (137) and (138), the fields components relation between $z=0$ and $z=-d$ has the form

$$
\begin{aligned}
\tilde{\boldsymbol{V}}(0) & =\tilde{\boldsymbol{M}} \boldsymbol{X}(d) \tilde{\boldsymbol{M}}^{-1} \tilde{\boldsymbol{V}}(-d) \\
& =\tilde{\mathbf{T}}(d) \tilde{V}(-d),
\end{aligned}
$$

where

$$
\tilde{\boldsymbol{T}}(d)=\tilde{\boldsymbol{M}} \boldsymbol{\Lambda}(d) \tilde{\boldsymbol{M}}^{-1}
$$

is the transmission matrix of the ferrite substrate. Note that the transmission matrix is only a function of $\mathrm{d}$, and it is independent of the $\mathrm{z}$ coordinate.

So far, the description of the fields in the ferrite substrate is given by the transmission matrix. The next step is to cascade the ferrite substrate transmission matrix 
with the free space expressions above and below the substrate (see Figure 7 (b)).

It is known that a plane wave in linear and isotropic media can be decomposed into TE and TM components [46]. Since the boundary conditions for the transverse field components will be used late, either $\left(\mathrm{TE}_{\mathrm{x}}, \mathrm{TM}_{\mathrm{x}}\right)$ modes or $\left(\mathrm{TE}_{\mathrm{y}}, \mathrm{TM}_{\mathrm{y}}\right)$ modes can be used. Here, $\left(\mathrm{TE}_{\mathrm{x}}, \mathrm{TM}_{\mathrm{x}}\right)$ modes are chosen. In free space, the vector potential $\mathbf{A}$ defines fields

$$
\boldsymbol{H}=\nabla \times \boldsymbol{A}
$$

and

$$
\boldsymbol{E}=\frac{1}{j \omega \epsilon_{0}}\left(\nabla(\nabla \cdot \boldsymbol{A})+k_{0}^{2} \boldsymbol{A}\right)
$$

where

$$
k_{0}=\omega \sqrt{\mu_{0} \epsilon_{0}} .
$$

For $\mathrm{TM}_{\mathrm{x}}$ mode

$$
A=\hat{X} \psi ，
$$

and $\psi$ satisfy Laplace's equation

$$
\nabla^{2} \Psi+k_{0}^{2} \Psi=0
$$

Applying equation (144) into equation (141) and (142), the following expressions are obtained

$$
E_{x}=\frac{1}{j \omega \epsilon_{0}}\left(\frac{\partial^{2} \Psi}{\partial x^{2}}+k_{0}^{2} \Psi\right),
$$




$$
\begin{gathered}
E_{y}=\frac{1}{j \omega \epsilon_{0}} \frac{\partial^{2} \psi}{\partial x \partial y}, \\
E_{z}=\frac{1}{j \omega \epsilon_{0}} \frac{\partial^{2} \psi}{\partial x \partial z}, \\
H_{x}=0, \\
H_{y}=\frac{\partial \psi}{\partial z}, \\
H_{z}=-\frac{\partial \psi}{\partial y} .
\end{gathered}
$$

In the spectral domain, equations (147)-(151) have the forms of

$$
\begin{gathered}
\tilde{E}_{x}=\frac{1}{j \omega \epsilon_{0}}\left(k_{0}^{2}-k_{x}^{2}\right) \Psi, \\
\tilde{E}_{y}=-\frac{k_{x} k_{y}}{j \omega \epsilon_{0}} \Psi_{,} \\
\tilde{E}_{z}=-\frac{j k_{x}}{j \omega \epsilon_{0}} \tilde{\Psi}_{z^{\prime}}
\end{gathered}
$$

where the subscript $z$ of $\tilde{\Psi}$ means the differential to $z$,

$$
\begin{aligned}
& \tilde{H}_{x}=0, \\
& \tilde{H}_{y}=\tilde{\Psi}_{z}
\end{aligned}
$$




$$
\tilde{H}_{z}=j k_{y} \Psi
$$

In the spectral domain, equation (145) becomes

$$
\Psi_{z z}+\left(k_{0}^{2}-k_{x}^{2}-k_{y}^{2}\right) \Psi=0
$$

The general solution of equation (158) is

$$
\Psi=G_{1} e^{\lambda_{0} z}+G_{2} e^{-\lambda_{0} z}
$$

where $G_{1}$ and $G_{2}$ are the unknown constants determined by the boundary conditions,

$$
\lambda_{0}=\sqrt{k_{x}^{2}+k_{y}^{2}-k_{0}^{2}}
$$

Thus,

$$
\begin{gathered}
\tilde{E}_{x}=\frac{k_{0}^{2}-k_{x}^{2}}{j \omega \epsilon_{0}}\left(G_{1} e^{\lambda_{0} z}+G_{2} e^{-\lambda_{0} z}\right), \\
\tilde{E}_{y}=-\frac{k_{x} k_{y}}{j \omega \epsilon_{0}}\left(G_{1} e^{\lambda_{0} z}+G_{2} e^{-\lambda_{0} z}\right), \\
\tilde{H}_{x}=0, \\
\tilde{H}_{y}=\lambda_{0}\left(G_{1} e^{\lambda_{0} z}+G_{2} e^{-\lambda_{0} z}\right) .
\end{gathered}
$$


The $\mathrm{TE}_{\mathrm{x}}$ mode has the similar expressions as follows

$$
\begin{gathered}
\boldsymbol{E}=-\nabla \times \boldsymbol{F}, \\
\boldsymbol{H}=\frac{1}{j \omega \mu_{0}}\left[\nabla(\nabla \cdot \boldsymbol{F})+k_{0}^{2} \boldsymbol{F}\right], \\
\boldsymbol{F}=\hat{x} \boldsymbol{\phi},
\end{gathered}
$$

and

$$
\nabla^{2} \phi+k_{0}^{2} \phi=0
$$

The fields expressions in the spectral domain are

$$
\begin{gathered}
\tilde{E}_{x}=0, \\
\tilde{E}_{y}=-\lambda_{0}\left(G_{3} e^{\lambda_{0} z}-G_{4} e^{-\lambda_{0} z}\right), \\
\tilde{H}_{x}=\frac{k_{0}^{2}-k_{x}^{2}}{j \omega \mu_{0}}\left(G_{3} e^{\lambda_{0} z}+G_{4} e^{-\lambda_{0} z}\right), \\
\tilde{H}_{y}=-\frac{k_{x} k_{y}}{j \omega \mu_{0}}\left(G_{3} e^{\lambda_{0} z}+G_{4} e^{-\lambda_{0} z}\right) .
\end{gathered}
$$

With the equations (161)-(164) and (169)-(172), the total field components in free space can be written as 


$$
\left[\begin{array}{c}
\tilde{E}_{x} \\
\tilde{E}_{y} \\
\tilde{H}_{x} \\
\tilde{H}_{y}
\end{array}\right]=\left[\begin{array}{cccc}
\frac{k_{0}^{2}-k_{x}^{2}}{j \omega \epsilon_{0}} & \frac{k_{0}^{2}-k_{x}^{2}}{j \omega \epsilon_{0}} & 0 & 0 \\
-\frac{k_{x} k_{y}}{j \omega \epsilon_{0}} & -\frac{k_{x} k_{y}}{j \omega \epsilon_{0}} & -\lambda_{0} & \lambda_{0} \\
0 & 0 & \frac{k_{0}^{2}-k_{x}^{2}}{j \omega \mu_{0}} & \frac{k_{0}^{2}-k_{x}^{2}}{j \omega \mu_{0}} \\
\lambda_{0} & -\lambda_{0} & -\frac{k_{x} k_{y}}{j \omega \mu_{0}} & -\frac{k_{x} k_{y}}{j \omega \mu_{0}}
\end{array}\right]
$$

or symbolically

$$
\tilde{V}(z)=\tilde{M}_{0} X_{0}(z) G
$$

where $G_{1}, G_{2}, G_{3}$ and $G_{4}$ are the unknown constants determined by the boundary conditions. Equation (173) is suitable for both above and below the substrate.

In the case of above the substrate, $G_{1}=G_{3}=0$, this means that the scattered fields are outgoing waves. Equation (173) becomes 


$$
\left[\begin{array}{c}
\tilde{E}_{x} \\
\tilde{E}_{y} \\
\tilde{H}_{x} \\
\tilde{H} y_{y}
\end{array}\right]=\left[\begin{array}{cccc}
\frac{k_{0}^{2}-k_{x}^{2}}{j \omega \epsilon_{0}} & \frac{k_{0}^{2}-k_{x}^{2}}{j \omega \epsilon_{0}} & 0 & 0 \\
-\frac{k_{x} k_{y}}{j \omega \epsilon_{0}} & -\frac{k_{x} k_{y}}{j \omega \epsilon_{0}} & -\lambda_{0} & \lambda_{0} \\
0 & 0 & \frac{k_{0}^{2}-k_{x}^{2}}{j \omega \mu_{0}} & \frac{k_{0}^{2}-k_{x}^{2}}{j \omega \mu_{0}} \\
\lambda_{0} & -\lambda_{0} & -\frac{k_{x} k_{y}}{j \omega \mu_{0}} & -\frac{k_{x} k_{y}}{j \omega \mu_{0}}
\end{array}\right]
$$

For the same reason, $G_{2}=G_{4}=0$ for the fields below the ferrite substrate. Equation (173) becomes

$$
\left[\begin{array}{c}
\tilde{E}_{x} \\
\tilde{E}_{y} \\
\tilde{H}_{x} \\
\tilde{H}_{y}
\end{array}\right]=\left[\begin{array}{cccc}
\frac{k_{0}^{2}-k_{x}^{2}}{j \omega \epsilon_{0}} & \frac{k_{0}^{2}-k_{x}^{2}}{j \omega \epsilon_{0}} & 0 & 0 \\
-\frac{k_{x} k_{y}}{j \omega \epsilon_{0}} & -\frac{k_{x} k_{y}}{j \omega \epsilon_{0}} & -\lambda_{0} & \lambda_{0} \\
0 & 0 & \frac{k_{0}^{2}-k_{x}^{2}}{j \omega \mu_{0}} & \frac{k_{0}^{2}-k_{x}^{2}}{j \omega \mu_{0}} \\
\lambda_{0} & -\lambda_{0} & -\frac{k_{x} k_{y}}{j \omega \mu_{0}} & -\frac{k_{x} k_{y}}{j \omega \mu_{0}}
\end{array}\right]
$$


here the primes for $G_{1}$ and $G_{3}$ represent the unknown constants below the substrate.

Considering the boundary conditions below

$$
\begin{gathered}
\tilde{\boldsymbol{v}}\left(0_{+}\right)-\tilde{\boldsymbol{V}}\left(0_{-}\right)=\left[\begin{array}{c}
0 \\
0 \\
\tilde{J}_{y} \\
-\tilde{J}_{x}
\end{array}\right], \\
\tilde{v}\left(-d_{+}\right)=\tilde{V}\left(-d_{-}\right) .
\end{gathered}
$$

$\tilde{\boldsymbol{v}}\left(0_{-}\right)$can be written as

$$
\tilde{\boldsymbol{V}}\left(0_{-}\right)=\tilde{\boldsymbol{T}}(d) \tilde{M}_{0} \boldsymbol{X}_{0}(-d)\left[\begin{array}{c}
G_{1} \\
0 \\
G_{3}^{\prime} \\
0
\end{array}\right] \text {. }
$$

$\tilde{\boldsymbol{V}}\left(0_{+}\right)$is

$$
\tilde{V}\left(0_{+}\right)=\tilde{M}_{0}\left[\begin{array}{c}
0 \\
G_{2} \\
0 \\
G_{4}
\end{array}\right] \text {. }
$$

Combining equations (179) and (180) with the boundary condition (177), the dyadic Green's function for the frequency selective surface with ferrite substrate is ready to be given as 


$$
\left[\begin{array}{c}
\tilde{E}_{x}^{s}\left(0_{+}\right) \\
\tilde{E}_{y}^{s}\left(0_{+}\right)
\end{array}\right]=\left[\begin{array}{ll}
\tilde{G}_{x x}\left(k_{x}, k_{y}\right) & \tilde{G}_{x y}\left(k_{x}, k_{y}\right) \\
\tilde{G}_{y x}\left(k_{x}, k_{y}\right) & \tilde{G}_{y y}\left(k_{x}, k_{y}\right)
\end{array}\right]\left[\begin{array}{c}
\tilde{J}_{x} \\
\tilde{J}_{y}
\end{array}\right] .
$$

The detailed expressions for the Green's function is given in Appendix A.

Similarly, when the ferrite substrate is biased in $z$ direction, permeability tensor expression (73) is used. Then Maxwell's equations in spectral domain become

$$
\left[\begin{array}{c}
j k_{y} \tilde{E}_{z}-\frac{\partial \tilde{E}_{y}}{\partial z} \\
\frac{\partial \tilde{E}_{x}}{\partial z}-j k_{x} \tilde{E}_{z} \\
j k_{x} \tilde{E}_{y}-j k_{y} \tilde{E}_{x}
\end{array}\right]=\left[\begin{array}{c}
-j \omega \mu_{0} \mu \tilde{H}_{x}+\omega \mu_{0} k \tilde{H}_{y} \\
-\omega \mu_{0} k \tilde{H}_{x}-j \omega \mu_{0} \mu \tilde{H}_{y} \\
-j \omega \mu_{0} \tilde{H}_{z}
\end{array}\right],
$$

$$
\left[\begin{array}{c}
j k_{y} \tilde{H}_{z}-\frac{\partial \tilde{H}_{y}}{\partial z} \\
\frac{\partial \tilde{H}_{x}}{\partial z}-j k_{x} \tilde{H}_{z} \\
j k_{x} \tilde{H}_{y}-j k_{y} \tilde{H}_{x}
\end{array}\right]=\left[\begin{array}{c}
j \omega \epsilon \tilde{E}_{x} \\
j \omega \epsilon \tilde{E}_{y} \\
j \omega \epsilon \tilde{E}_{z}
\end{array}\right]
$$

Solving $\tilde{H}_{y}$ with equation (182) and (183), the fourth order differential equation is obtained

$$
\begin{gathered}
\frac{\partial^{4} \tilde{H}_{y}}{\partial z^{4}}+\left[2 \mu k_{e}^{2}-(\mu+1)\left(k_{x}^{2}-k_{y}^{2}\right)\right] \frac{\partial^{2} \tilde{H}_{y}}{\partial z^{2}}+\mu\left(k_{e}^{2}-k_{x}^{2}-k_{y}^{2}\right) \\
\left(\frac{\mu^{2}-k^{2}}{\mu} k_{e}^{2}-k_{x}^{2}-k_{y}^{2}\right) \tilde{H}_{y}=0 .
\end{gathered}
$$

The general solution for equation (184) is

$$
\tilde{H}_{y}=A_{1} e^{\lambda \cdot z}+A_{2} e^{-\lambda, z}+A_{3} e^{\lambda_{-} z}+A_{4} e^{-\lambda_{-} z},
$$


where

$$
\begin{aligned}
& \lambda_{ \pm}^{2}=\frac{1}{2}\left\{(\mu+1)\left(k_{x}^{2}+k_{y}^{2}\right)-2 \mu k_{\theta}^{2} \pm\right. \\
& \left.\sqrt{\left[2 \mu k_{\theta}^{2}-(\mu+1)\left(k_{x}^{2}+k_{y}^{2}\right)\right]^{2}-4 \mu\left(k_{\theta}^{2}-k_{x}^{2}-k_{y}^{2}\right)\left(\frac{\mu^{2}-k^{2}}{\mu} k_{\theta}^{2}-k_{x}^{2}-k_{y}^{2}\right)}\right\} \text {. }
\end{aligned}
$$

Again, $\tilde{E}_{x}, \tilde{E}_{y}, \tilde{H}_{x}$ are written in the similar forms as equation (185):

$$
\begin{aligned}
& \tilde{E}_{x}=C_{1} e^{\lambda, z}+C_{2} e^{-\lambda, z}+C_{3} e^{\lambda-z}+C_{4} e^{-\lambda . z}, \\
& \tilde{E}_{y}=D_{1} e^{\lambda, z}+D_{2} e^{-\lambda, z}+D_{3} e^{\lambda-z}+D_{4} e^{-\lambda_{-} z}, \\
& \tilde{H}_{x}=B_{1} e^{\lambda, z}+B_{2} e^{-\lambda, z}+B_{3} e^{\lambda_{-} z}+B_{4} e^{-\lambda_{-} z} .
\end{aligned}
$$

The coefficients in equations (187), (188) and (189) can be linearly expressed by the ones in equation (185) through equations (182) and (183),

$$
\left[\begin{array}{cccc}
C_{1} & C_{2} & C_{3} & C_{4} \\
D_{1} & D_{2} & D_{3} & D_{4} \\
B_{1} & B_{2} & B_{3} & B_{4} \\
A_{1} & A_{2} & A_{3} & A_{4}
\end{array}\right]=\left[\begin{array}{cccc}
\beta_{1} & \beta_{2} & \beta_{3} & \beta_{4} \\
\gamma_{1} & \gamma_{2} & \gamma_{3} & \gamma_{4} \\
\alpha_{1} & \alpha_{2} & \alpha_{3} & \alpha_{4} \\
1 & 1 & 1 & 1
\end{array}\right]\left[\begin{array}{c}
A_{1} \\
A_{2} \\
A_{3} \\
A_{4}
\end{array}\right]
$$

The detailed expression for equation (190) is given in Appendix B. Then the transverse fields components can be written as

$$
\left[\begin{array}{c}
\tilde{E}_{x} \\
\tilde{E}_{y} \\
\tilde{H}_{x} \\
\tilde{H}_{y}
\end{array}\right]=\left[\begin{array}{cccc}
\beta_{1} & \beta_{2} & \beta_{3} & \beta_{4} \\
\gamma_{1} & \gamma_{2} & \gamma_{3} & \gamma_{4} \\
\alpha_{1} & \alpha_{2} & \alpha_{3} & \alpha_{4} \\
1 & 1 & 1 & 1
\end{array}\right]\left[\begin{array}{cccc}
e^{\lambda, z} & 0 & 0 & 0 \\
0 & e^{-\lambda, z} & 0 & 0 \\
0 & 0 & e^{\lambda_{-} z} & 0 \\
0 & 0 & 0 & e^{-\lambda_{-} z}
\end{array}\right]\left[\begin{array}{c}
A_{1} \\
A_{2} \\
A_{3} \\
A_{4}
\end{array}\right] .
$$


The derivation of the Green's dyad of the $z$ direction bias case is the same as that of $y$ direction bias case, with the form shown in equation (181).

Equation (181) is ready to be solved by the method of moments. The only modification to be made is that the incident electric field is replaced by

$$
E^{1}+E^{r}=\left[\begin{array}{c}
E_{x}^{i}+E_{x}^{r} \\
E_{y}^{i}+E_{y}^{r}
\end{array}\right],
$$

where the $\mathbf{E}^{r}$ is the reflected electric field from the ferrite substrate by removing the patches.

According to [49], transmission matrix used in the Green's function of a generalized anisotropic medium, although rigorous analytically, it introduces a numerical breakdown, especially when the layers are electrically thick and the waves are evanescent. A variable transformation scheme introduced in [49] was used in this work and applied to the matrix in equation (140), to ensure the $4 \times 4$ transmission matrix for all the modes is nonsingular.

Equation for the reflected fields from a dielectric slab (without patches), due to a plane wave excitation, exists inliterature [45]. But for the reflected fields from the ferrite substrate with the removal of the patches, such equations are not available because of the anisotropic property of the ferrite material. Thus, an alternative way of calculation must be used here.

Rewriting equation (183) as follows 


$$
\left[\begin{array}{c}
\tilde{E}_{x} \\
\tilde{E}_{y} \\
\tilde{H}_{x} \\
\tilde{H} y
\end{array}\right]=\left[\begin{array}{cccc}
\frac{k_{0}^{2}-k_{x}^{2}}{j \omega \epsilon_{0}} & \frac{k_{0}^{2}-k_{x}^{2}}{j \omega \epsilon_{0}} & 0 & 0 \\
-\frac{k_{x} k_{y}}{j \omega \epsilon_{0}} & -\frac{k_{x} k_{y}}{j \omega \epsilon_{0}} & -\lambda_{0} & \lambda_{0} \\
0 & 0 & \frac{k_{0}^{2}-k_{x}^{2}}{j \omega \mu_{0}} & \frac{k_{0}^{2}-k_{x}^{2}}{j \omega \mu_{0}} \\
\lambda_{0} & -\lambda_{0} & -\frac{k_{x} k_{y}}{j \omega \mu_{0}} & -\frac{k_{x} k_{y}}{j \omega \mu_{0}}
\end{array}\right]
$$

Here for the waves above the slab, $G_{1}$ and $G_{3}$ correspond to the ingoing waves (see coordinate system shown in Figure 7 (a), (b)), $G_{2}$ and $G_{4}$ correspond to the outgoing waves. For the situation of a plane incident waves on a plain ferrite slab (without patches on it), the incident waves are ingoing waves in equation (193). The reflected waves are outgoing waves in equation (193). Thus equation (163) can be written as

$$
\tilde{\boldsymbol{V}}\left(0_{+}\right)=\left[\begin{array}{c}
\tilde{E}_{x}^{i}+\tilde{E}_{x}^{r} \\
\tilde{E}_{y}^{i}+\tilde{E}_{y}^{r} \\
\tilde{H}_{x}^{i}+\tilde{H}_{x}^{r} \\
\tilde{H}_{y}^{i}+\tilde{H}_{y}^{r}
\end{array}\right]=\left[\begin{array}{llll}
M_{11} & M_{12} & M_{13} & M_{14} \\
M_{21} & M_{22} & M_{23} & M_{24} \\
M_{31} & M_{32} & M_{33} & M_{34} \\
M_{41} & M_{42} & M_{43} & M_{44}
\end{array}\right]\left[\begin{array}{c}
G_{1} \\
G_{2} \\
G_{3} \\
G_{4}
\end{array}\right] .
$$

And $\tilde{\boldsymbol{V}}\left(0_{-}\right)$has the same form as in equation (179). Using the boundary condition

$$
\tilde{V}\left(0_{+}\right)-\tilde{V}\left(0_{-}\right)=0,
$$


the following expression is obtained

$$
\tilde{\boldsymbol{M}}\left[\begin{array}{c}
G_{1} \\
G_{2} \\
G_{3} \\
G_{4}
\end{array}\right]-\tilde{\boldsymbol{T}}(d) \tilde{\boldsymbol{M}} \boldsymbol{X}(-d)\left[\begin{array}{c}
G_{1}^{\prime} \\
0 \\
G_{3}^{\prime} \\
0
\end{array}\right]=0
$$

Since the incident field is known as

$$
\begin{aligned}
& E_{x}^{i}=E_{x 0} e^{-j\left(k_{x 0} x+k_{y 0} y\right)}, \\
& E_{y}^{i}=E_{y 0} e^{-j\left(k_{x 0} x+k_{y 0} y\right)},
\end{aligned}
$$

where $\mathrm{k}_{\mathrm{x} 0}$ and $\mathrm{k}_{\mathrm{y} 0}$ are $(0,0)$ Floquet modes, the Fourier transform of the incident field is linearly expressed by delta function, that is

$$
\begin{aligned}
& \tilde{E}_{x}^{i}=E_{x 0} \delta\left(k_{x 0}+k_{x}, k_{y 0}+k_{y}\right), \\
& \tilde{E}_{y}^{i}=E_{y 0} \delta\left(k_{x 0}+k_{x}, k_{y 0}+k_{y}\right) .
\end{aligned}
$$

Therefore, $G_{1}$ and $G_{3}$ in equation (192) can be expressed by the Fourier transformed incident field by equations (199) and (200). Equation (196) can be modified as

$$
\tilde{\boldsymbol{\mu}}\left[\begin{array}{c}
0 \\
G_{2} \\
0 \\
G_{4}
\end{array}\right]-\tilde{\boldsymbol{T}}(d) \tilde{\boldsymbol{M}} \boldsymbol{\boldsymbol { X }}(-d)\left[\begin{array}{c}
G_{1}^{\prime} \\
0 \\
G_{3}^{\prime} \\
0
\end{array}\right]=\left[\begin{array}{c}
C_{1}\left(\delta\left(k_{x 0}+k_{x}, k_{y 0}+k_{y}\right)\right) \\
C_{2}\left(\delta\left(k_{x 0}+k_{x}, k_{y 0}+k_{y}\right)\right) \\
C_{3}\left(\delta\left(k_{x 0}+k_{x}, k_{y 0}+k_{y}\right)\right) \\
C_{4}\left(\delta\left(k_{x 0}+k_{x}, k_{y 0}+k_{y}\right)\right)
\end{array}\right],
$$

where $C_{1}, C_{2}, C_{3}$ and $C_{4}$ are linear functions. With equation (201), $G_{2}$ and $G_{4}$ are ready 
to be solved. Thus, the Fourier transformed reflected fields from the ferrite slab are known. In the time domain, the reflected field is obtained by the following expressions

$$
\begin{aligned}
& E_{x}^{r}=\left.\tilde{E}_{x}^{r}\right|_{k_{x}=-k_{x 0}, k_{y}=-k_{y 0^{\prime}}} \\
& E_{y}^{r}=\left.\tilde{E}_{y}^{r}\right|_{k_{x}=-k_{x 0}, k_{y}=-k_{y 0}} .
\end{aligned}
$$

\subsection{POWER REFLECTION COEFFICIENT}

In the free-standing case, the frequency selective surface reflection coefficient was calculated by using the symmetry of the structure and the boundary condition on the patch. The structure of a frequency selective surface with a ferrite substrate is nonsymmetric in its normal direction ( $\mathrm{z}$ direction), thus, the method used in free-standing case can not be used here. Alternatively, the full wave power reflection coefficient is used [14]. The power reflection coefficient is as follows

$$
|R|=\frac{\operatorname{Re}\left\{\int_{\text {unit cell }} E^{s} \times H^{\cdot} \cdot 2 d \boldsymbol{s}_{\text {unit cell }}\right\}}{\operatorname{Re}\left\{\int_{\text {unit cell }} E^{1} \times H^{1 \cdot} \cdot(-\partial) d \boldsymbol{s}_{\text {unit cell }}\right\}},
$$

where the fields superscripts marked with " $\mathrm{i}$ " refer to incident fields, the fields superscripts marked with "s" refer to scattered fields from the conductor. 


\section{CHAPTER 5 \\ NUMERICAL RESULTS}

The numerical results of frequency selective surfaces on ferrite substrates are illustrated in this chapter by employing the theoretical model developed in Chapter 4 . The model derived in the previous chapter is quite flexible. It can be used to calculate either frequency selective surfaces on dielectric/ferrite substrates or free-standing surfaces. In order to verify the validity of this model, the calculated results from this model are compared with the published ones [8], [20] in this chapter. Several calculated results for the frequency selective surfaces on ferrite substrates, as functions of the applied dc bias, the thickness of the substrate, the dielectric constant of the substrate, are presented. Other characteristics are also presented and discussed.

\subsection{VERIFICATION}

The model for ferrite substrate frequency selective surfaces is valid to any dc bias, thickness and dielectric constant of the substrate. It can be easily used to calculate the frequency selective surfaces with different substrates. Since there was no previous 
published results related to the ferrite substrate frequency selective surfaces with rectangular patches, to prove the validity of this model, some special cases are selected.

The first verification is the simulation of the free-standing case. It is realized by assuming the bias of the ferrite substrate being zero and the dielectric constant $\varepsilon_{\mathrm{r}}=1.001$ $\left(\varepsilon_{r}=1\right.$ will result in a singular coefficient matrix for the method of moments), the thickness of the substrate is set to $2 . \mathrm{e}-8 \mathrm{~cm}$ (the thickness can not be zero, with the same reason as before). Basically, this situation is equivalent to the free-standing one. The unit cell of the frequency selective surfaces is with the rectangular patch as shown in Figure 8. The dimension of the unit cell is $a a=0.76 \mathrm{~cm}, b b=0.254 \mathrm{~cm}, c c=1.52 \mathrm{~cm}$, $\mathrm{dd}=1.35 \mathrm{~cm}$. The frequency response of the surface reflection coefficient is compared with C. H. Chan's result [8]. They are in very good agreement as shown in Figure 9.

The second verification is done by using dielectric substrate cases with the assumption that the bias of the ferrite substrate is zero. J. P. Montgomery in [20] presented both calculated and measured reflection coefficient for frequency selective surfaces with rectangular patches on dielectric substrates. His measurement is performed with a waveguide simulator. The experiments are conducted at a frequency $\mathrm{f}=4.156 \mathrm{GHz}$, with an incident angle of $40^{\circ}$. Three sets of results are compared. The parameters for these sets are as follows. The first set is $a a=0.901 \mathrm{~cm}, b b=0.444 \mathrm{~cm}, c c=3.58 \mathrm{~cm}$, $\mathrm{dd}=1.98 \mathrm{~cm}, \mathrm{~d}=0.0203 \mathrm{~cm}, \varepsilon_{\mathrm{r}}=2.6$. The calculated and experimented reflection coefficients from Montgomery is 0.465 and 0.495 , respectively. The calculated reflection coefficient from this model is 0.420 . The second set has the same geometry parameters as the first 


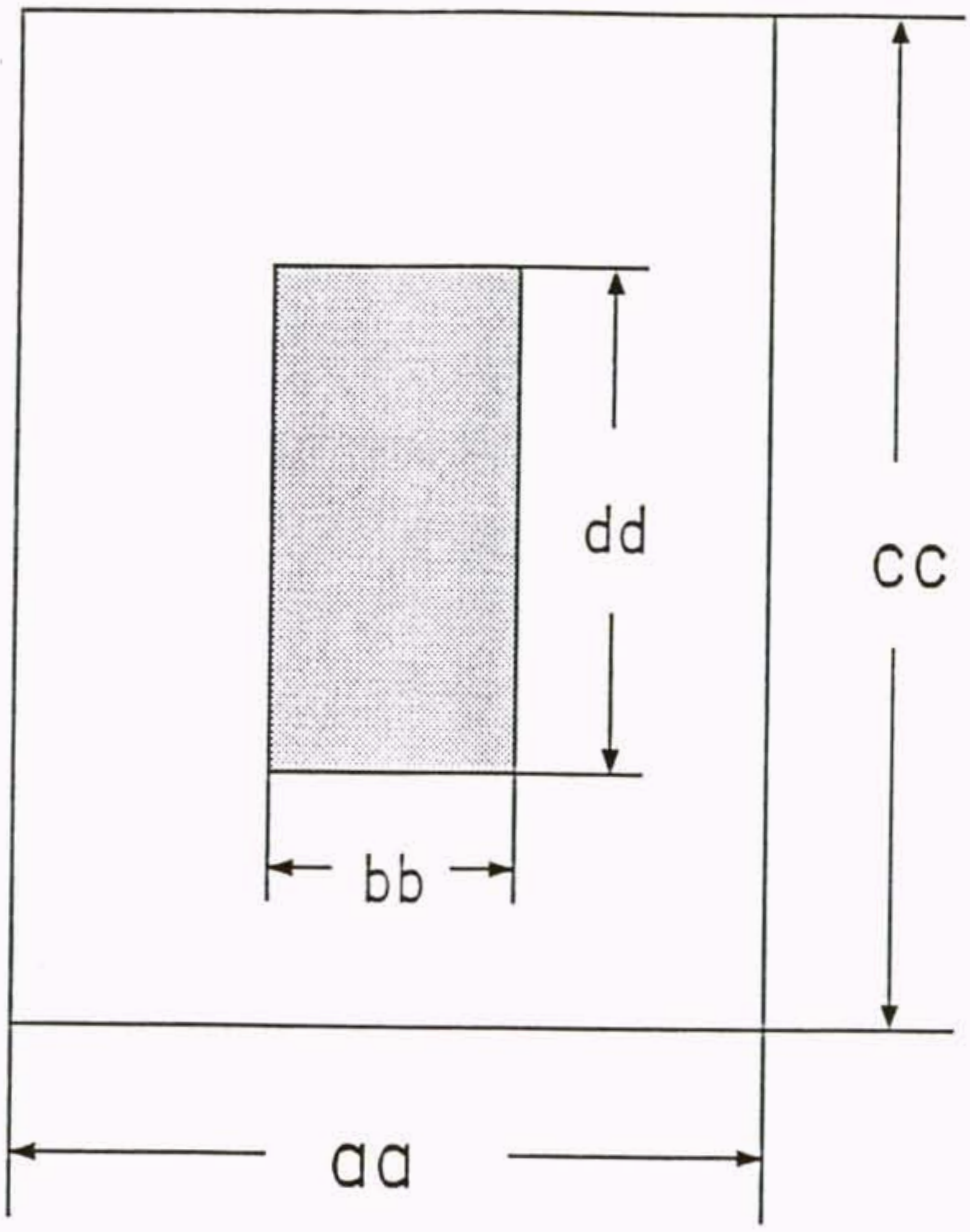

Figure 8. Geometry of a unit cell (patch) for frequency selective surface. 


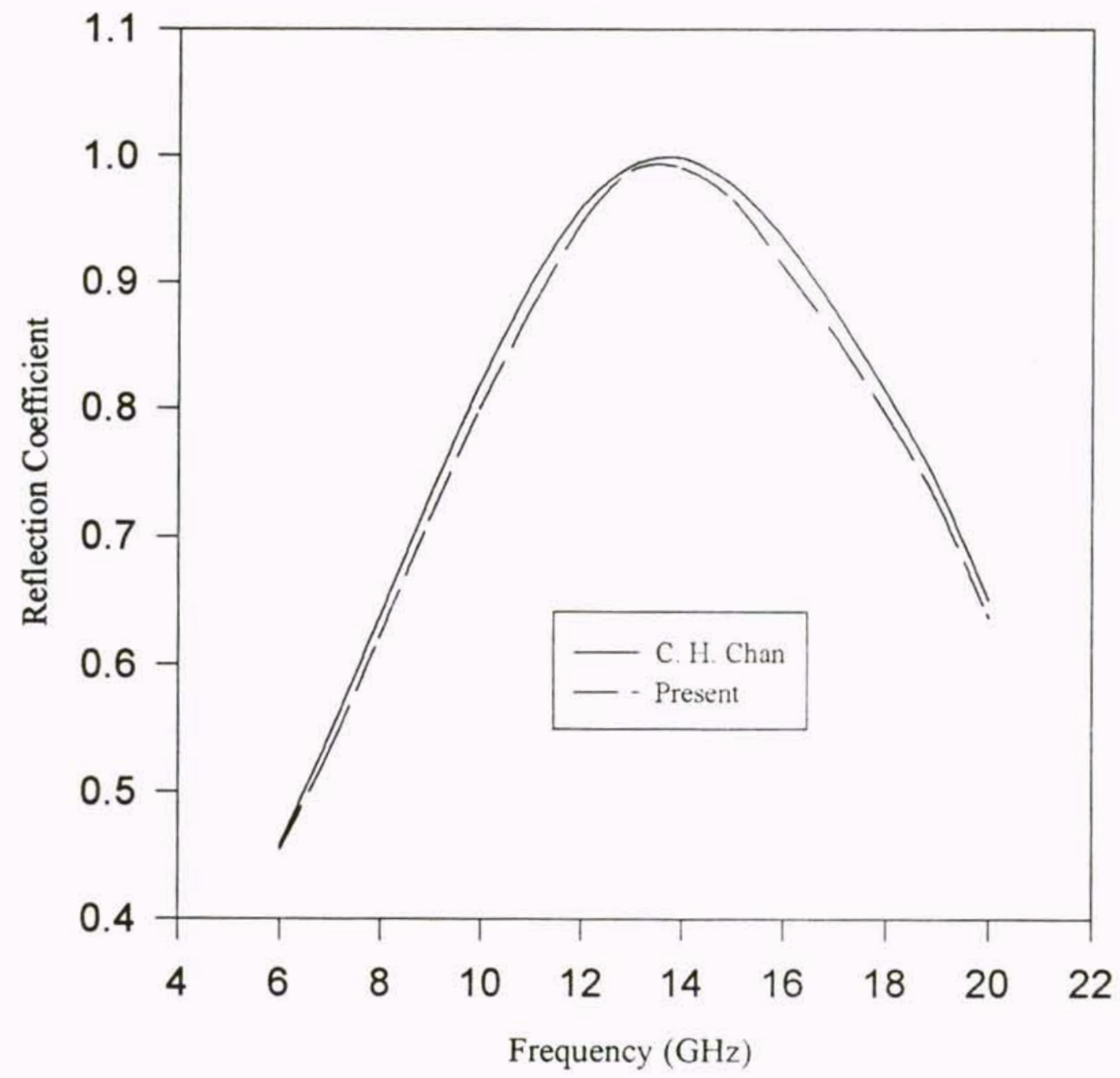

Figure 9. Comparison of free-standing case with published data [8]. 
set with the exception of $d=0.0317$. The calculated and experimented results from Montgomery is 0.615 and 0.656 , respectively. The calculated result from this model is 0.6085 . The third set is $a a=3.58 \mathrm{~cm}, b b=1.98 \mathrm{~cm}, c c=0.901 \mathrm{~cm}, d d=0.444 \mathrm{~cm}, d=0.0203 \mathrm{~cm}$, $\varepsilon_{\mathrm{r}}=2$.6. The calculated and experimented results from Montgomery is 0.099 and 0.095 , respectively. The calculated result from this model is 0.0738 .

\subsection{RESULTS FOR FREQUENCY SELECTIVE SURFACES ON FERRITE SUBSTRATES}

The computer program of this theoretical model is written by FORTRAN. It is suitable for ferrite substrate cases, including different biases (in-plane bias or normal bias), different incident wave polarizations (TE mode or TM mode), and different incident angles. After some adjustment of the input data, it can also be used to analyze frequency selective surfaces on dielectric substrates or free-standing case.

All the calculations are based upon substrates made from yttrium iron garnet (YIG), with saturation magnetization $4 \pi \mathrm{M}_{\mathrm{s}}=1780 \mathrm{G}$. The bias field is changed from 2000 Oe to 5000 Oe. Two different dielectric constants $\left(\varepsilon_{\mathrm{t}}=12.8\right.$ and $\left.\varepsilon_{\mathrm{r}}=15\right)$ for the ferrite substrate are chosen. For some cases, different thicknesses of the substrate (from $d=0.02 \mathrm{~cm}$ to $\mathrm{d}=0.08 \mathrm{~cm}$ ) are considered. The dimension of unit cell for all TE incident cases is $\mathrm{a} a=0.76 \mathrm{~cm}, \mathrm{bb}=0.254 \mathrm{~cm}, \mathrm{cc}=1.52 \mathrm{~cm}, \mathrm{dd}=1.35 \mathrm{~cm}$, except for the TM incident cases which 
is $a a=1.52 \mathrm{~cm}, b b=0.76 \mathrm{~cm}, c c=1.52 \mathrm{~cm}, \mathrm{dd}=1.35 \mathrm{~cm}$.

The calculation program of the frequency selective surfaces on ferrite substrates is performed on a Sun work station. The unit cell of the frequency selective surfaces is discretized into a $16 \times 16$ grid with 94 unknowns for TE incident cases. The Floquet modes are truncated between $\mathbf{- 3 2}$ and 32 . This number of modes yields accurate enough results and needs no higher number of modes. To get the power reflection coefficient for one frequency point, the total running time is about 1.5 hour for 94 unknowns on Sun Sparc Station 1.

Figures 10 to 13 show the power reflection coefficient frequency responses with the different dc biases $\mathrm{H}_{0}$, at $\varepsilon_{\mathrm{r}}=12.8$. Figures 14 and 15 depict the power reflection coefficient frequency responses for the different dc biases $H_{0}$, with $\varepsilon_{\mathrm{r}}=15$. In Figure 16, the $\mathrm{z}$-bias cases are presented. Figures 17 to 20 illustrate the power reflection coefficient frequency responses with the different thicknesses $d$, for $\varepsilon_{\mathrm{r}}=12.8$. Figures 21 and 22 present the power reflection coefficient frequency responses with the different thicknesses d, at $\varepsilon_{\mathrm{r}}=15$. Figures 23 to 26 show the power reflection coefficient frequency responses with the different dielectric constants $\varepsilon_{\mathrm{r}}$. Figure 27 shows the comparison of calculation results using diferent Floquet modes. 


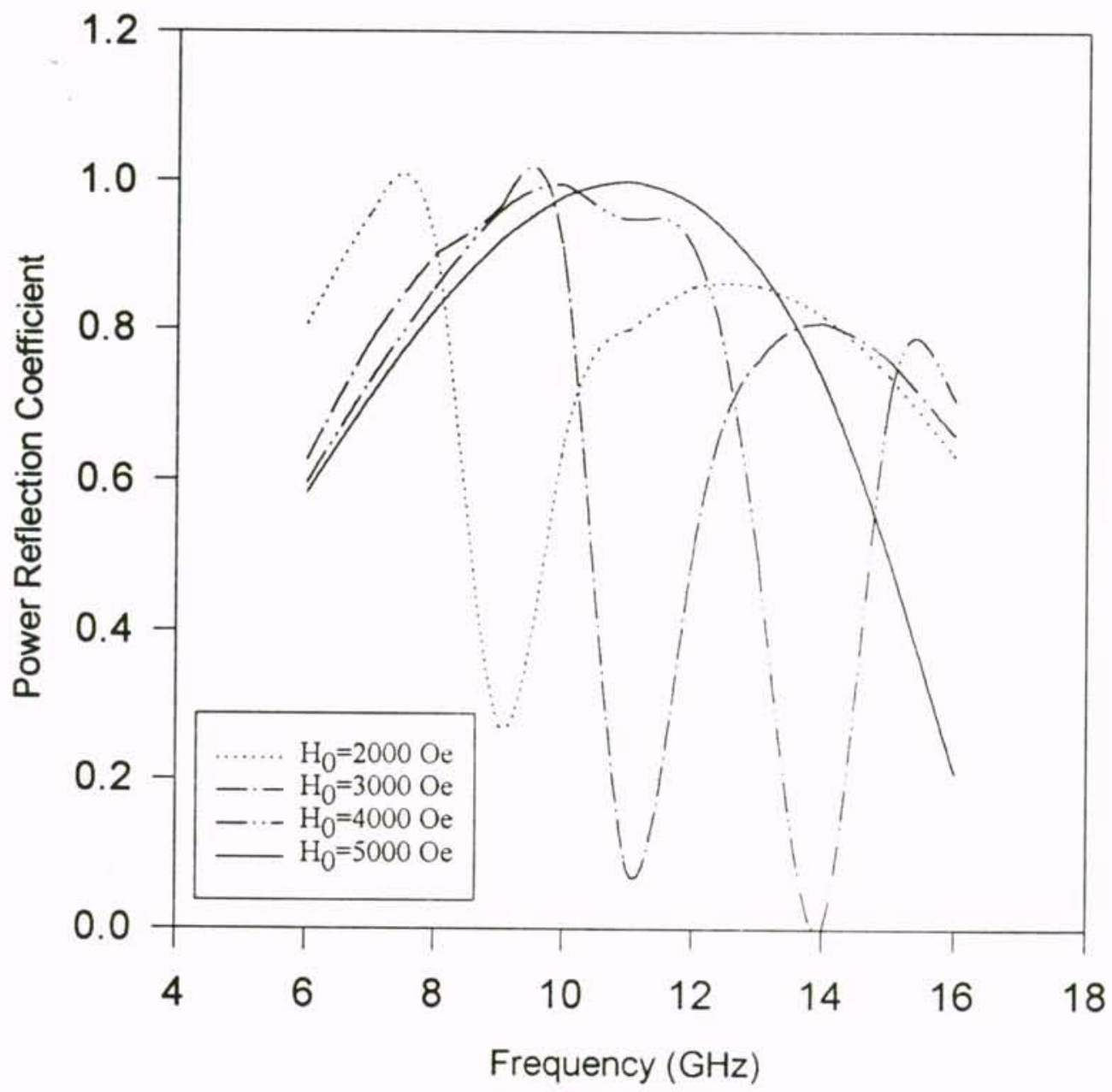

Figure 10. TE normal incidence, $y$-bias, $4 \pi \mathrm{M}_{\mathrm{s}}=1780 \mathrm{G}, \varepsilon_{\mathrm{r}}=12.8, \mathrm{~d}=0.02 \mathrm{~cm}$, with different dc biases. $a \mathrm{a}=0.76 \mathrm{~cm}, \mathrm{bb}=0.254 \mathrm{~cm}, \mathrm{cc}=1.52 \mathrm{~cm}, \mathrm{dd}=1.35 \mathrm{~cm}$. 


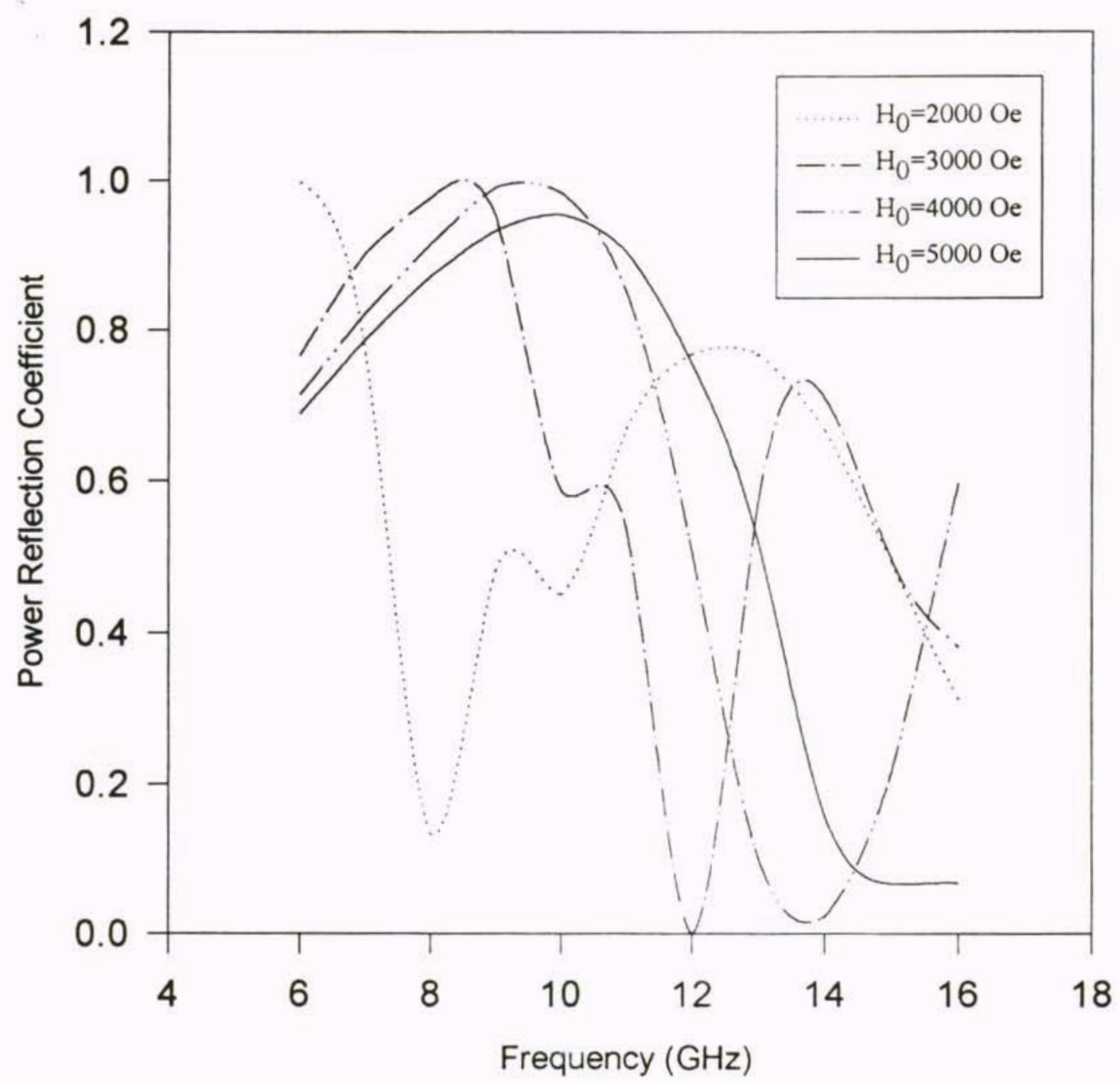

Figure 11. TE normal incidence, $y$-bias, $4 \pi \mathrm{M}_{\mathrm{s}}=1780 \mathrm{G}, \varepsilon_{\mathrm{r}}=12.8, \mathrm{~d}=0.04 \mathrm{~cm}$, with different dc biases. $a a=0.76 \mathrm{~cm}, b b=0.254 \mathrm{~cm}, c c=1.52 \mathrm{~cm}, d d=1.35 \mathrm{~cm}$. 


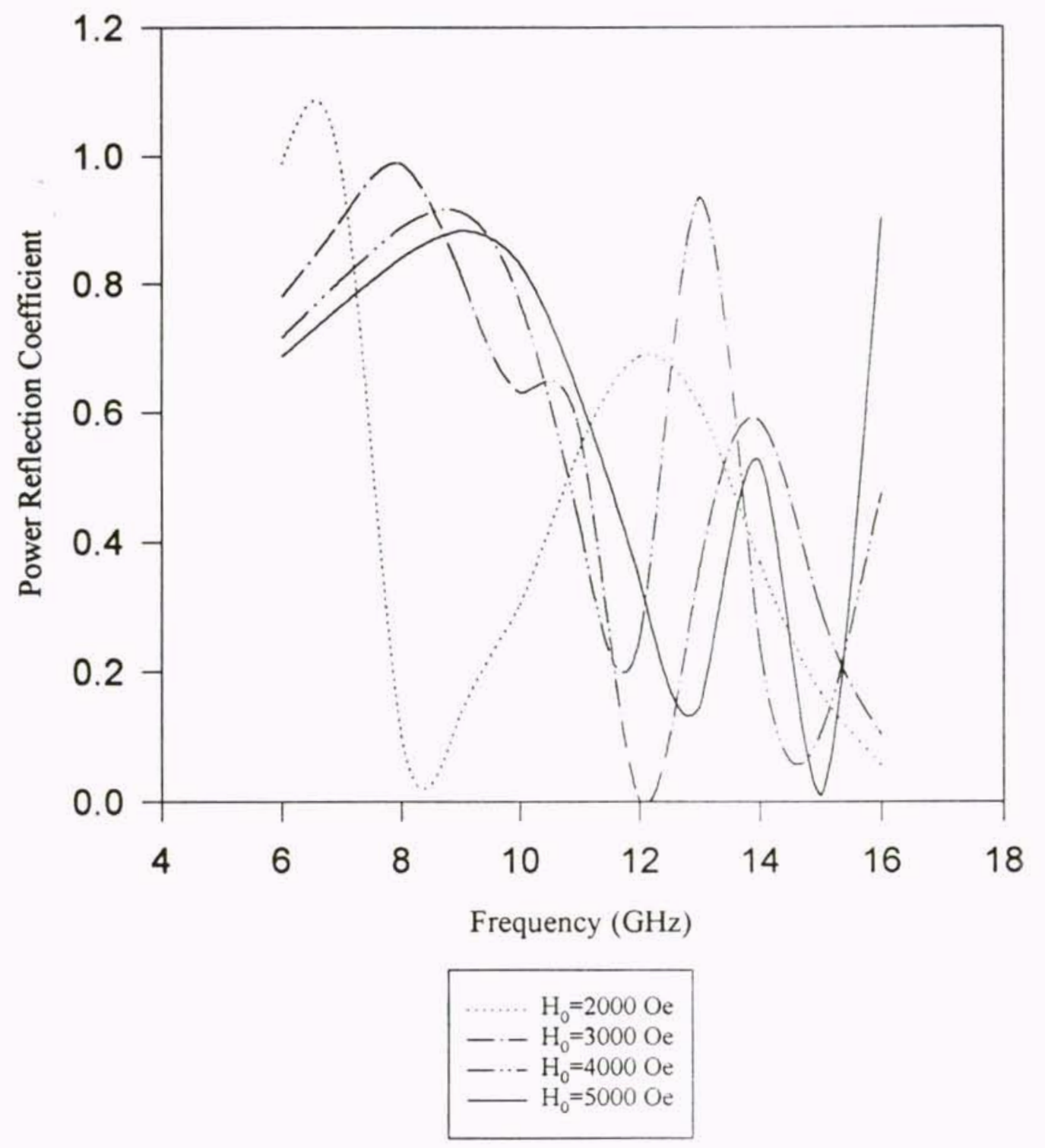

Figure 12. TE normal incidence, $y$-bias, $4 \pi \mathrm{M}_{\mathrm{s}}=1780 \mathrm{G}, \varepsilon_{\mathrm{r}}=12.8, \mathrm{~d}=0.06 \mathrm{~cm}$, with different dc biases. $a a=0.76 \mathrm{~cm}, b b=0.254 \mathrm{~cm}, c c=1.52 \mathrm{~cm}, \mathrm{dd}=1.35 \mathrm{~cm}$. 


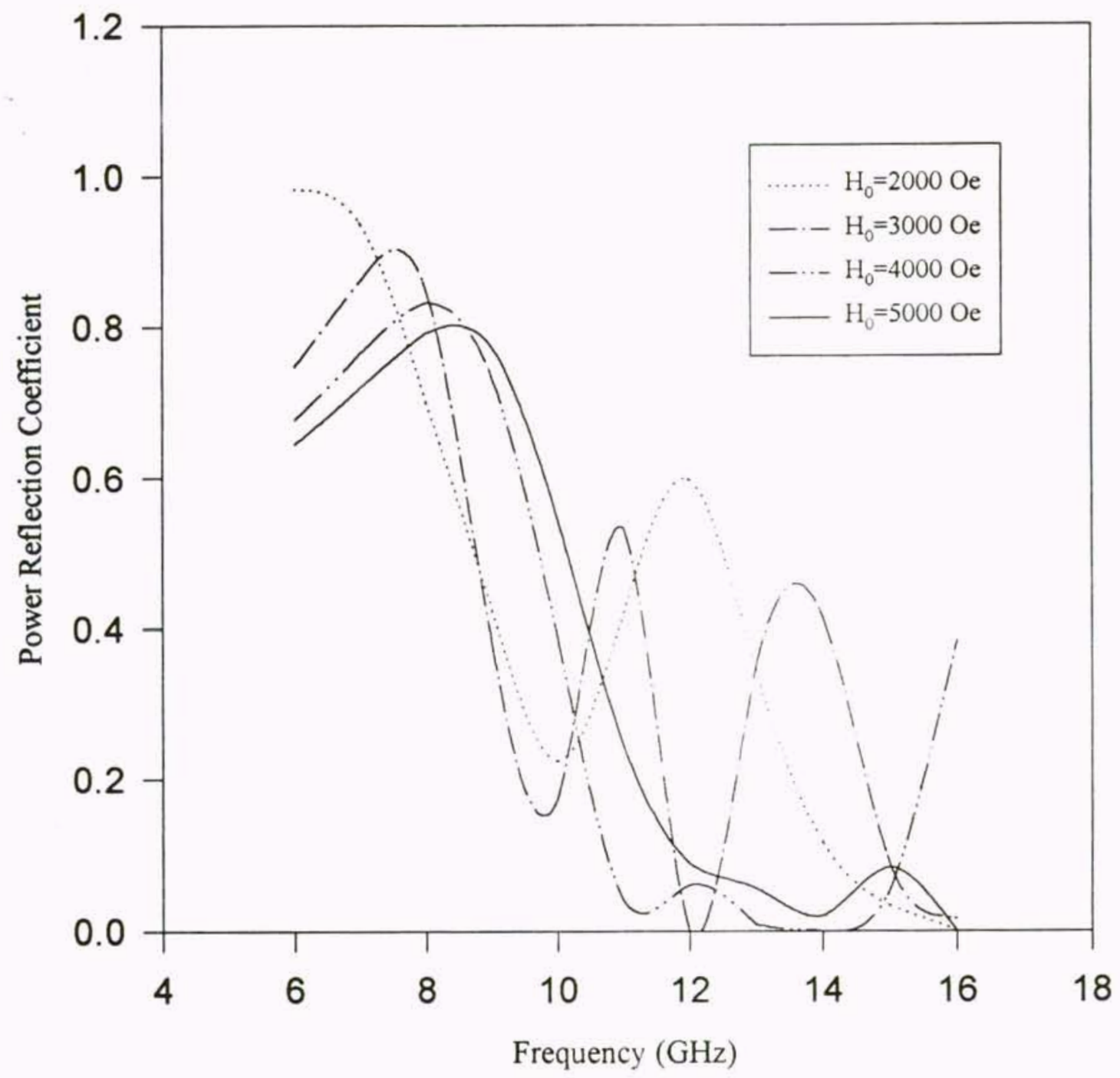

Figure 13. TE normal incidence, $y$-bias, $4 \pi \mathrm{M}_{\mathrm{s}}=1780 \mathrm{G}, \varepsilon_{\mathrm{r}}=12.8, \mathrm{~d}=0.08 \mathrm{~cm}$, with different dc biases. $a \mathrm{a}=0.76 \mathrm{~cm}, \mathrm{bb}=0.254 \mathrm{~cm}, \mathrm{cc}=1.52 \mathrm{~cm}, \mathrm{dd}=1.35 \mathrm{~cm}$. 


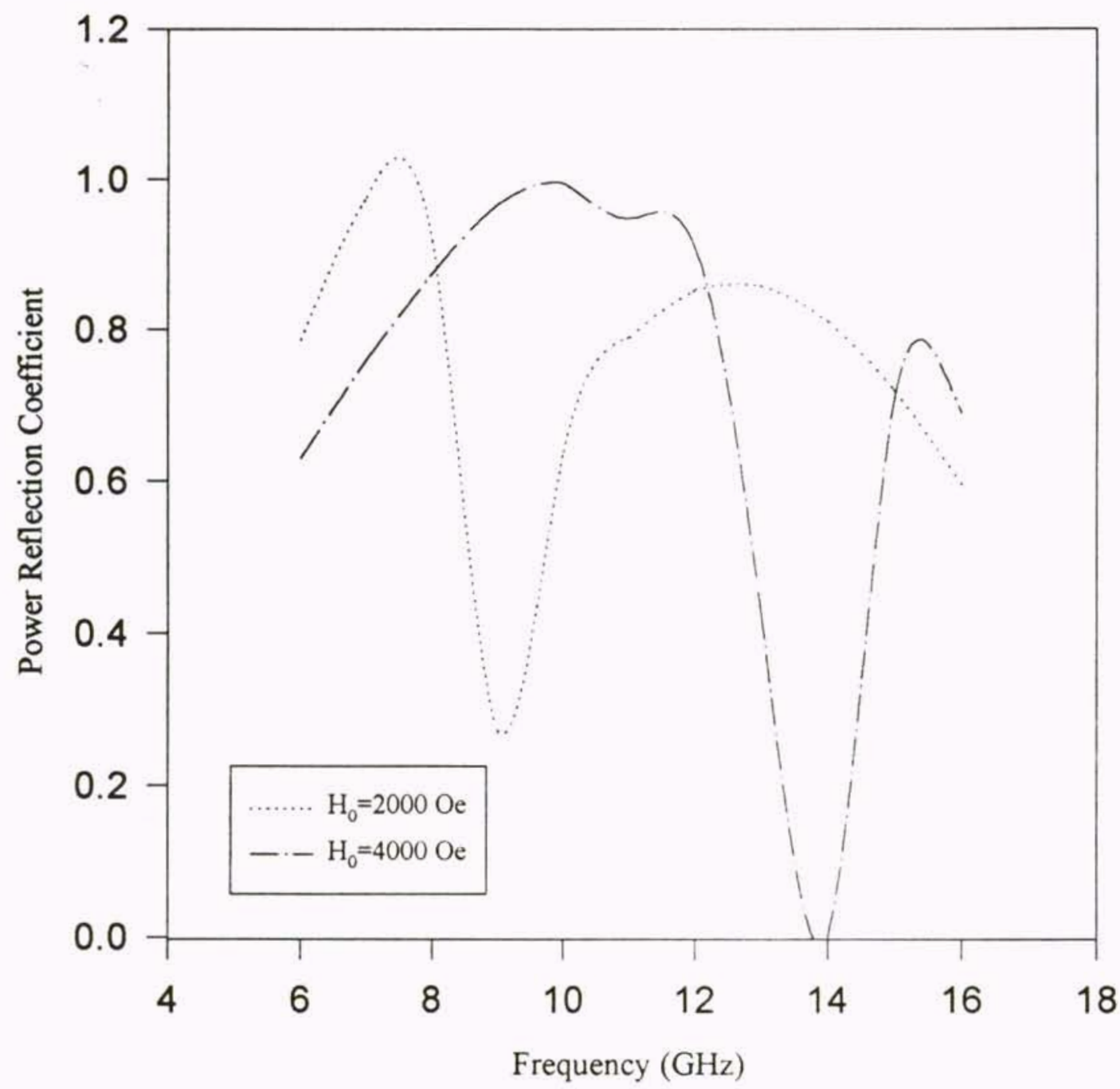

Figure 14. TE normal incidence, $y$-bias, $4 \pi \mathrm{M}_{\mathrm{s}}=1780 \mathrm{G}, \varepsilon_{\mathrm{r}}=15, \mathrm{~d}=0.02 \mathrm{~cm}$, with different $\mathrm{dc}$ biases. $\mathrm{aa}=0.76 \mathrm{~cm}, \mathrm{bb}=0.254 \mathrm{~cm}, \mathrm{cc}=1.52 \mathrm{~cm}, \mathrm{dd}=1.35 \mathrm{~cm}$. 


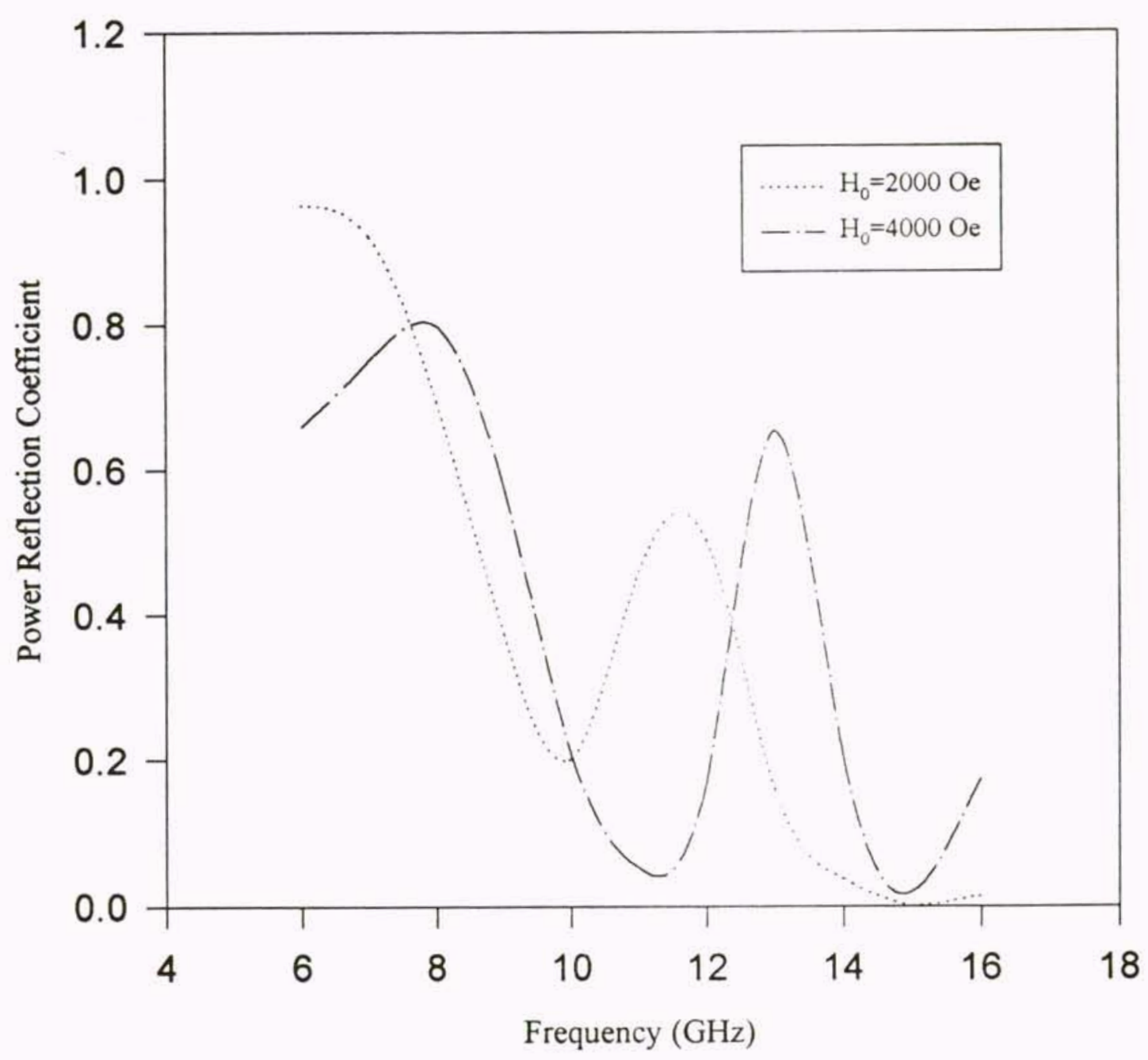

Figure 15. TE normal incidence, $y$-bias, $4 \pi \mathrm{M}_{\mathrm{s}}=1780 \mathrm{G}, \varepsilon_{\mathrm{r}}=15, \mathrm{~d}=0.08 \mathrm{~cm}$, with different dc biases. $a a=0.76 \mathrm{~cm}, b b=0.254 \mathrm{~cm}, c c=1.52 \mathrm{~cm}, d d=1.35 \mathrm{~cm}$. 


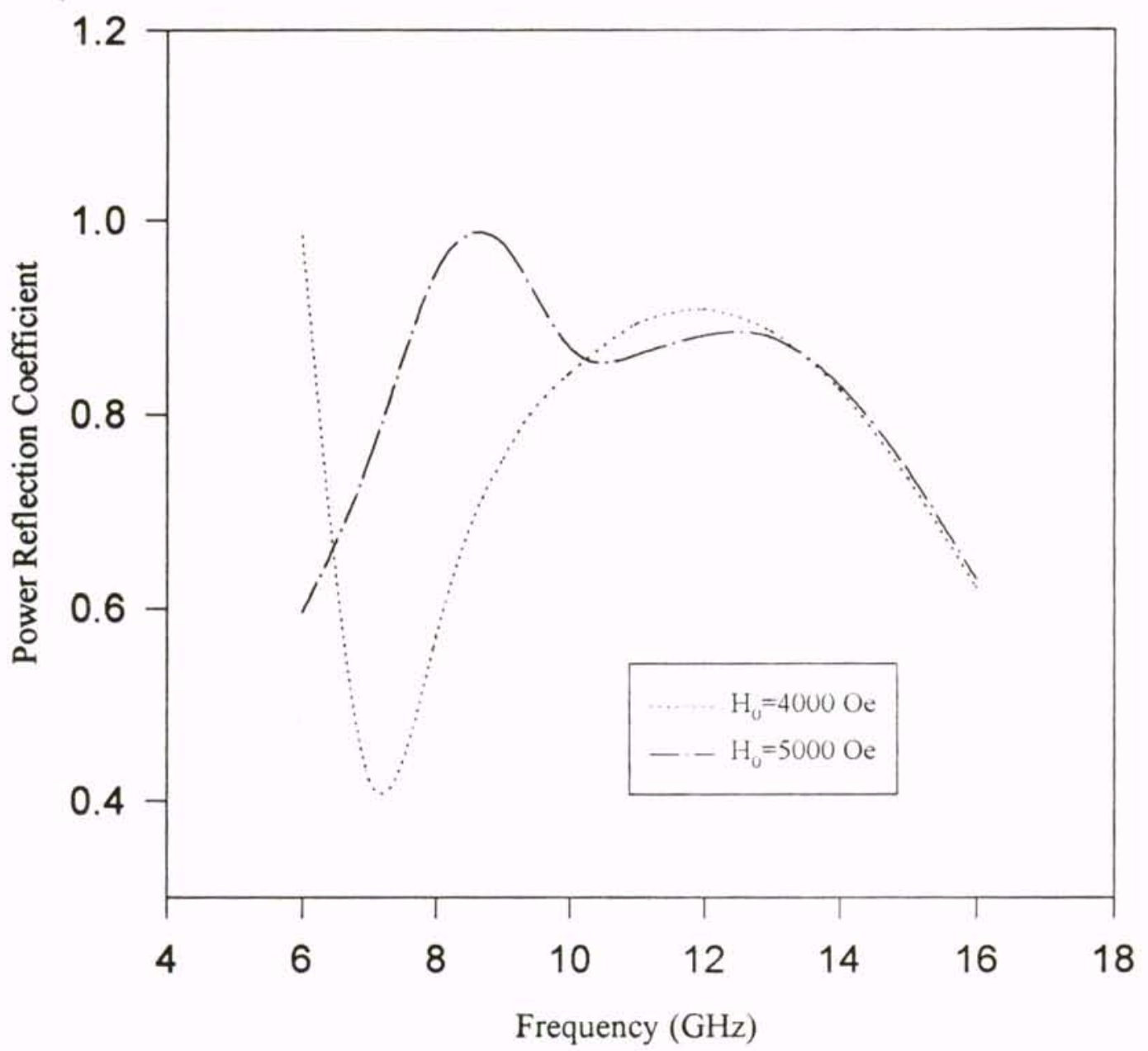

Figure 16. TE normal incidence, $\mathrm{z}$-bias, $4 \pi \mathrm{M}_{\mathrm{s}}=1780 \mathrm{G}, \varepsilon_{\mathrm{r}}=12.8, \mathrm{~d}=0.02 \mathrm{~cm}$, with different dc biases. $a a=0.76 \mathrm{~cm}, b b=0.254 \mathrm{~cm}, c c=1.52 \mathrm{~cm}, d d=1.35 \mathrm{~cm}$. 


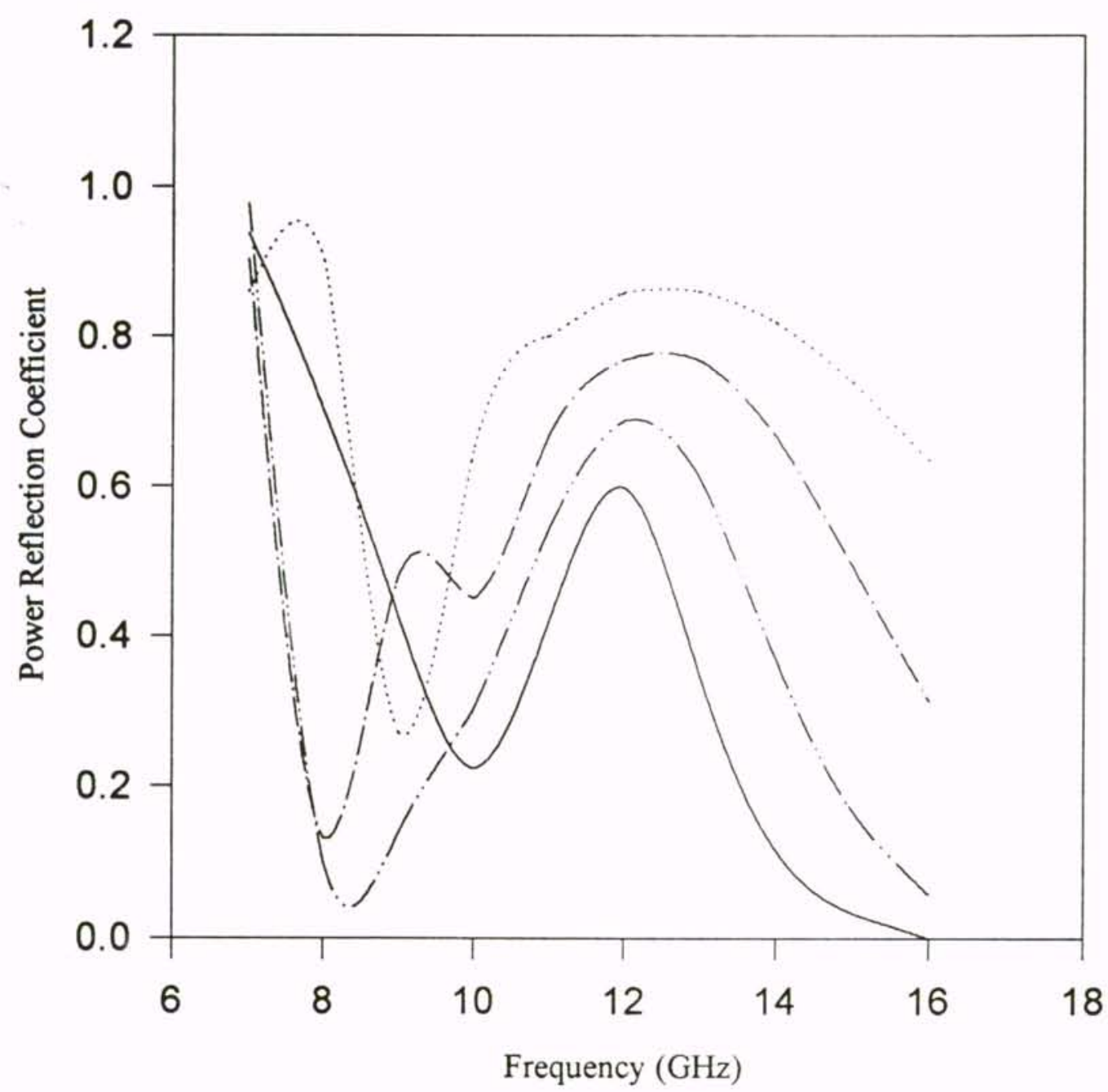

Figure 17. TE normal incidence, $y$-bias, $4 \pi \mathrm{M}_{\mathrm{s}}=1780 \mathrm{G}, \varepsilon_{\mathrm{r}}=12.8, \mathrm{H}_{0}=20000 \mathrm{e}$, with different substrate thicknesses. $a a=0.76 \mathrm{~cm}, b b=0.254 \mathrm{~cm}, c c=1.52 \mathrm{~cm}$, $\mathrm{dd}=1.35 \mathrm{~cm}$. 


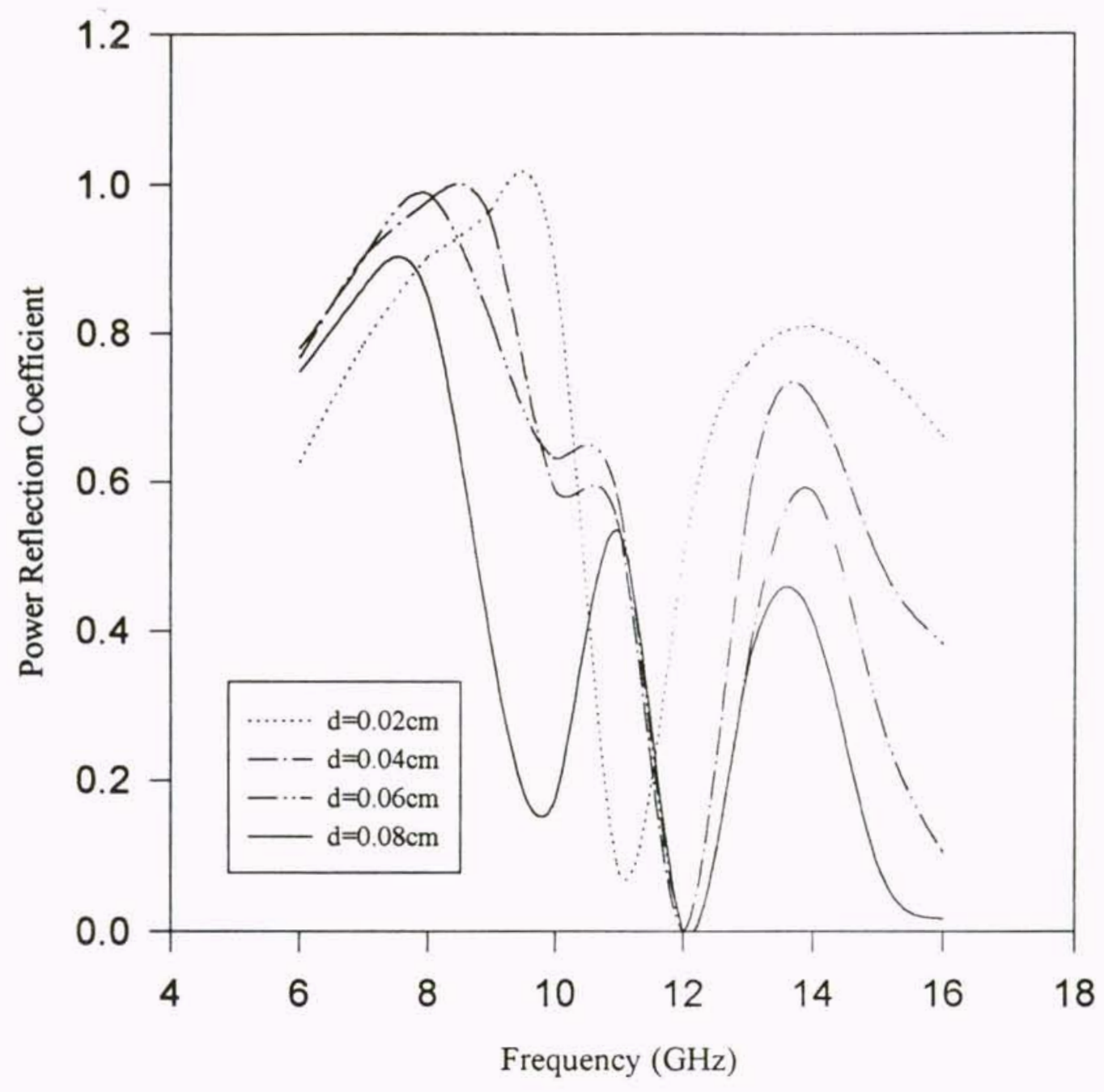

Figure 18. TE normal incidence, $y$-bias, $4 \pi \mathrm{M}_{\mathrm{s}}=1780 \mathrm{G}, \varepsilon_{\mathrm{r}}=12.8, \mathrm{H}_{0}=30000 \mathrm{e}$, with different substrate thicknesses. $a a=0.76 \mathrm{~cm}, b b=0.254 \mathrm{~cm}, c c=1.52 \mathrm{~cm}$, $\mathrm{dd}=1.35 \mathrm{~cm}$. 


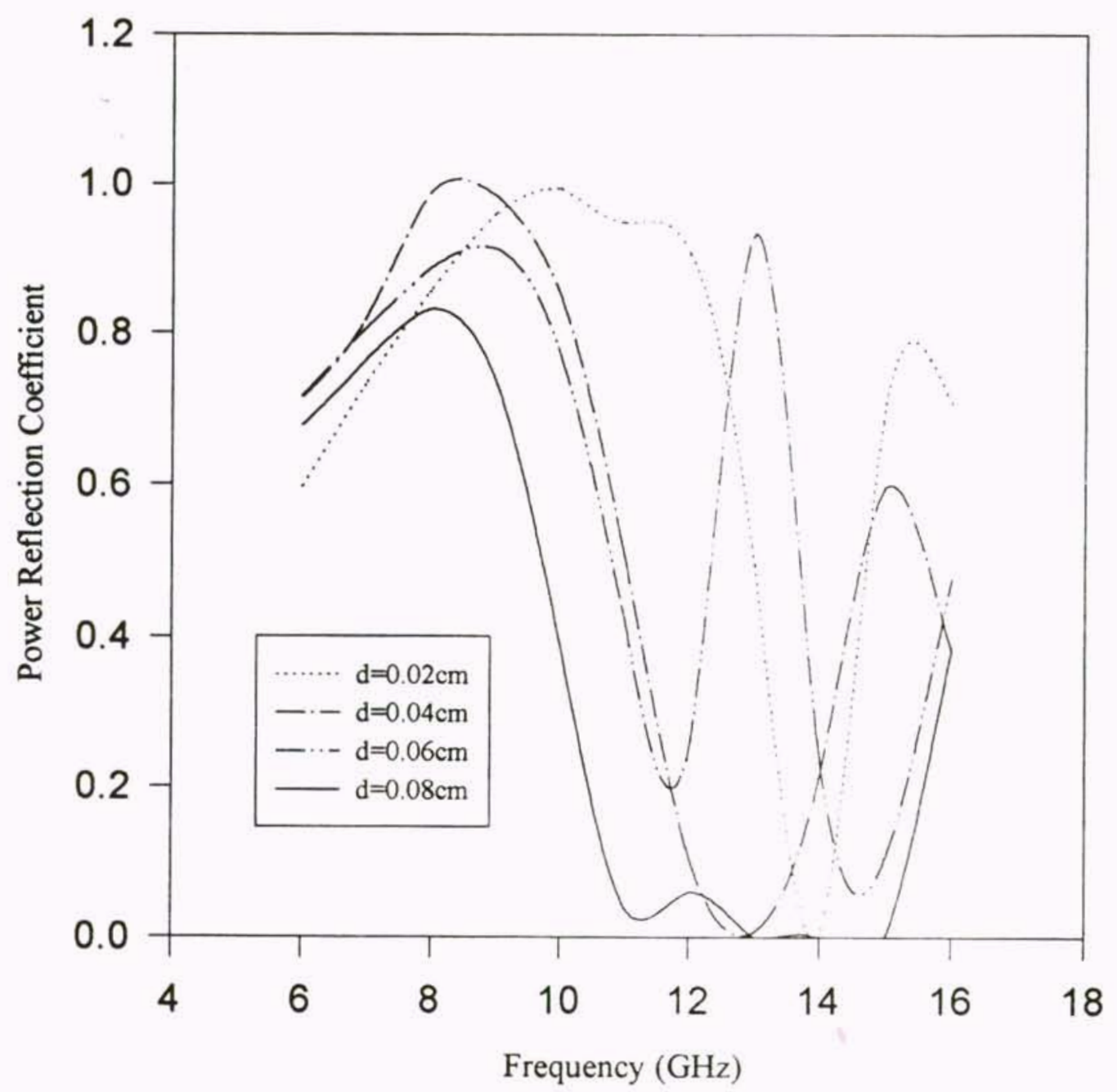

Figure 19. TE normal incidence, $y$-bias, $4 \pi \mathrm{M}_{\mathrm{s}}=1780 \mathrm{G}, \varepsilon_{\mathrm{r}}=12.8, \mathrm{H}_{0}=40000$, with different substrate thicknesses. $a \mathrm{a}=0.76 \mathrm{~cm}, \mathrm{bb}=0.254 \mathrm{~cm}, \mathrm{cc}=1.52 \mathrm{~cm}$, $\mathrm{dd}=1.35 \mathrm{~cm}$. 


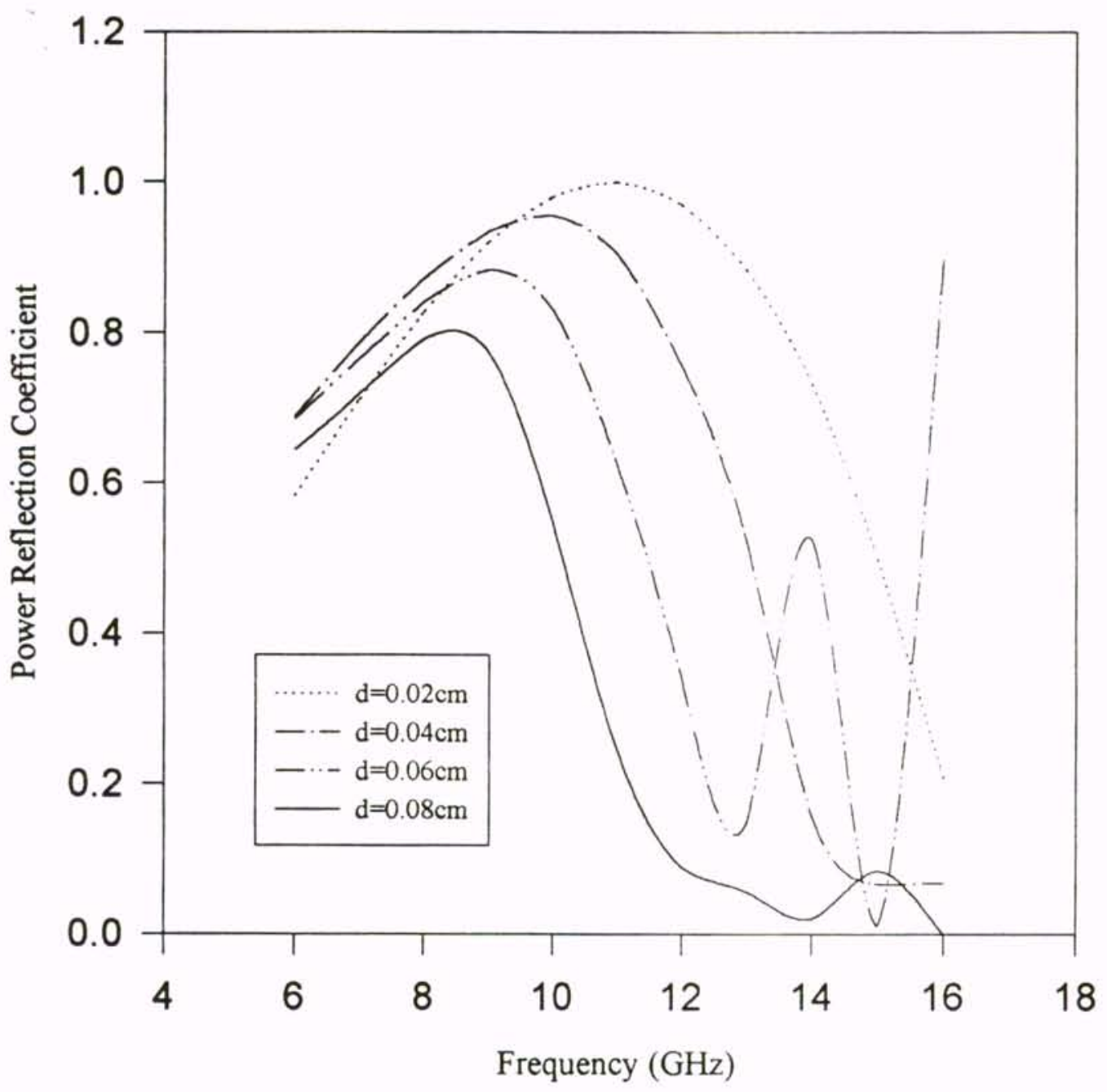

Figure 20. TE normal incidence, $y$-bias, $4 \pi \mathrm{M}_{\mathrm{s}}=1780 \mathrm{G}, \varepsilon_{\mathrm{r}}=12.8, \mathrm{H}_{0}=50000 \mathrm{e}$, with different substrate thicknesses. $a \mathrm{a}=0.76 \mathrm{~cm}, \mathrm{bb}=0.254 \mathrm{~cm}, \mathrm{cc}=1.52 \mathrm{~cm}$, $\mathrm{dd}=1.35 \mathrm{~cm}$. 


\subsection{DISCUSSION}

The effect of a ferrite substrate on frequency selective surface seems similar to a dielectric substrate, yet it is different due to some special properties of ferrite materials. The ferrite substrate can support surface waves and, under certain conditions, a magnetostatic volume wave. The magnetization vector of a ferrite material precesses about the DC field, $\mathrm{H}_{0}$, when it is put in a bias field. This precession strongly affects the RHCP modes (see Chapter 3) when the wave propagating parallel to the bias field and E modes when normal to the bias field, resulting in the two very different modes (RHCP and LHCP, or, E and $\mathrm{O}$ ). The deviation of the two modes depends not only on the bias field but also the frequency of the RF signal.

The most apparent behavior that ferrite substrate frequency selective surface possesses is the tunability of the resonant frequency. For the $y$ direction (in-plane) bias, with an increase of the bias field, the resonant frequency also increases, regardless of the thickness or dielectric constant. The tendency of the resonance shift is consistent with that of the ferrite material itself (see equation (78) in Chapter 3). Apparently, the shift of the ferrite material affects the resonance of the frequency selective surface, which relates to the geometry of the patch, the thickness and the dielectric constant of the substrate. The resonance for normally biased cases is lower than zero bias one for the same substrate thickness. From Figures 10 to 13 , it can be seen that there is about a 3 $\mathrm{GHz}$ shift with the change of the bias field. It is in agreement with the experimental 


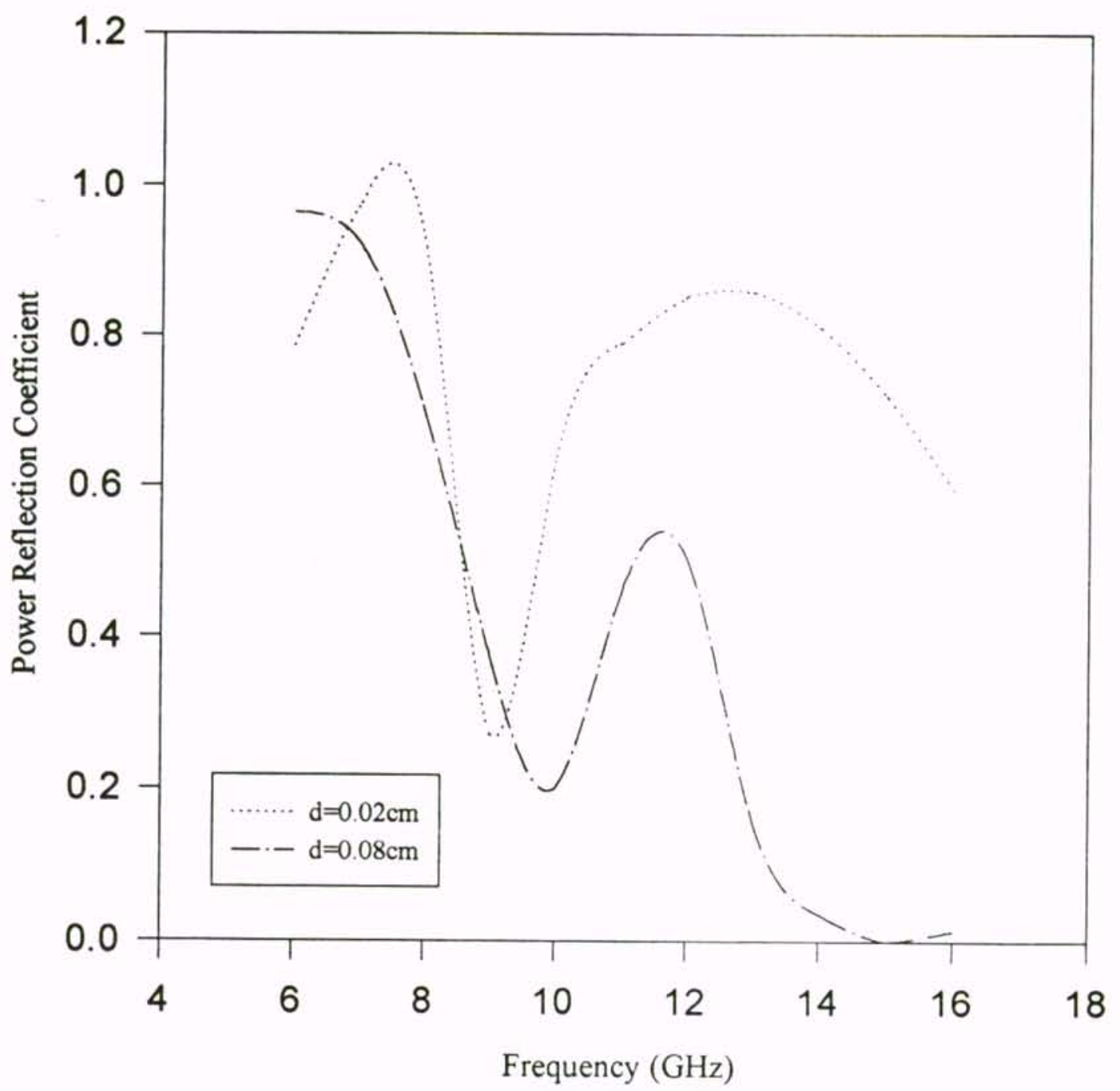

Figure 21. TE normal incidence, $\mathrm{y}$-bias, $4 \pi \mathrm{M}_{\mathrm{s}}=1780 \mathrm{G}, \varepsilon_{\mathrm{r}}=15, \mathrm{H}_{0}=2000 \mathrm{Oe}$, with different substrate thicknesses. $a \mathrm{a}=0.76 \mathrm{~cm}, \mathrm{bb}=0.254 \mathrm{~cm}, \mathrm{cc}=1.52 \mathrm{~cm}$, $\mathrm{dd}=1.35 \mathrm{~cm}$. 


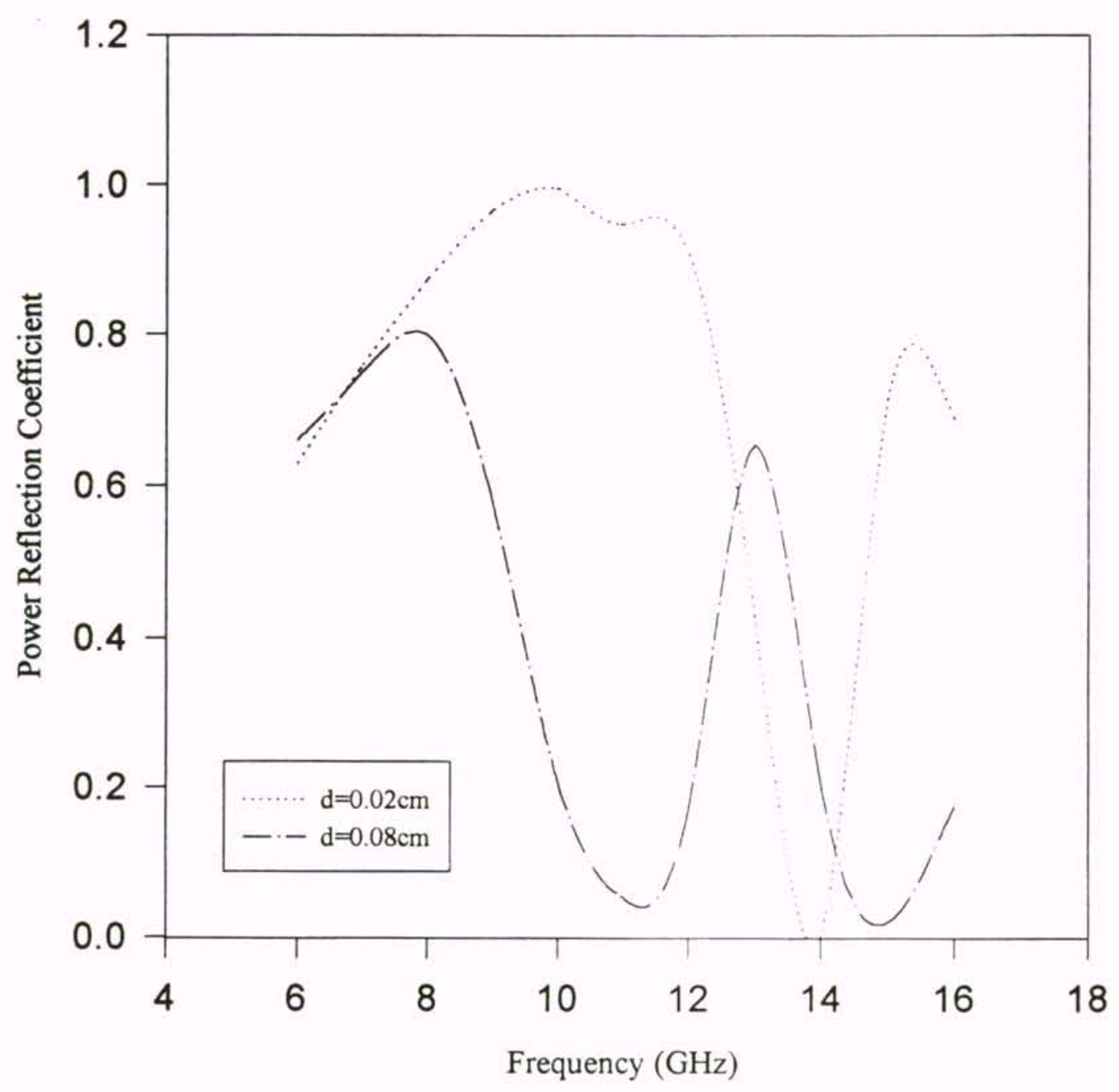

Figure 22. TE normal incidence, $\mathrm{y}$-bias, $4 \pi \mathrm{M}_{\mathrm{s}}=1780 \mathrm{G}, \varepsilon_{\mathrm{r}}=15, \mathrm{H}_{0}=40000 \mathrm{e}$, with different substrate thicknesses. $a a=0.76 \mathrm{~cm}, b b=0.254 \mathrm{~cm}, c c=1.52 \mathrm{~cm}$, $\mathrm{dd}=1.35 \mathrm{~cm}$. 


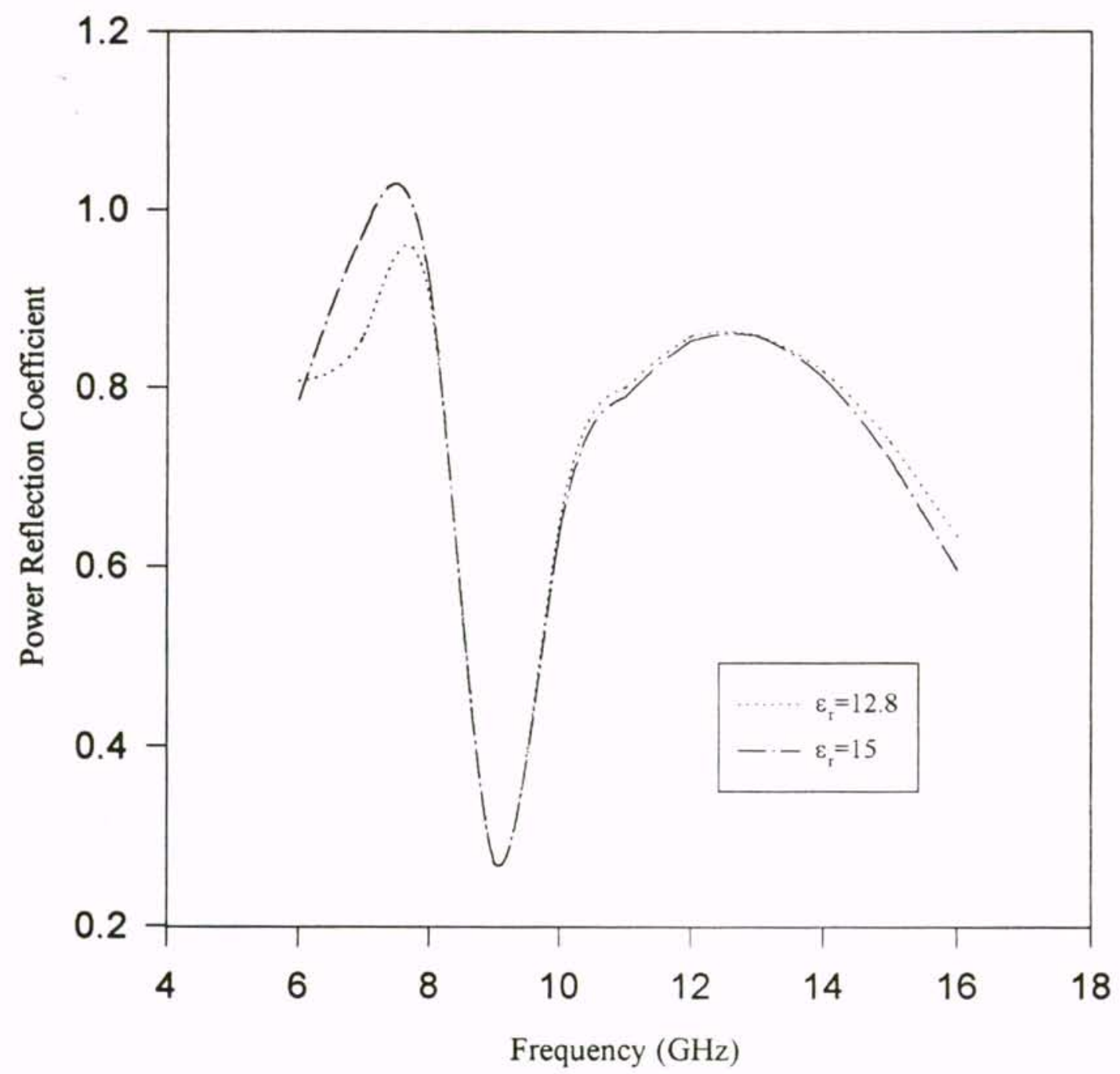

Figure 23. TE normal incidence, $y$-bias, $4 \pi \mathrm{M}_{\mathrm{s}}=1780 \mathrm{G}, \mathrm{d}=0.02 \mathrm{~cm}, \mathrm{H}_{0}=20000 \mathrm{e}$, with different dielectric constants. $a \mathrm{a}=0.76 \mathrm{~cm}, \mathrm{bb}=0.254 \mathrm{~cm}, \mathrm{cc}=1.52 \mathrm{~cm}$, $\mathrm{dd}=1.35 \mathrm{~cm}$. 


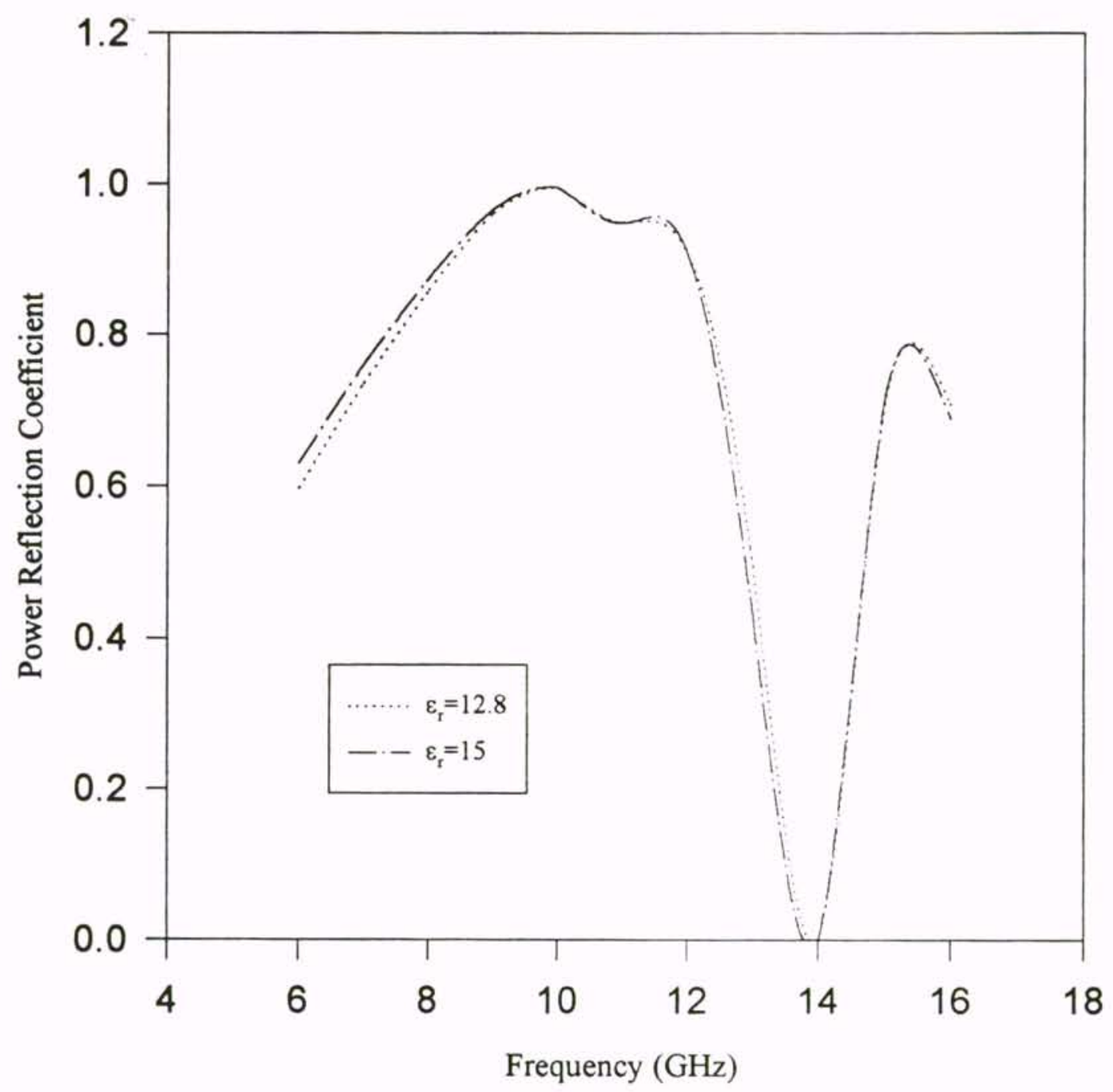

Figure 24. TE normal incidence, $y$-bias, $4 \pi \mathrm{M}_{\mathrm{s}}=1780 \mathrm{G}, \mathrm{d}=0.02 \mathrm{~cm}, \mathrm{H}_{0}=4000 \mathrm{Oe}$, with different dielectric constants. $a a=0.76 \mathrm{~cm}, b b=0.254 \mathrm{~cm}, c c=1.52 \mathrm{~cm}$, $\mathrm{dd}=1.35 \mathrm{~cm}$. 


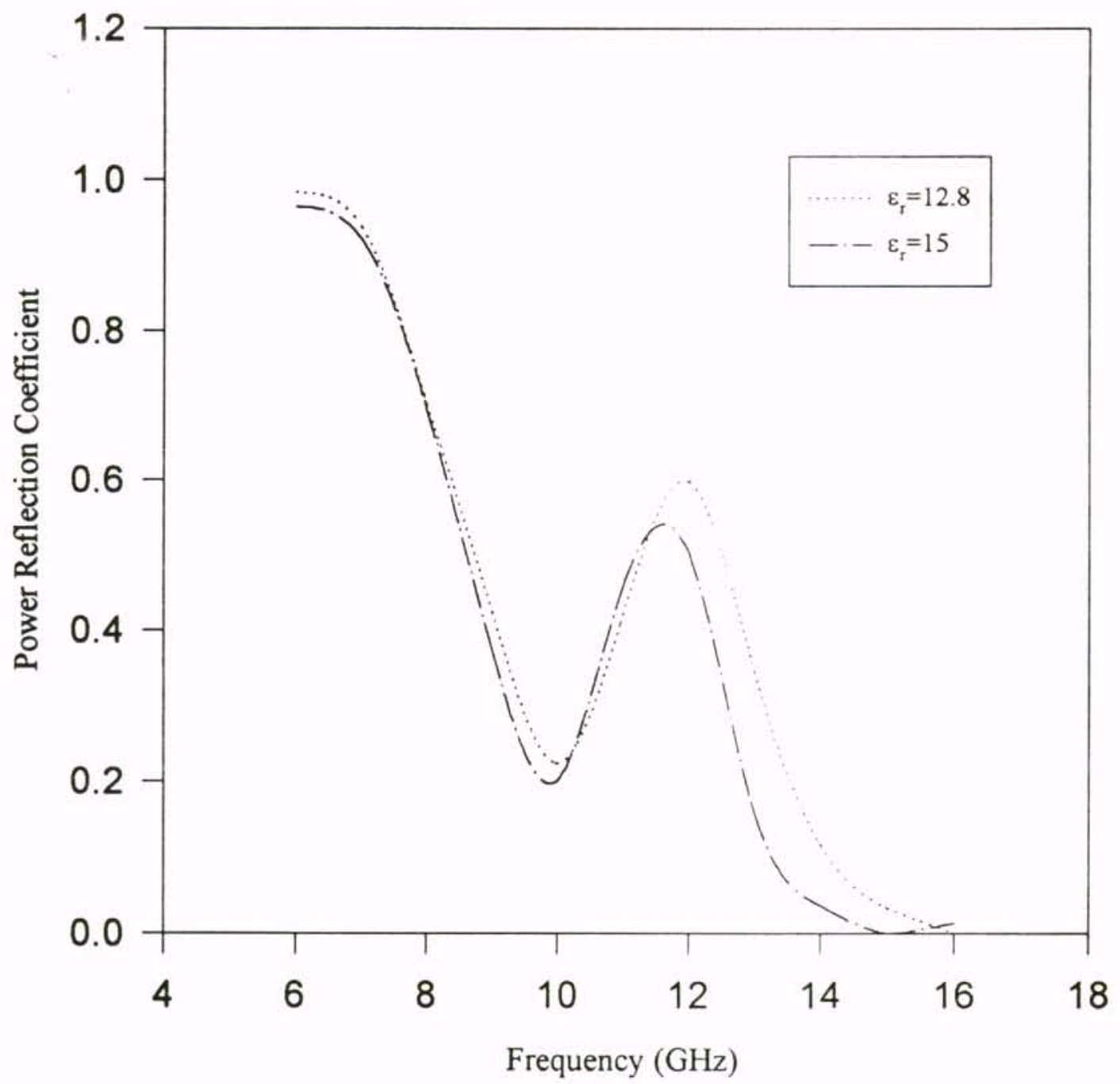

Figure 25. TE normal incidence, $y$-bias, $4 \pi \mathrm{M}_{\mathrm{s}}=1780 \mathrm{G}, \mathrm{d}=0.08 \mathrm{~cm}, \mathrm{H}_{0}=20000 \mathrm{e}$, with different dielectric constants $a \mathrm{a}=0.76 \mathrm{~cm}, \mathrm{bb}=0.254 \mathrm{~cm}, \mathrm{cc}=1.52 \mathrm{~cm}$, $\mathrm{dd}=1.35 \mathrm{~cm}$. 


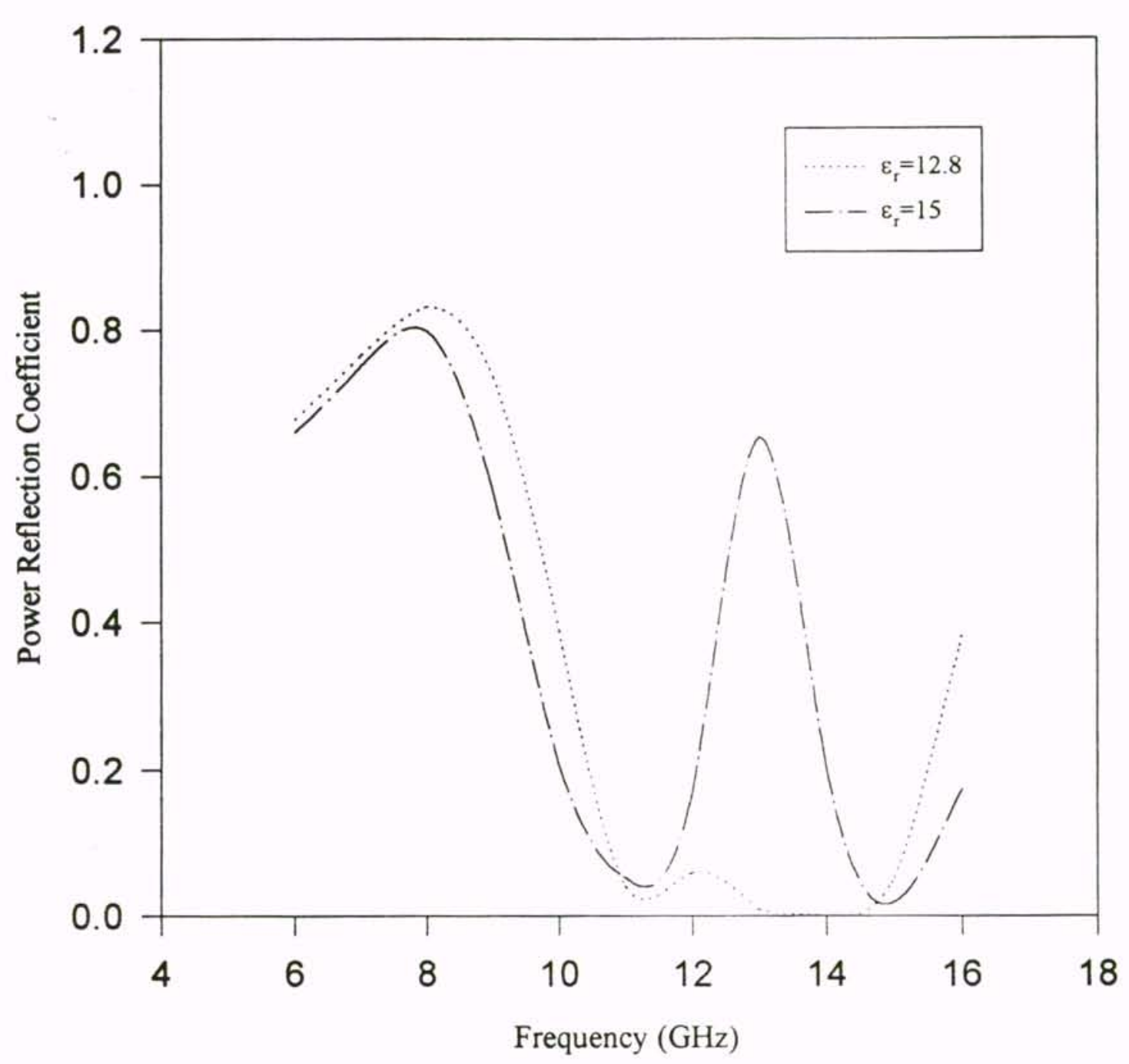

Figure 26. TE normal incidence, $y$-bias, $4 \pi \mathrm{M}_{\mathrm{s}}=1780 \mathrm{G}, \mathrm{d}=0.08 \mathrm{~cm}, \mathrm{H}_{0}=4000 \mathrm{Oe}$, with different dielectric constants. $a \mathrm{a}=0.76 \mathrm{~cm}, \mathrm{bb}=0.254 \mathrm{~cm}, \mathrm{cc}=1.52 \mathrm{~cm}$, $\mathrm{dd}=1.35 \mathrm{~cm}$. 


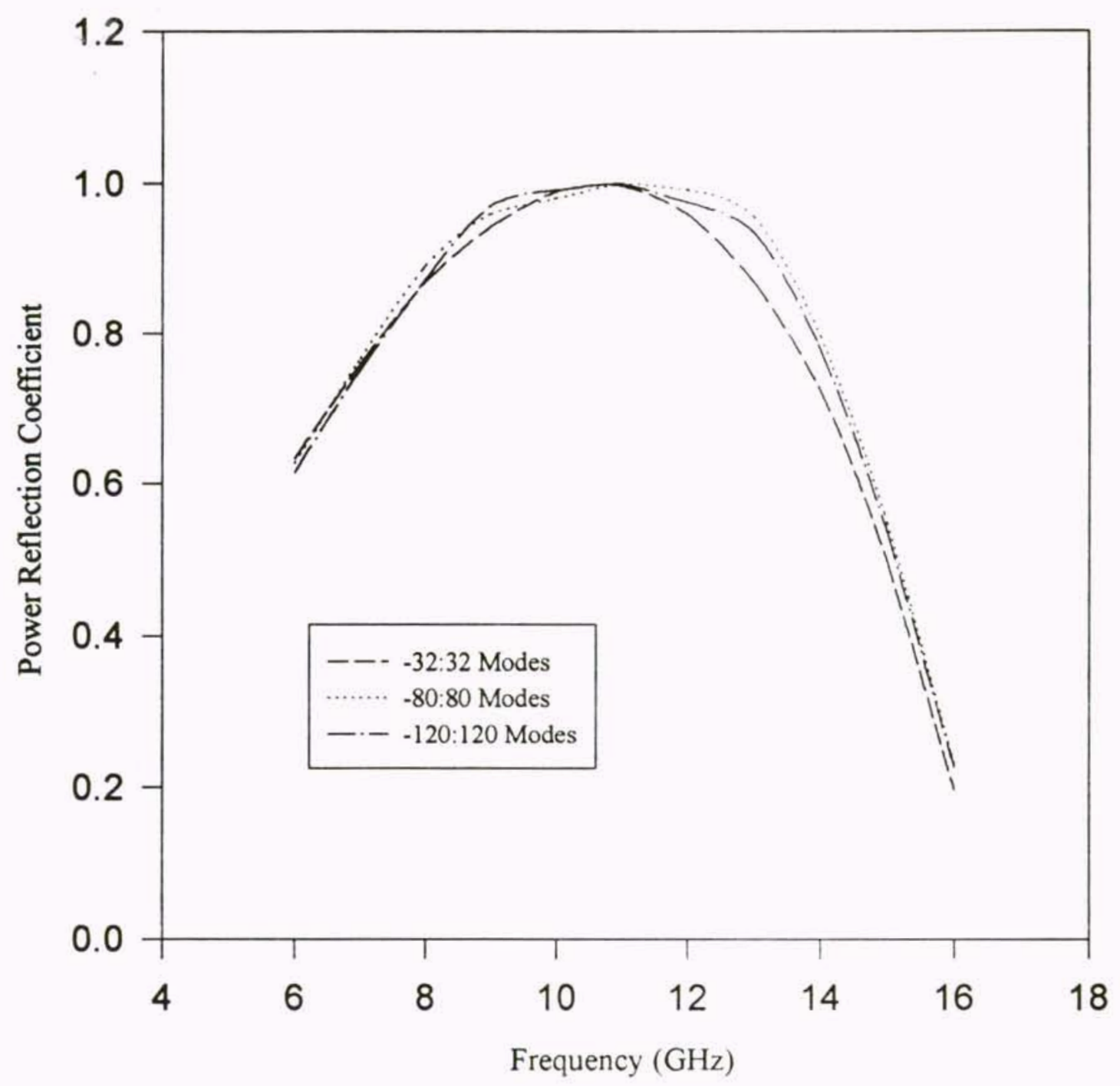

Figure 27. TE normal incidence, $y$-bias, $4 \pi \mathrm{M}_{\mathrm{s}}=17800 \mathrm{e}, \varepsilon_{\mathrm{r}}=12.8, \mathrm{~d}=0.02 \mathrm{~cm}$, $\mathrm{H}_{0}=50000$, with different calculating modes. $\mathrm{aa}=0.76 \mathrm{~cm}, \mathrm{bb}=0.254 \mathrm{~cm}$, $\mathrm{cc}=1.52 \mathrm{~cm}, \mathrm{dd}=1.35 \mathrm{~cm}$. 
results reported by T. K. Chang [41]. For the $\mathrm{z}$ direction bias cases in Figure 16, there is also such resonant frequency shifting.

Due to the permeability tensor of the ferrite, as well as the tedious matrices calculations in deriving the Green's function, the analysis becomes very complex. Thus, the change of the induced current on the patch calculated using the method of moments is abrupt in some frequency ranges. The second resonance also appears in its frequency response besides the main resonance. Such a phenomenon was also reported by T. K. Chang et al, in their experiments for other element shapes [41]. This characteristic is unique for frequency selective surfaces on ferrite substrates.

From Figures 17 to 22 , the influence of the ferrite substrate thickness to the resonant frequency is also apparent. The resonance decreases when the thickness of the substrate is increased. This is because that for such thickness level, the evanescent Floquet modes, decaying exponentially inside the substrate with distance, still have significant amplitudes, which in turn change the resonant frequency. This property is similar to that of the dielectric substrate [25].

The frequency selective surfaces on ferrite substrates seem not to be very sensitive to the dielectric constants of the ferrite. This can be seen from Figures 23 through 26.

The use of ferrite substrates also changes the shape of the bandpass response itself. Usually, the bandwidth of frequency selective surface on ferrite substrates is narrower than the free-standing and dielectric substrate cases. The main reason is that the permeability tensor changes as a function of the incident wave frequency. Some values 
of the frequency are in the vicinity of the singularities and an abrupt change of the induced current on the patch may be obtained. This causes the second resonance appearing, at the same time the frequency bandpass becomes narrower. 


\section{CHAPTER 6}

\section{DESIGN OF FREQUENCY SELECTIVE SURFACES USING}

\section{NEURAL NETWORK ALGORITHMS}

With the techniques discussed in the previous chapters, one can analyze the scattered fields as well as the reflection coefficient frequency response of the frequency selective surfaces with a set of given surface geometry parameters. But there is no closed form solution that relates a desired frequency response to the corresponding surface, directly. Therefore, to design a frequency selective surface for a given frequency response, one has to resort to a trial-and-error process. That is to say, one should assume a surface configuration (thus a set of parameters) and calculate its frequency response at first, then compare it with the desired one. This procedure is too laborious and timeconsuming. Here, an alternate design procedure, making use of neural network algorithms, is presented [42], [43]. The multilayer back-propagation algorithm [50], [51] along with an inversion algorithm [52] are used here.

The multilayer feedforward neural network is a network with at least one hidden layer between the input layer and the output layer. Every node is connected to the nodes in the adjacent layer by its weights. It is essentially an approximator; a mapping approximation from a finite dimensional input space to the output space. It belongs to 
the class of supervised learning. Such a neural network can be trained, with an iterative gradient descent algorithm designed to minimize the mean squared error between the desired target values and the actual output values, to learn from examples and to generalize from the training set to similar data not contained in the training examples. This process is called back-propagation algorithm. After the training, a set of weights for the network is obtained. The inversion of the neural network is done by computing iteratively an input vector which minimizes the Least-Mean-Square-Error to approximate a given output target. With this inversion algorithm, a lot of practical design problems can be solved.

The input of the multilayer feedforward network here are the geometric parameters of the frequency selective surfaces. The output is frequency response (or scattered field distribution) of the frequency selective surfaces. The general procedure for the design of frequency selective surfaces follows next.

First, the neural network is trained with the backpropagation algorithm. That is, one assumes a set of initial weights for the network arbitrarily, propagate the sample inputs forward from the input layer to the output layer. Next, one compares the propagated output data with sample output data, and a set of error signals is then obtained. The error signals are used to modify the weights by the gradient descent method. After the neural network is trained, a set of weights are obtained and kept unchanged. The mapping between the geometric parameters and the frequency response (or scattered field distribution) has thus been decided. For a practical application, a lot of sample patterns are needed. 
Next, the neural network is used to design frequency selective surfaces with the inversion algorithm. The gradient descent concept is used again here. For a given frequency response (or scattered field distribution), a set of initial neural network input data are chosen arbitrarily and propagated forward to the output layer. After comparing the output with the desired frequency response (or scattered field distribution), the obtained error signals are propagated downward to the input layer. The input data are modified by the input layer error signals. The modified input data is propagated forward again. With several such iterative steps, the output will converge to the desired frequency response (or scattered field distribution).

The neural network dynamics equations (defined in Appendix C) are:

$$
\begin{gathered}
h_{j}(1)=\sum_{1=1}^{N_{1-1}} W_{j i}(1) O_{i}(1-1)+\theta_{i}(1), \\
O_{j}(1)=\frac{1}{1+e^{-h_{j}(1)}}, \quad 1<j<N_{1}, 1<1<L,
\end{gathered}
$$

where $h_{j}(1)$ denotes the net input of $j$ th node at layer $1, O_{j}(1)$ denotes the activation of $j$ th node at layer $1 . \mathrm{L}$ is the number of layer (input layer is not included). $\theta_{i}(1)$ denotes the bias of node $i$ at layer 1 .

The neural network learning equation is given by:

$$
\Delta W_{j i}(1)=-\eta \frac{\partial E}{\partial W_{j i}(1)},
$$

where $\eta$ is the learning rate, error measure or cost function 


$$
E=\frac{1}{2} \sum_{j=1}^{N_{1}}\left[t_{j}-O_{j}(I)\right]^{2},
$$

$t_{j}$ is the sample pattern output for output layer node $j$.

Figure 28 gives the flow chart of back-propagation algorithm.

The equation for inversion of a neural network is:

$$
\Delta h_{j}(0)=-c \frac{\partial E}{\partial h_{j}(0)},
$$

where $c$ is the stepsize in input space.

If the input vector is denoted as I's, the inversion process iteratively leads to a sequence of input vectors

$$
I^{(0)}, I^{(1)}, I^{(2)}, \cdots \text {. }
$$

The inversion is completed if the distance between the output vector and the target $\mathrm{T}$ is small enough, i.e.

$$
\left[F\left(I^{(n)}\right)-T\right]^{2}<\epsilon,
$$

where $F($ ) denotes the mapping from input space to output space, $\varepsilon$ is the error criterion.

Figure 29 shows the flow chart of neural network inversion algorithm. Figure 30 is a unit cell of a (aperture type) frequency selective surface being modeled by the algorithms discussed above. The two input nodes of the neural network is assigned for the aperture dimension bb and dd. The other two aperture dimension parameters aa and cc remain unchanged. The scattered electric field distribution in the cell is chosen as the output of the network. There are totally 32 output nodes. The architecture of the neural 


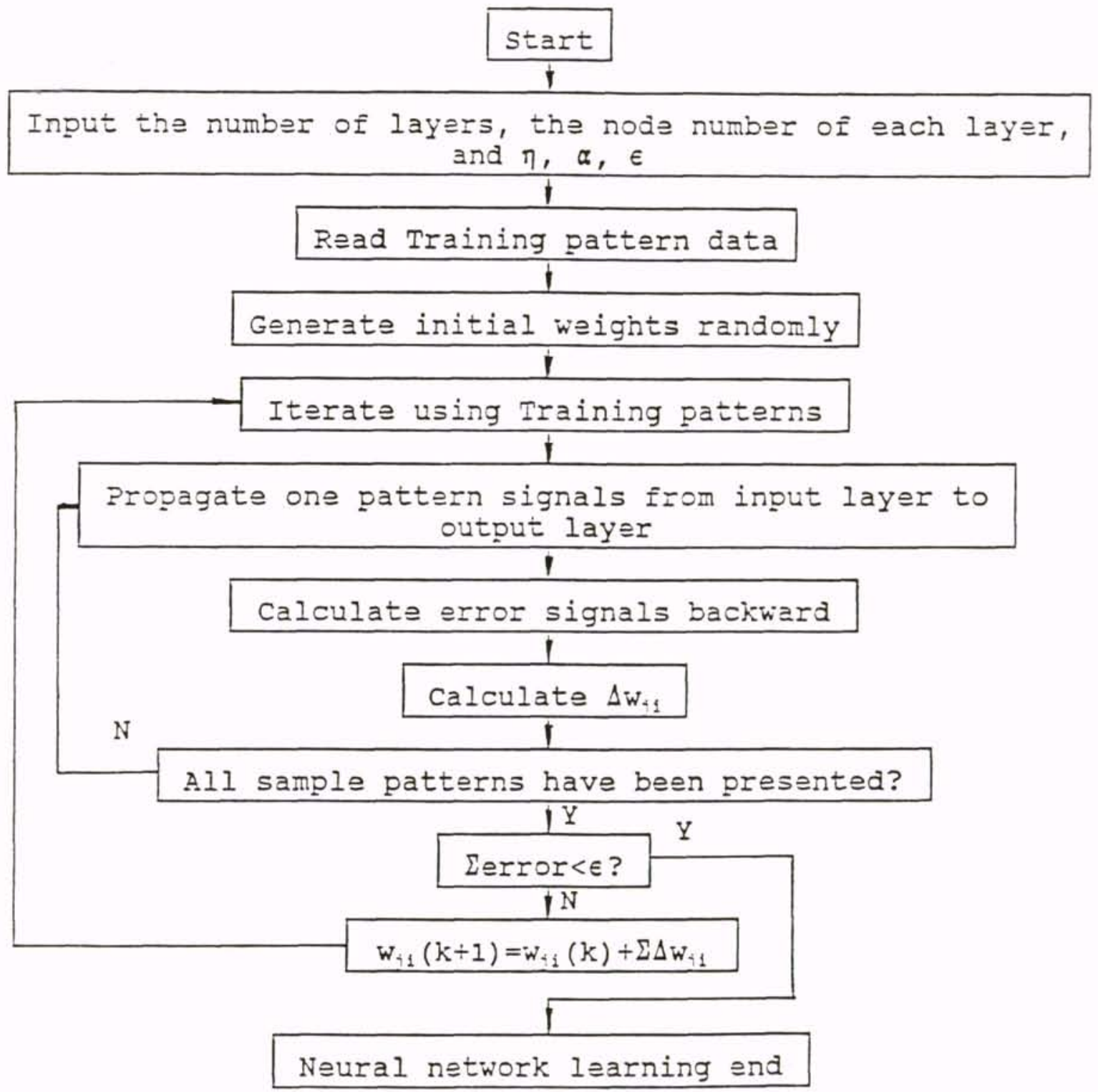

Figure 28. The flow chart of back-propagation algorithm. 


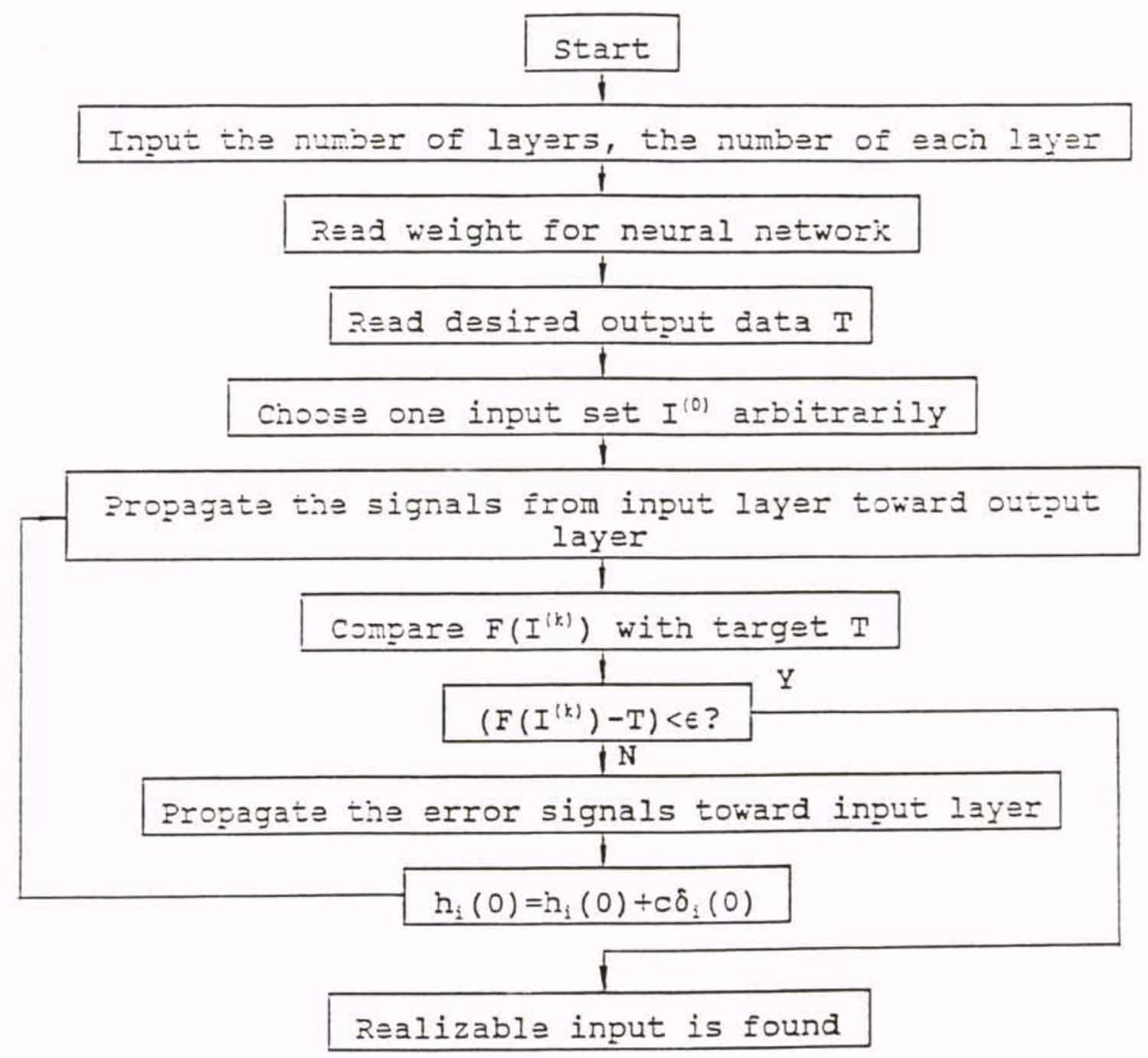

Figure 29. The flow chart of neural network inversion algorithm 


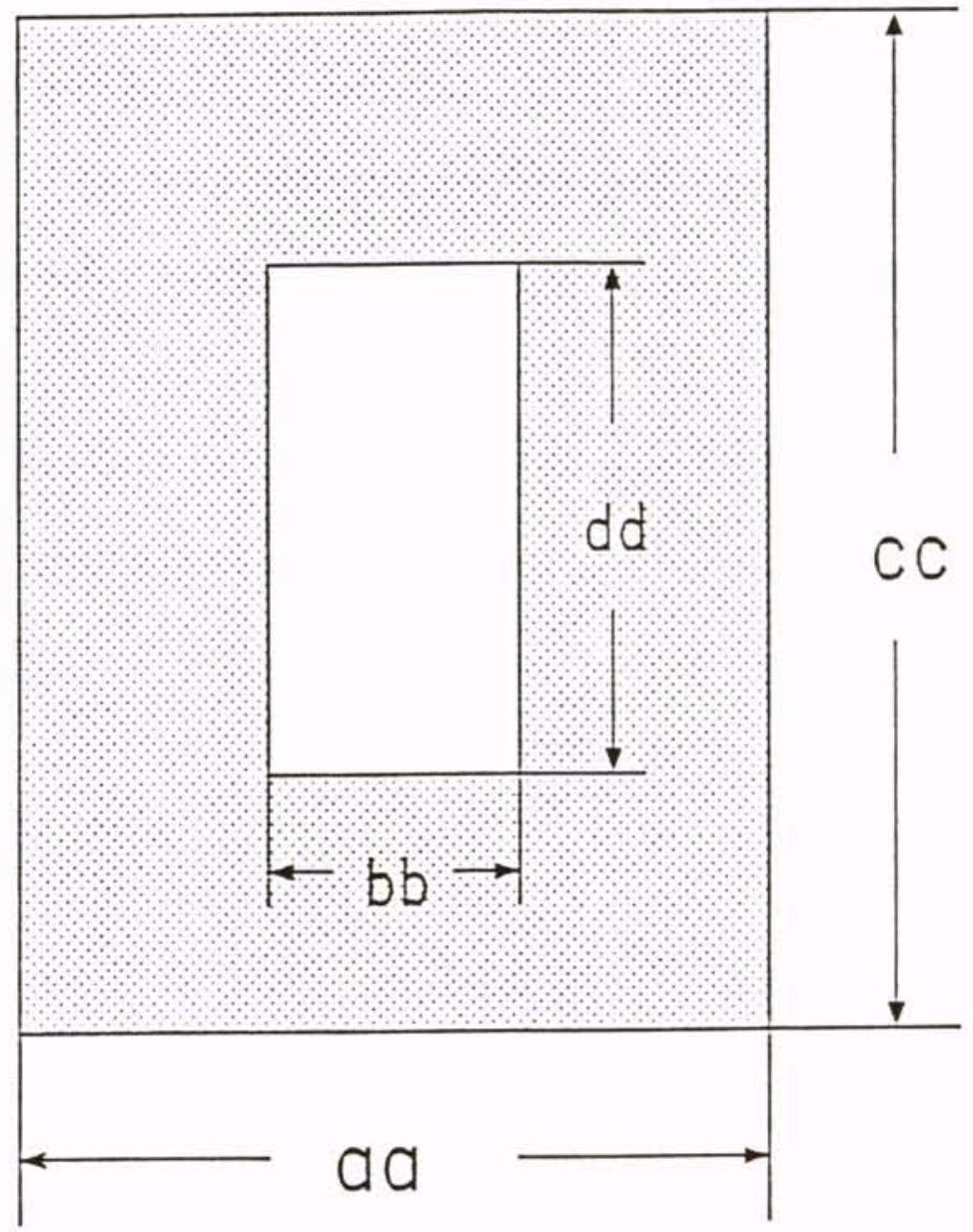

Figure 30. Geometry of a unit cell (aperture) for frequency selective surface. 
network used for the designing is a one hidden layer network, with 25 hidden nodes. The stepsize chosen here is usually 2 . The error criterion is 0.03 for both neural network training and inversion. 72 sample patterns are used to train the neural network.

Figures 31 through 34 are the design results. Here the "desired" ones mean the expected electric field distributions and the "designed" ones mean the results designed from neural network algorithms. It is apparent that the design results are quite satisfactory.

Although this work on neural network was done on free-standing case, it can be extended to the ferrite substrate case. 


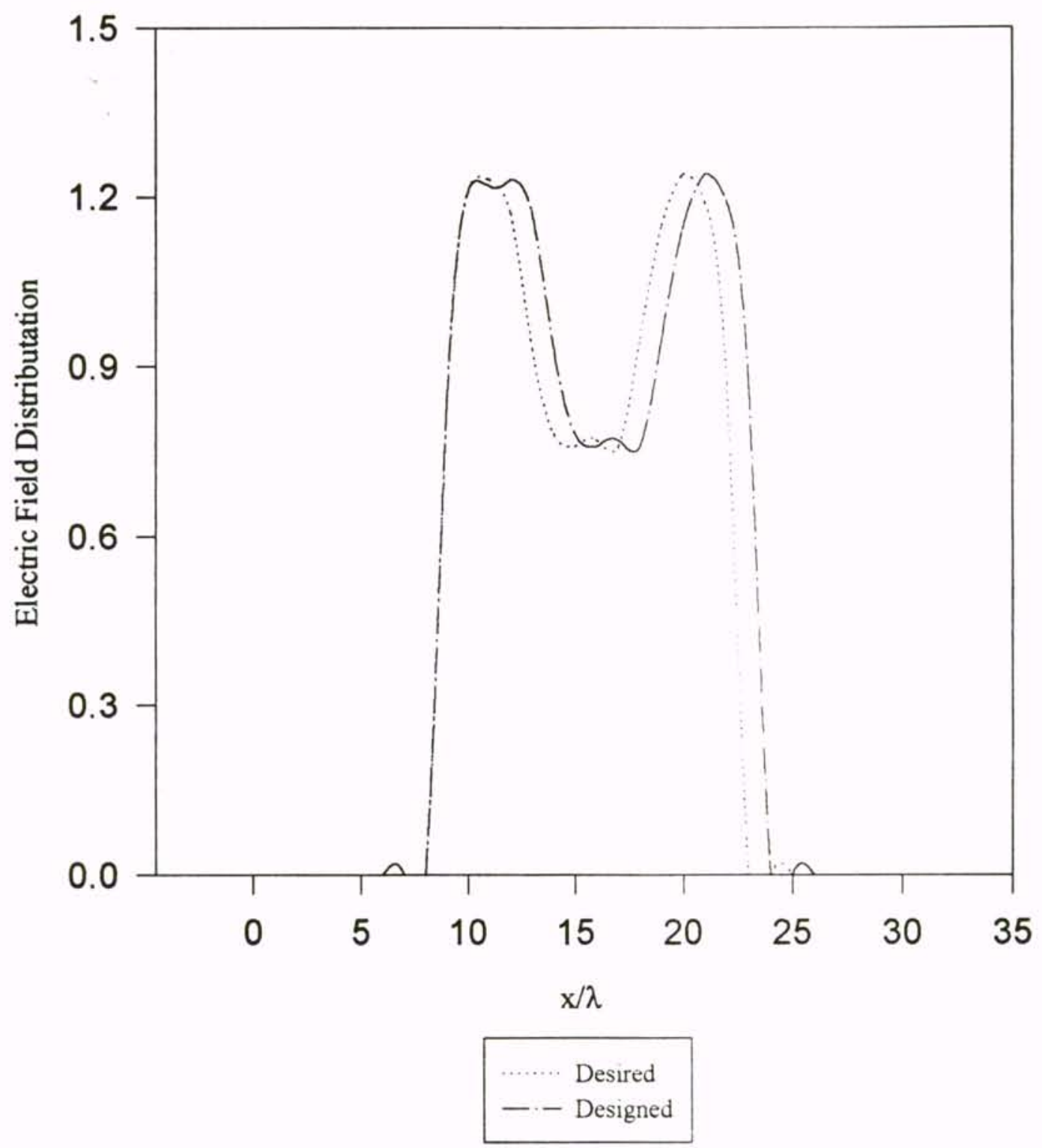

Figure 31. FSS designed by neural network algorithms, with aa $=1.0, \mathrm{cc}=1.0$, $b b=0.47, d d=0.44$. The parameters are divided by the wavelength. 


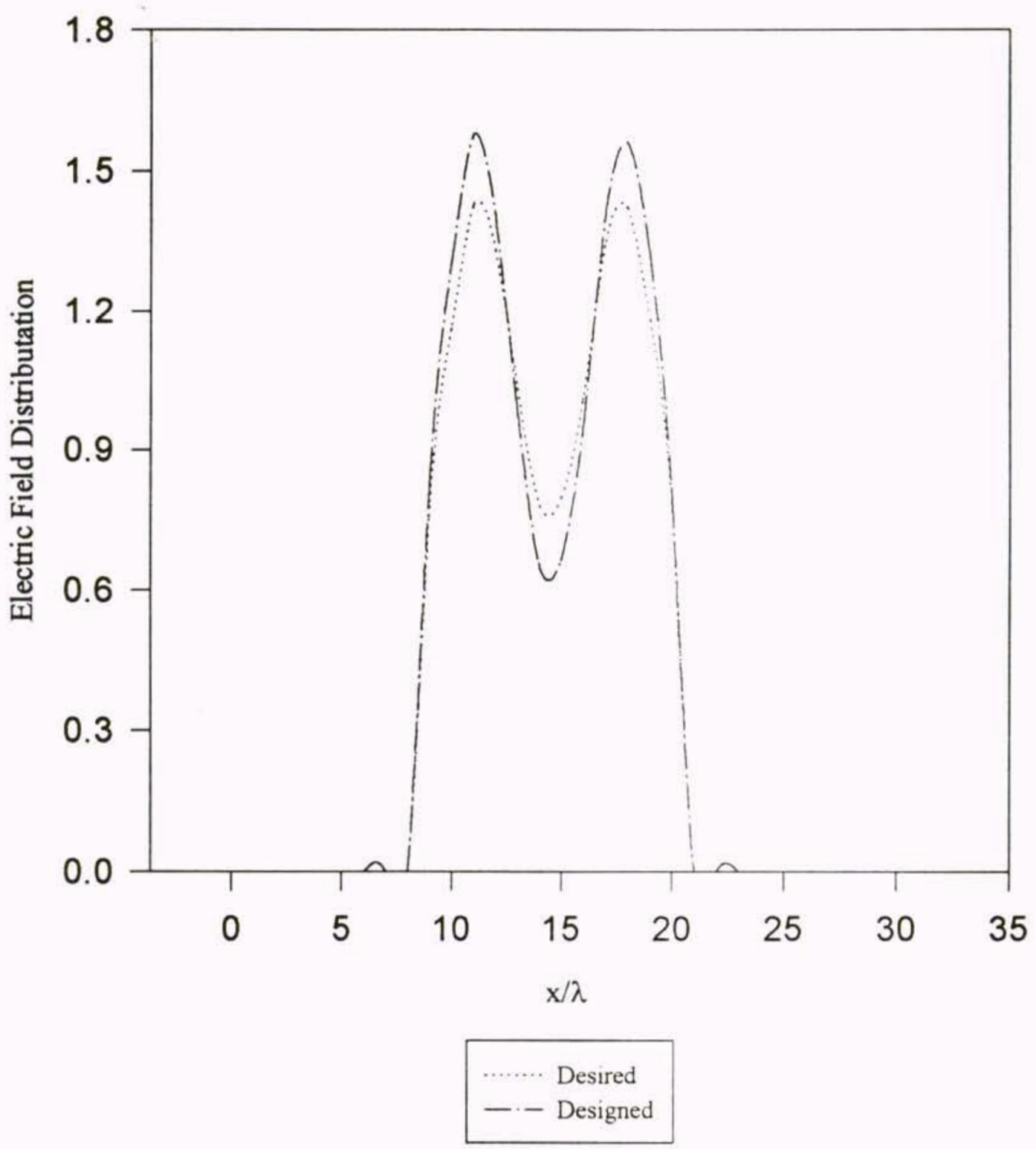

Figure 32. FSS designed by neural network algorithms, with $\mathrm{aa}=1.0, \mathrm{cc}=1.0$, $b b=0.40, d d=0.423$. The parameters are divided by the wavelength. 


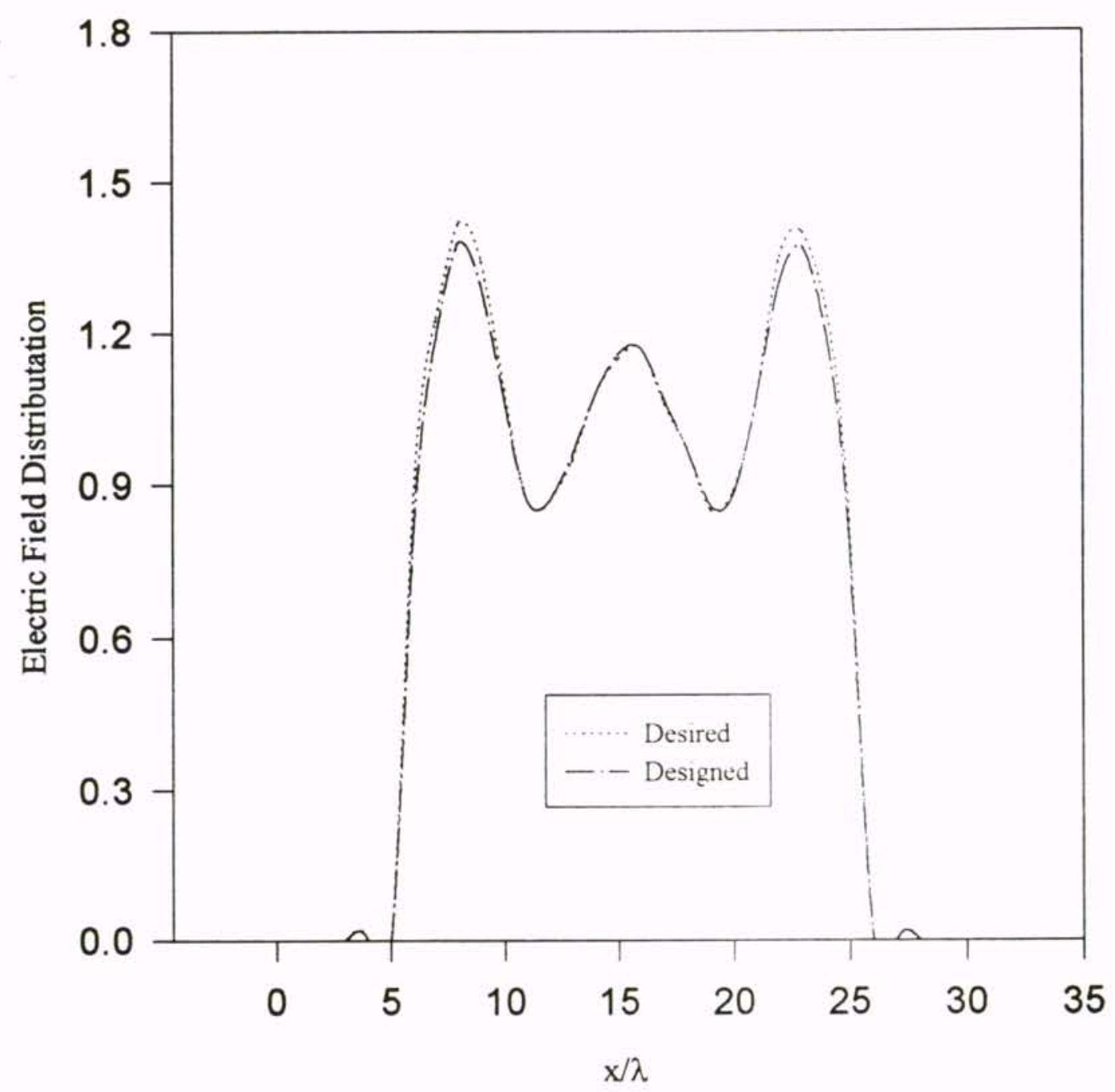

Figure 33. FSS designed by neural network algorithms, with aa $=1.0, \mathrm{cc}=1.0$, $b b=0.66, d d=0.65$. The parameters are divided by the wavelength. 


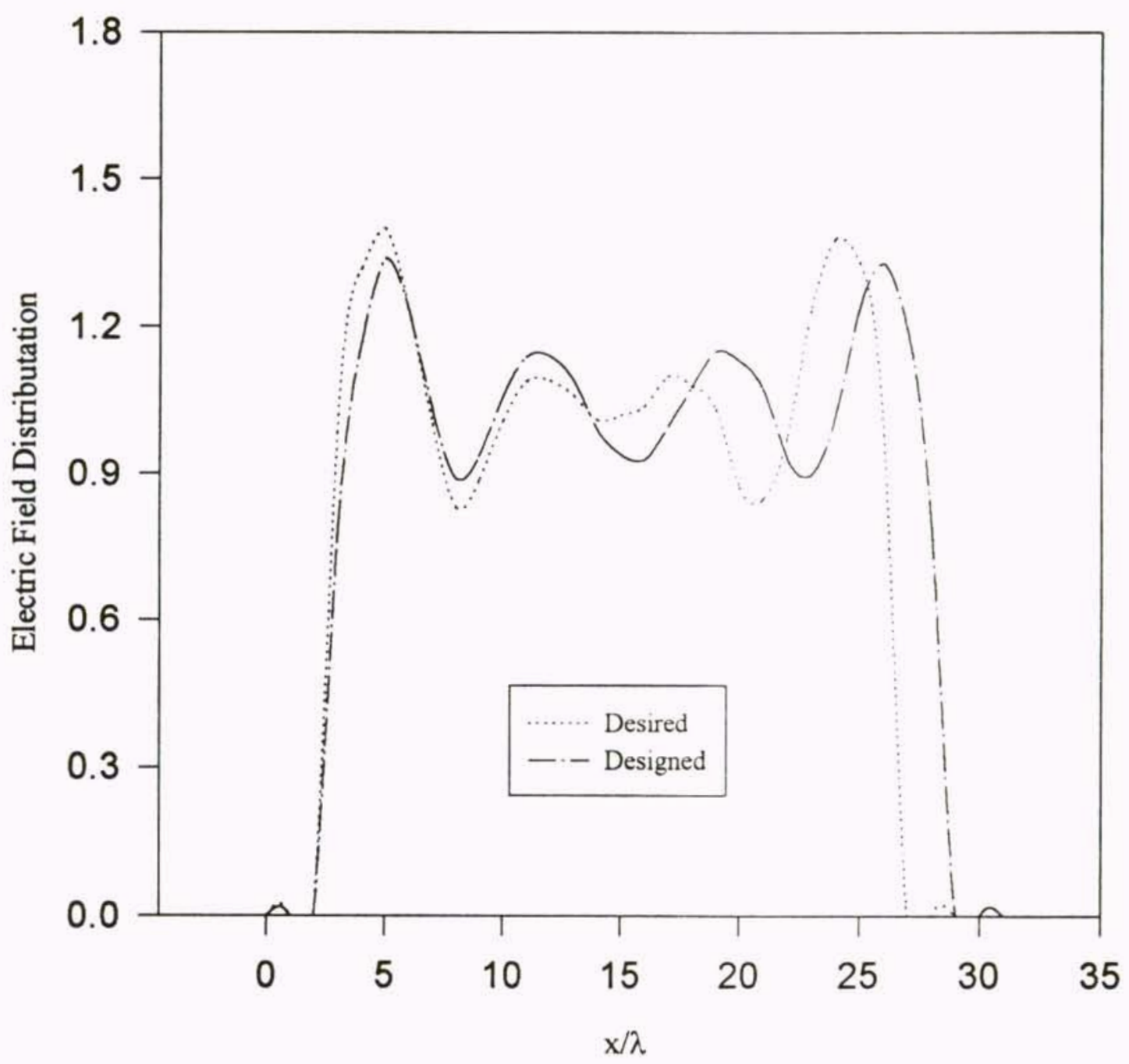

Figure 34. FSS designed by neural network algorithms, with aa=1.0, $\mathrm{cc}=1.0$, $\mathrm{bb}=0.83, \mathrm{dd}=0.65$. The parameters are divided by the wavelength. 


\section{CHAPTER 7}

\section{FUTURE WORK}

As discussed previously, the analysis of frequency selective surfaces on ferrite substrates is a very exciting topic which may find practical applications in microwave communication systems. Based on the theoretical model developed in the previous chapters, the following work can be done in the near future:

1. An investigation of the appearance of side lobes, along with the influence of the singularities of the model to the frequency response;

2. The use of different conductor patch configurations, such as cross-dipole, square loop, or skewed structures, to improve the performance of the frequency response;

3. The use of more than one layer of ferrite substrates for frequency selective surfaces or using the structure of the conductor patches being embedded in two ferrite slabs;

4. Combine of dielectric and ferrite substrates. 


\section{APPENDIX A \\ EXPRESSION OF GREEN'S FUNCTIONS FOR FSS \\ ON FERRITE SUBSTRATES}


Rewritting equation (181),

$$
\left[\begin{array}{c}
\tilde{E}_{x}^{B}\left(0_{+}\right) \\
\tilde{E}_{y}^{s}\left(0_{+}\right)
\end{array}\right]=\left[\begin{array}{ll}
\tilde{G}_{x x}\left(k_{x}, k_{y}\right) & \tilde{G}_{x y}\left(k_{x}, k_{y}\right) \\
\tilde{G}_{y x}\left(k_{x}, k_{y}\right) & \tilde{G}_{y y}\left(k_{x}, k_{y}\right)
\end{array}\right]\left[\begin{array}{c}
\tilde{J}_{x} \\
\tilde{J}_{y}
\end{array}\right],
$$

where

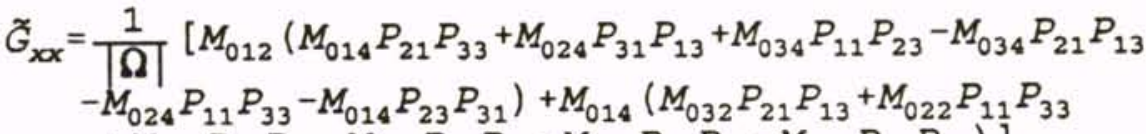

$$
\begin{aligned}
& \left.\left.+M_{012} P_{23} P_{31}-M_{012} P_{21} P_{33}-M_{022} P_{31} P_{13}-M_{032} P_{11} P_{23}\right)\right] \text {, } \\
& \begin{aligned}
& \tilde{G}_{x y}= \frac{1}{T \Omega}\left[M _ { 0 1 2 } \left(M_{014} P_{21} P_{43}+M_{024} P_{41} P_{13}+M_{044} P_{11} P_{23}-M_{044} P_{21} P_{13}\right.\right. \\
&\left.-M_{024} P_{11} P_{43}-M_{014} P_{23} P_{41}\right)+M_{014}\left(M_{042} P_{21} P_{13}+M_{022} P_{11} P_{43}\right. \\
&\left.\left.+M_{012} P_{23} P_{41}-M_{012} P_{21} P_{43}-M_{022} P_{41} P_{13}-M_{042} P_{11} P_{23}\right)\right]
\end{aligned} \\
& \begin{aligned}
\tilde{G}_{y x}= & \frac{1}{|\Omega|}\left[M _ { 0 2 2 } \left(M_{014} P_{21} P_{33}+M_{024} P_{31} P_{13}+M_{034} P_{11} P_{23}-M_{034} P_{21} P_{13}\right.\right. \\
& \left.-M_{024} P_{11} P_{33}-M_{014} P_{23} P_{31}\right)+M_{024}\left(M_{032} P_{21} P_{13}+M_{022} P_{11} P_{33}\right. \\
& \left.\left.+M_{012} P_{23} P_{31}-M_{012} P_{21} P_{33}-M_{022} P_{31} P_{13}-M_{032} P_{11} P_{23}\right)\right]
\end{aligned} \\
& \begin{aligned}
\tilde{G}_{y y}= & \frac{1}{|\Omega|}\left[M _ { 0 2 2 } \left(M_{014} P_{21} P_{43}+M_{024} P_{41} P_{13}+M_{044} P_{11} P_{23}-M_{044} P_{21} P_{13}\right.\right. \\
& \left.-M_{024} P_{11} P_{43}-M_{014} P_{23} P_{41}\right)+M_{024}\left(M_{042} P_{21} P_{13}+M_{022} P_{11} P_{43}\right. \\
& \left.\left.+M_{012} P_{23} P_{41}-M_{012} P_{21} P_{43}-M_{022} P_{41} P_{13}-M_{042} P_{11} P_{23}\right)\right]
\end{aligned}
\end{aligned}
$$

and

$$
\mathbf{Q}=\left[\begin{array}{cccc}
M_{012} & M_{014} & -P_{11} & -P_{13} \\
M_{022} & M_{024} & -P_{21} & -P_{23} \\
-M_{042} & -M_{044} & P_{41} & P_{43} \\
M_{032} & M_{034} & -P_{31} & -P_{33}
\end{array}\right],
$$




$$
\begin{array}{ll}
P_{j 1}=\sum_{i=1}^{4} T_{j i} M_{011} & j=1,2,3,4, \\
P_{j 3}=\sum_{i=1}^{4} T_{j i} M_{013} & j=1,2,3,4 .
\end{array}
$$

with

$$
\tilde{\mathbf{M}}_{0}=\left[\begin{array}{cccc}
\frac{k_{0}^{2}-k_{x}^{2}}{j \omega \epsilon_{0}} & \frac{k_{0}^{2}-k_{x}^{2}}{j \omega \epsilon_{0}} & 0 & 0 \\
-\frac{k_{x} k_{y}}{j \omega \epsilon_{0}} & -\frac{k_{x} k_{y}}{j \omega \epsilon_{0}} & -\lambda_{0} & \lambda_{0} \\
0 & 0 & \frac{k_{0}^{2}-k_{x}^{2}}{j \omega \mu_{0}} & \frac{k_{0}^{2}-k_{x}^{2}}{j \omega \mu_{0}} \\
\lambda_{0} & -\lambda_{0} & -\frac{k_{x} k_{y}}{j \omega \mu_{0}} & -\frac{k_{x} k_{y}}{j \omega \mu_{0}}
\end{array}\right]=\left[\begin{array}{cccc}
M_{011} & M_{012} & M_{013} & M_{014} \\
M_{021} & M_{022} & M_{023} & M_{024} \\
M_{031} & M_{032} & M_{033} & M_{034} \\
M_{041} & M_{042} & M_{043} & M_{044}
\end{array}\right],
$$

$$
\tilde{\boldsymbol{T}}=\left[\begin{array}{llll}
T_{11} & T_{12} & T_{13} & T_{14} \\
T_{21} & T_{22} & T_{23} & T_{24} \\
T_{31} & T_{32} & T_{33} & T_{34} \\
T_{41} & T_{42} & T_{43} & T_{44}
\end{array}\right] .
$$




\section{APPENDIX B}

\section{ELEMENT EXPRESSIONS FOR Z-BIAS CASE}


The detailed elements expressions for equation (190) are as follows

$$
\alpha_{1,2}=\frac{\mu k_{x} k_{y}-k_{x} k_{y}-j \kappa k_{e}^{2}+j \kappa k_{x}^{2}}{\lambda_{+}^{2}-\left(-j \kappa k_{x} k_{y}-\mu k_{\theta}^{2}+\mu k_{x}^{2}+k_{y}^{2}\right)},
$$

where $\alpha_{1}$ is equal to $\alpha_{2} \cdot \alpha_{3,4}$ have the same form as equation (220) with the exception of $\lambda_{+}$being replaced by $\lambda$.

$$
\beta_{1,2}= \pm \frac{1}{\omega \epsilon \lambda_{+}}\left[-\left(\kappa k_{\theta}^{2}+j k_{x} k_{y}\right) \alpha_{1,2}+\left(-j \mu k_{\theta}^{2}+j k_{x}^{2}\right)\right],
$$

where $\beta_{1}$ takes "+" sign, $\beta_{2}$ takes "-" sign. $\beta_{3,4}$ have the same form as equation (221) with the exceptions of $\lambda_{+}$being replaced by $\lambda_{-}$and $\alpha_{1,2}$ being replaced by $\alpha_{3,4}$

$$
\left.\gamma_{1,2}= \pm \frac{1}{\omega \epsilon \lambda_{+}}\left[j \mu k_{\theta}^{2}-j k_{y}^{2}\right) \alpha_{1,2}+\left(j k_{x} k_{y}-\kappa k_{\theta}^{2}\right)\right],
$$

where $\gamma_{1}$ takes "+" sign, $\gamma_{2}$ takes "-" sign. $\gamma_{3,4}$ have the same form as equation (222) with the exceptions of $\lambda_{+}$being replaced by $\lambda_{-}$and $\alpha_{1,2}$ being replaced by $\alpha_{3,4}$ 
APPENDIX C

DESCRIPTION OF NEURAL NETWORK ALGORITHMS USED 


\section{DERIVATION OF THE BACK-PROPAGATION ALGORITHM}

To simplify the notation, only one pattern is considered at first.

Cost function:

$$
E=\frac{1}{2} \sum_{j=1}^{N_{1}}\left[t_{j}-O_{j}(L)\right]^{2}
$$

Net input of node j:

$$
h_{j}(1)=\sum_{k=1}^{N_{l-1}} w_{j i}(1-1) o_{k}(1-1) \text {. }
$$

Activation of node $\mathrm{j}$ :

$$
O_{j}(1)=g\left[h_{j}(1)\right] \text {. }
$$

According to the gradient descent learning algorithm,

$$
\Delta w_{j i}(1-1)=-\eta \frac{\partial E}{\partial w_{j i}(1-1)}
$$

where $\frac{\partial E}{\partial w_{j i}(1-1)}$ can be written as the product of two parts using chain rule,

$$
\frac{\partial E}{\partial w_{j i}(1-1)}=\frac{\partial E}{\partial h_{j}(1)} \frac{\partial h_{j}(1)}{\partial w_{j 1}(1-1)} .
$$

Using equation (224),

$$
\frac{\partial h_{j}(1)}{\partial w_{j i}(1-1)}=O_{i}(1-1) \text {. }
$$


The other part of equation (227) can be written as

$$
\frac{\partial E}{\partial h_{j}(I)}=\frac{\partial E}{\partial o_{j}(I)} \frac{\partial o_{j}(1)}{\partial h_{j}(1)} .
$$

From equation (225),

$$
\frac{\partial o_{j}(l)}{\partial h_{j}(I)}=g^{\prime}\left[h_{j}(I)\right] .
$$

Then the expression of $\frac{\partial E}{\partial o_{j}(1)}$ needs to be derived. Two cases are considered.

Case $1 \quad 1=\mathrm{L}$

From (223)

$$
\frac{\partial E}{\partial o_{j}(I)}=-\left[t_{j}-o_{j}(L)\right] .
$$

Defining

$$
\delta_{i}(1)=-\frac{\partial E}{\partial h_{i}(1)},
$$

as the error at the layer 1 .

Combining equation (229), ( 230) and 231),

$$
\begin{aligned}
\frac{\partial E}{\partial h_{j}(1)} & =-\left[t_{j}-o_{j}(L)\right] g^{\prime}\left[h_{j}(L)\right] \\
& =-\delta_{j}(1)
\end{aligned}
$$

where 


$$
\delta_{j}(L)=g^{\prime}\left[h_{j}(L)\right]\left[t_{j}-O_{j}(L)\right],
$$

is the error of node $\mathrm{j}$ at output layer $\mathrm{L}$.

Substituting equation (228) and (233) into (227),

$$
\frac{\partial E}{\partial w_{j i}(1-1)}=-\delta_{j}(L) o_{j}(L-1)
$$

Inserting equation (226) to (235), yields

$$
\Delta w_{j i}(L-1)=\eta \delta_{j}(L) O_{j}(L-1) .
$$

Case $2 \mathrm{KL}$

Using chain rule to the first factor of equation (229),

$$
\begin{gathered}
\frac{\partial E}{\partial o_{j}(1)}=\sum_{k=1}^{N_{1+1}} \frac{\partial E}{\partial h_{k}(1+1)} \frac{\partial h_{k}(1+1)}{\partial o_{j}(1)} \\
=\sum_{k=1}^{N_{l+1}} \frac{\partial}{\partial o_{j}(1)} \sum_{m=1}^{N_{1}} w_{k m}(1) o_{m}(1) \\
=\sum_{k=1}^{N_{l+1}} \frac{\partial E}{\partial h_{k}(1+1)} w_{k j}(1) .
\end{gathered}
$$

From equation (233), $\frac{\partial E}{\partial h_{k}(1+1)}$ is nothing more than $\delta_{k}(1+1)$, then

$$
\frac{\partial E}{\partial o_{j}(1)}=-\sum_{k=1}^{N_{1+1}} \delta_{k}(1+1) w_{k j}(1) .
$$

Putting (238) back to equation (229) and using equation (230),

$$
\frac{\partial E}{\partial h_{j}(1)}=-g^{\prime}\left[h_{j}(1)\right] \sum_{k=1}^{N_{1 \cdot 1}} \delta_{k}(1+1) w_{k j}
$$


According to the definition of (233), equation (239) can be expressed as

$$
\frac{\partial E}{\partial h_{j}(1)}=-\delta_{j}(1)
$$

Combining equation (227) and (240),

$$
\frac{\partial E}{\partial w_{k j}(1-1)}=-\delta_{j}(1) o_{i}(1-1) .
$$

Putting equation (241) back to (226),

$$
\Delta w_{j i}(1-1)=\eta \delta_{j}(1) o_{i}(1-1)
$$

It is obvious that equation (236) and (242) have the same form. They follow the general delta rule. The error signals are backpropagated from output layer to input layer.

To update the weights,

$$
\Delta w_{j i}(I)=\sum_{u=1}^{p} \Delta w_{j i}^{u}(I),
$$

where $u$ is the number of sample patterns.

\section{INVERSION OF NEURAL NETWORK}

The cost function has the same definition as equation (223). After propagating the input data to the output layer, the error signals are propagated back to the input layer.

The error signals are defined as 


$$
\delta_{i}(1)=-\frac{\partial E}{\partial h_{i}(1)},
$$

where $\mathrm{l}=\mathrm{L}, \mathrm{L}-\mathrm{l}, \ldots, \mathrm{l}, 0$.

$\mathrm{l}=\mathrm{L}$

$$
\delta_{i}(L)=g^{\prime}\left[h_{i}(L)\right]\left[t_{i}-O_{i}(L)\right] .
$$

$\mathrm{l}<\mathrm{L}$

$$
\delta_{i}(1)=g^{\prime}\left[h_{i}(1)\right] \sum_{k=1}^{N_{l \cdot 1}} \delta_{k}(1+1) w_{k i}(1) .
$$

Using the gradient descent algorithm,

$$
\Delta h_{i}(0)=c \delta_{i}(0),
$$

where $\mathrm{c}$ is the stepsize. 


\section{REFERENCES}

[1] R. Mittra, C. H. Chan, and T. Cwik, "Techniques for Analyzing Frequency Selective Surfaces - A Review," Proceedings of the IEEE, vol. 76, No. 12, pp.1593-1615, December 1988.

[2] R. B. Kieburtz, and A. Ishimaru, "Aperture Fields of an Array of Rectangular Apertures," IEEE Trans. Antennas and Propagation, vol. AP-29, pp. 506-514, November 1961.

[3] C. C. Chen, "Scattering by a Two-Dimensional Periodic Array of Conducting Plates," IEEE Trans. Antennas and Propagation, vol. AP-18, pp. 660-665, September 1970.

[4] T. Itoh, "Spectral Domain Immitance Approach for Dispersion Characteristics of Generalized Printed Transmission Lines," IEEE Trans. Microwave Theory Techniques, vol. MTT-28, pp. 733-736, July 1980.

[5] Craig Scott, "Spectral Domain Method in Electromagnetics," Artech House, 1989.

[6] "Analysis Method for Eletromagnetic Wave problems," Chapter 11, Artech House, 1990.

[7] C. H. Tsao, and R. Mittra, "Spectral-Domain Analysis of Frequency Selective Surfaces Comprised of Periodic Arrays of Cross Dipoles and Jerusalem Crosses," IEEE Trans. Antennas and Propagation, vol. AP-32, pp. 478-486, May, 1984.

[8] C. H. Chan, and R. Mittra, "On the Analysis of Frequency-Selective Surfaces Using Subdomain Basis Function," IEEE Trans. Antennas and Propagation, vol. AP-38, pp. 40-50, January, 1990.

[9] R. F. Harrington, "Field Computation by Moment Methods," IEEE Press, 1993.

[10] B. J. Rubin, and H. L. Bertoni "Reflection from a Periodically Perforated Plane Using a Subsectional Current Approximation," IEEE Trans. Antennas and Propagation, vol. AP-31, pp. 829-836, November 1983.

[11] P. M. Van Den Berg, "Iterative Computational Techniques in Scattering Based upon the Integrated Square Error Criterion," IEEE Trans. Antennas and 
Propagation, vol. AP-32, pp. 1063-1070, October 1984.

[12] C. G. Christodoulou, Ph.D. Dissertation, North Carolina State University, Raleigh, NC, 1985.

[13] T. K. Sarkar, and E. Arvas, "On a Class of Finite Iterative Methods (Conjugate Directions) for the Solution of an Operator Equation Arising in Electromagnetics," IEEE Trans. Antennas and Propagation, vol. AP-33, pp. 10581066, October 1985.

[14] C. G. Christodoulou, and J. F. Kauffman, "On the Electromagnetic Scattering from Infinite Rectangular Grids with Finite Conductivity," IEEE Trans. Antennas and Propagation, vol. AP-34, pp. 144-154, February 1986.

[15] T. Cwik, and R. Mittra, "Scattering from a Periodic Array of Free-Standing Arbitrarily Sharped Perfectly Conducting or Resistive Patches," IEEE Trans. Antennas and Propagation, vol. Ap-35, pp. 1226-1234, November 1987.

[16] R. H. Ott, R. G. Kouyoumjian, and L. Peters, Jr., "Scattering by a TwoDimensional Periodic Array of Narrow Plates," Radio Science, vol. 2 (New Series), No. 11, pp. 1347-1359, November 1967.

[17] B. A. Munk, R. G. Kouyoumjian, and L. Peters, Jr., "Reflection Properties of Periodic Surfaces of Loaded Dipoles," IEEE Trans. Antennas and Propagation, vol. AP-19, pp. 612-617, September 1971.

[18] R. Mittra, R. C. Hall, and C. H. Tsao, "Spectral-Domain Analysis of Circular Patch Frequency Selective Surfaces," IEEE Trans. Antennas and Propagation, vol. AP-32, pp. 533-536, May 1984.

[19] S. W. Lee, "Scattering by Dielectric-Loaded Screen," IEEE Trans. Antennas and Propagation, vol. AP-19, pp. 656-665, September 1971.

[20] J. P. Montgomery, "Scattering by an Infinite Periodic Array of Thin Conductors on a Dielectric Sheet," IEEE Trans. Antennas and Propagation, vol. AP-23, pp. 70-75, January 1975.

[21] V. D. Agrawal, and W. A. Imbriale, "Design of a Cassegrain Subreflector," IEEE Trans. Antennas and Propagation, vol. AP-27, pp. 466-473, July 1979.

[22] C. H. Tsao, and R. Mittra, "A Spectral-Iteration Approach for Analyzing Scattering from Frequency Selective Surfaces," IEEE Trans. Antennas and Propagation, vol. AP-30, pp. 303-308, March 1982. 
[23] T. Cwik, R. Mittra, K. C. Lang, and T. K. Wu, "Frequency Selective Screens," IEEE Antennas and Propagation Society Newsletter, pp. 5-10, April 1987.

[24] H. L. Bertoni, L. H. S. Cheo, and T. Tamir, "Frequency-Selective Reflection and Transmission by a Periodic Dielectric Layer," IEEE Trans. Antennas and Propagation, vol. 37, pp. 78-83, January 1989.

[25] P. Callaghan, E. A. Parker, and R. J. Langley, "Influence of Supporting Dielectric Layers on the Transmission Properties of Frequency Selective Surfaces," IEE Proceedings-H, vol.138, No.5, pp. 448-454, October, 1991.

[26] S. W. Lee, G. Zarrillo, and C. L. Law, "Simple Formulas for Transmission Through Periodic Metal Grids or Plates," IEEE Trans. Antennas and Propagation, vol. AP-30, pp. 904-909, September 1982.

[27] R. C. Hall, R. Mittra, and K. M. Mitzner, "Analysis of Multilayered Periodic Structures Using Generalized Scattering Matrix Theory," IEEE Trans. Antennas and Propagation, vol. 36, pp. 511-517, April 1988.

[28] T. Cwik, and R. Mittra, "The Cascade Connection of Planar Periodic Surfaces and Lossy Dielectric Layers to Form an Arbitrary Periodic Screen," IEEE Trans. Antennas and Propagation, vol. AP-35, pp. 1397-1405, December 1987.

[29] C. Christodoulou, D. P. Kwan, R. Middleveen, and P. F. Wahid, "Scattering from Stacked Gratings and Dielectrics for Various Angles of Wave Incidence," IEEE Trans. Antennas and Propagation, vol. 36, pp. 1435-1442, October 1988.

[30] C. K. Lee, and R. J. Langley, "Equivalent-circuit Models for Frequency-selective Surfaces at Oblique Angles of Incidence," IEE Proceedings vol. 132, Pt. H, No.6, pp. 395-399, October 1985.

[31] C. H. Chan, "A Numerically Efficient Technique for the Method Moments Solution of Electromagnetic Problem Associated with Planar Periodic Structures," Microwave And Optical Technology Letters, vol. 1 No. 10, pp. 372-374, December 1988.

[32] T. K. Wu, "Single-Screen Triband FSS with Double-Square-Loop Elements," Microwave And Optical Technology Letters, vol. 5, No. 2, pp.56-59, February 1992.

[33] W. L. Ko, and R. Mittra, "Scattering from Array of Metallic Patches Located on a Curved Surface," IEEE Antennas and Propagation Society International Symposium, pp. 642-645, Syracuse, NY, June 1988. 
[34] P. W. Grounds, and K. J. Webb, "Numerical Analysis of Finite Frequency Selective Surfaces with Rectangular Patches of Various Aspect Ratios," IEEE Trans. Antennas and Propagation, vol. 39, pp. 569-575, May 1991.

[35] H. L. Glass, "Ferrite Films for Microwave and Millimeterwave Devices," Proceedings Of The IEEE, vol. 76, No.2, pp. 151-158, February 1988.

[36] El-Badawy El-Sharawy, and R. W. Jackson, "Coplanar Waveguide and Slot Line on Magnetic Substrates: Analysis and Experiment," IEEE Trans. Microwave Theory and Techniques, vol. 36, pp. 1071-1079, June 1988.

[37] D. M. Pozar, "Radiation and Scattering Characteristics of Microstrip Antennas on Normally Biased Ferrite Substrates," IEEE Trans. Antennas and Propagation, vol. 40, pp. 1084-1092, September 1992.

[38] N. E. Buris, T. B. Funk, and R. S. Silverstein, "Dipole Arrays Printed on Ferrite Substrates," IEEE Trans on Antennas and Propagation, vol. 41, pp. 165-176, February 1993.

[39] Y. Liu, C. G. Christodoulou, P. F. Wahid, and N. E. Buris, "Analysis of Frequency Selective Surfaces with Ferrite Substrates," IEEE Antennas and Propagation Society International Symposium, pp. 1640-1643, Newport Beach, CA, June 1995.

[40] G. Y. Li, Y. C. Chan, T. S. Mok, and J. C. Vardaxoglou, "Analysis of Frequency Selective Surfaces on Biased Ferrite Substrate," IEEE Antennas and Propagation Society International Symposium, pp. 1636-1639, Newport Beach, CA, June 1995.

[41] T. K. Chang, R. J. Langley, and E. A. Parker, "Frequency Selective Surfaces on Biased Ferrite Substrates," Electronics Letters, pp. 387-388, 1994, 30.

[42] J N. Hwang, C. H. Chan, and R. J. Marks II, "Frequency Selective Surface Design Based on Iterative Inversion of Neural Networks," Proc. International Joint Conference on Neural Networks, pp. I34-I44, San Diego, CA, June 1990.

[43] D. T. Davis, C. H. Chan, and J. N. Hwang, "Frequency Selective Surface Design Using Neural Networks Inversion Based on Parametrized Representations," IEEE Antennas and Propagation Society International Symposium, pp. 200-203, The University of Western Ontario, Canada, 1991.

[44] R. F. Harrington, "Time-Harmonic Electromagnetic Fields," McGraw-Hill, 1961. 
[45] C. A. Balanis, "Advanced Engineering Electromagnetics," John Wiley \& Sons, 1989.

[46] R. E. Collin, "Foundations for Microwave Engineering," McGraw-Hill, 1992.

[47] S. Ramo, J. R. Whinnery, and T. V. Duzer, "Fields and Waves in Communication Electronics," John Wiley \& Sons, 1984.

[48] D. M. Pozar, "Microwave Engineering," Addison-Wesley, 1990.

[49] Hung-Yu Yang, "A Numerical MEthod of Evaluating Electromagnetic Fields in a Generalized Anisotropic Medium," IEEE Trans. Microwave Theory Theory and Techniques, Vol. 43, No. 7, pp 1626-1628, July, 1995.

[50] D. E. Rumelhert, G. E. Hinton, and R. J. Williams, "Learning Internal Representations by Error Propagation. Parallel Distributed Processing (PDP): Exploration in the Microstructure of Cognition (Vol. 1), Chapter 8, MIT Press, Massachusetts, 1986.

[51] John Hertz, Anders Krogh, and Richard G. Palmers, "Introduction to the Theory of Neural Computation," Addison-Wesley, 1991.

[52] A. Linded, and J. Kinderman, "Inversion of Multilayer Nets," Proc. Int'l Joint Conf. on Neural Networks, Washington D. C., pp. 425-430, June 1989. 


\section{VITA}

Youcheng Liu was born in Shanghai, China in 1959. He received the Bachelor of Science degree and the Master of Science degree in Electrical Engineering from Shanghai Jiao-Tong University in 1982 and 1990, respectively. From 1982 to 1991, he worked at Shanghai Institute of Electric Power, as an instructor. In 1991, he came to United States and started his Ph.D. study at Electrical and Computer Engineering Department, University of Central Florida. During his Ph.D. studies, he was first a graduate teaching assistant and then a graduate research assistant at Electrical and Computer Engineering Department, University of Central Florida. He will receive the Doctor of Philosophy degree in May 1996.

Mr. Liu has a broad interest in electrical engineering areas. His research interests include numerical techniques in electromagnetics, frequency selective surfaces, neural network algorithms, microwave circuits, and application software programming. 


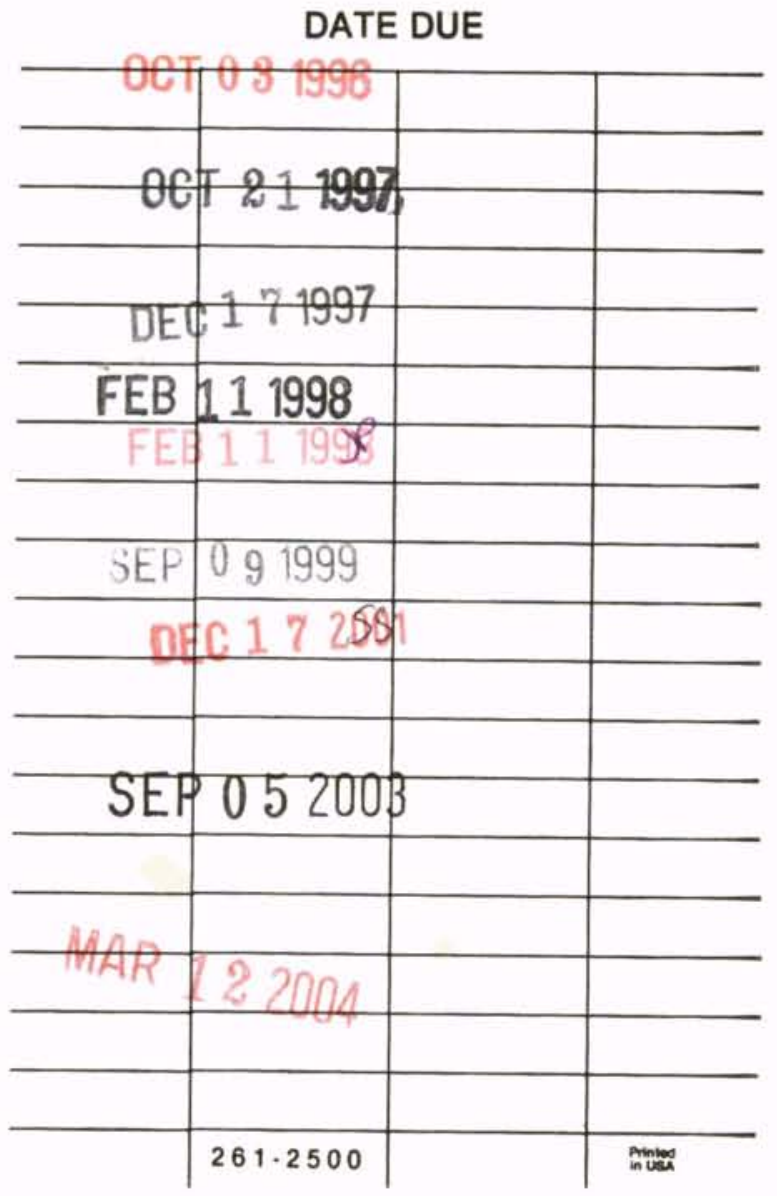


